\title{
Dynamic Pricing Game in a Dual-channel Closed- loop Supply Chain with Heterogeneous Players and Delay Decision
}

\section{Yuhao Zhang}

Shanghai University of Finance and Economics

Tao Zhang ( $\nabla$ taozhang@mail.shufe.edu.cn )

Shanghai University of Finance and Economics

\section{Research Article}

Keywords: Dual-channel CLSC, pricing, Bounded rationality, Delay decision, Stackelberg game, Bifurcation

Posted Date: April 30th, 2021

DOl: https://doi.org/10.21203/rs.3.rs-430480/v1

License: (c) (1) This work is licensed under a Creative Commons Attribution 4.0 International License.

Read Full License 


\title{
Dynamic pricing game in a dual-channel closed-loop supply chain with heterogeneous players and delay decision
}

\author{
Yuhao Zhang ${ }^{\text {a }}$, Tao Zhang ${ }^{\text {a, b }}$ \\ a School of Information Management and Engineering, Shanghai University of Finance and \\ Economics, Shanghai, 200433, China \\ b Shanghai Key Laboratory of Financial Information Technology (Shanghai University of Finance \\ and Economics), Shanghai, 200433, China \\ Correspondence should be addressed to Tao Zhang, taozhang@mail.shufe.edu.cn
}

\begin{abstract}
In this paper, we study a dual-channel closed-loop supply chain(CLSC), where the manufacturer wholesales the new product through the traditional retail channel and distributes the remanufactured product via a direct channel established by himself. We focus on developing two dynamic Stackelberg game models under the assumption of the retailer is an adaptive agent and the manufacturer is a bounded rational player with non-delay and delay decisions. The existence and locally asymptotic stability of Nash equilibrium is investigated, and also the complex dynamics of each model is illustrated including period-doubling bifurcation, Neimark-Sacker bifurcation, strange attractor and chaotic phenomena. Numerical simulations are conducted to examine the impacts of key parameters on the complex behaviors of the long-run dynamic Stackelberg game and the performance of chain members under various scenarios. The results reveal that the excessively high value of the price adjustment speed of the manufacturer, the consumer discount perception for the remanufactured product as well as the consumer preference degree to the direct channel have a destabilization effect on the Nash equilibrium. Besides, the delay decision adopted by manufacturer no matter in the traditional or direct channel does not always necessarily make the system more stable, but the appropriately delay weights can expand the stability domain of the system. Moreover, the manufacturer would suffer a significant profit loss while the retailer can capture more profits when the dual-channel CLSC system falls into periodic cycles and chaos motions. At last, the variable feedback control method is utilized to eliminate the delayed system chaos.
\end{abstract}


Keywords: Dual-channel CLSC; pricing; Bounded rationality; Delay decision; Stackelberg game; Bifurcation

\section{Introduction}

Due to the heavy environmental pollution and resource shortage all around the world, many countries, such as the US, Japan, China, and so on have issued the stringent environment legislations and take-back regulations to require the manufacturer to reused their end-of-life products [1]. In recent years, remanufacturing is considered as an effective method to achieve environmentally sustainable development and create additional economical values, which have been recognized both in academia and practitioners [2]. Accordingly, a lot of leading companies, such as Apple, Dell, HP, and Xerox, etc., have incorporated the remanufacturing process into their reverse supply chain for undertaking social responsibility and reducing production cost.

Remanufacturing is a process in which used products are disassembled and its parts are repaired and used in the production of new products [3]. When both the regular production and remanufacturing are carried out by a manufacturer, then the supply chain management associated will become more complicated. Consequently, some questions will be incurred when a manufacturer sells the new product and remanufactured product simultaneously, such as the choice of distribution channel structure, pricing strategies between new and remanufactured products as well as collection channel selection. Even though those research questions have received increasing attention from scholars but the related research quite limited. To this end, our work tries to deal with the research questions aforementioned in a dual-channel CLSC framework.

A great number of scholars have discussed the optimal price and collection channel selection in the CLSC [4-6]. Choi et al. [7] examined the optimal decisions on pricing and performance of different CLSCs under different channel leadership. Modak et al. [8] analyzed the effects of recycling and product quality level on pricing decisions in a two-echelon CLSC under the Stackelberg game setting. Jalali et al. [9] investigated the best configuration for a CLSC dealing with the complementary goods. The work pointed out that the presence of complementary products makes the CLSC selection extremely difficult and entails several trade-offs among collection performance, prices, and profits. However, most of the aforementioned literature only considers selling the new and remanufactured products in a single channel with the same price. 
In addition, there exists a branch of literature has researched the pricing strategies in the dualrecycling channel CLSC context [10-12]. Huang et al. [13] examined the optimal strategies of price and return rate in a CLSC with dual recycling channel by considering the competition intensity between recycling channels. The result showed that the CLSC with a dual-recycling channel outperforms the CLSC with a single recycling channel from the perspectives of the manufacturer and the consumers. Ranjbar et al. [14] evaluated optimal pricing and collection decisions with the retailer and third-party dual collection channel under different channel leadership in a three-level CLSC. Although the abovementioned articles have widely discussed the pricing decisions under different frameworks in CLSC; however, those works ignoring the heterogeneous characteristics of the consumers for the remanufactured product. In contrast to the paper abovementioned, our paper proposes a dynamic Stackelberg game model in which new and remanufactured products are clearly distinguishable for the consumers, and also differentiated price is advocated to execute with separate channels in a dual-channel CLSC.

In reality, assuming that the new and remanufactured products are indistinguishable is not applicable in most cases. Due to the remanufactured products are usually perceived as lower quality compared with the new ones, so the customer's willingness-to-pay for new and remanufactured products will be likely different [15]. Some studies have addressed differentiation between new and remanufactured products. Ferrer and Swaminathan [16] analyzed the optimal remanufacturing and pricing strategy for the game in two-period, multi-period and infinite planning horizons by assuming the remanufactured product is differentiated from the new product. Wu [17] considered a supply chain consisting of one manufacturer, one remanufacturer and one retailer. Both manufacturers bundle their products with services. The analyses revealed that when remanufacturing leads to more cost-savings, the remanufacturer will provide a higher service level to the customers than the traditional manufacturer. Chen and Chang [18] developed the analytical models using Lagrangean relaxation and dynamic programming schemes to deal with managing two differentiated versions of the same product. However, the literature abovementioned does not study that the important role of direct channel which added by manufacturer in the dual-channel CLSC.

The rapid development of E-commerce and Internet technology has been prompting many prominent companies to establish their own direct channels for capturing more market share and retaining more loyal customers, such as IBM, HP, Apple and Cisco [19]. There is a substantial body 
of literature has explored the optimal price decisions and performance of chain members in the dualchannel supply chain context. Chiang et al. [20] argued that the strategic use of the direct marketing can mitigate the double marginalization. Tsay and Agrawal [21] indicated that adding a direct channel alongside a reseller channel by the manufacturer is not necessarily detrimental to the reseller. On the contrary, both parties will better off in some cases. Besides, this work also examined how to adjust the manufacturer-retailer relationship. Yan et al. [22] investigated a dual-channel supply chain use the game theory approach by considering the consumer acceptance degree to the direct channel. The result revealed that both the manufacturer and the retailer always benefit from a dual-channel profit-sharing because of the incremental profit gains generated by the dual-channel strategy. The optimal pricing decisions and performance of channel members in a dual-channel supply chain with considering retailer's service efforts are analyzed in [23]. The paper employed above only focuses on the pricing decisions in the forward dual-channel supply chain but without considering remanufacturing.

The research about dual-channel CLSC has been widely recognized in the literature. An increasing number of authors have addressed the questions related to pricing, channel coordination and channel power structure in the dual-channel CLSC under the assumption of the consumers' perception of the new and remanufactured products is identical. Kong et al. [24] explored the optimal pricing and service decision in a dual-channel CLSC where the manufacturer is responsible for remanufacturing. Xie et al. [25] introduced a Stackelberg game model via combining the revenue-sharing contract in the forward channel with the channel investment cost-sharing contract in a dual-channel CLSC. Zheng et al. [26] examined the effects of the forward channel competition and different power structures on dual-channel CLSC. The optimal price policies and performance of channel members are analyzed in detail. The result found that each channel member has an incentive to play the channel leader's role. However, those works do not involve the problem of channel structure selection for new and remanufactured products in the presence of heterogeneous consumers who evaluate two types of products differently.

The potential for the cannibalization of new product sales by remanufactured products is a central issue in CLSC. In practice, many manufacturers are concern about the remanufacturing might cannibalize the new product's sale [27-28]. In order to alleviate the cannibalization between new and remanufactured products. Some giant companies wholesale their new and remanufactured 
products through two separate channels in dual-channel CLSC. For instance, Apple and Canon sell their remanufactured products via their own online official stores [29]. Similarly, Dell and HP sell their remanufactured computers in their online outlet stores [30-31]. Atasu et al. [32] believed that the remanufacturing does not always cannibalize new product sales by using the proper pricing strategies as well as pointed out that the pricing strategies and competition between new and remanufactured products is not a static decision. Therefore, it is necessary and essential for the model to implement the price differentiation for two types of products with separate distribution channels.

Several studies have been done on analyzing the interactions between the chain members in the dual-channel CLSC in which the manufacturer distributes the new product in the traditional channel and sells the remanufactured product through the direct channel. The relevant research has demonstrated that this channel structure could mitigate the cannibalization between new and remanufactured products [33]. Jiang et al. [34] investigated the pricing strategy related to such channel structure using agent technologies for analyzing the system. The work found that the optimal profits are increased by increasing direct channel and remanufacturing. Gan et al. [35] developed a pricing decision model for short life-cycle products in such dual-channel CLSC. Besides, the two scaling factors, that is, the consumer acceptance for the remanufactured product and customer preference to the direct channel are taken into account in the model. The results pointed out that implementing a separate channel can improve the total supply chain's profit compared to the single-channel approach. He et al. [36] explored channel structure and pricing decisions for the manufacturer and government's subsidy policy with competing new and remanufactured products in such dual-channel CLSC. Also, the influence of the two scaling factors abovementioned on the optimal pricing and channel structure are analyzed.

Even though the above literature introduced has studied the optimal pricing decisions for the new and remanufactured products with separate sale channels in the dual-channel CLSC, but most of those works only focus on deriving optimal decision and managerial insights in a static setting (one short game) rather than in a long-term dynamic scenario, which deviates from the actual competition in business and economics in some degree. Choi and Messinger [37] argued that the Stackelberg leadership model generally is intended to describe business situations that involve repeated interactions on real applications. Additionally, Zhang et al. [38] suggested that a game's 
equilibrium decision should be the result of dynamic learning, adjustment and trial \& error.

The dual-channel market describes the structure of the oligarch competition model. The research on the complexity and dynamic behaviors of the oligopoly game model has attracted widespread attention from scholars. In an oligarchic market, the player's optimal decision is chosen based on considering the opponent's reaction, and the players may exert different adjustment mechanisms for their decision variables, viz., naïve, adaptive and bounded rational expectations. There is a vast of work that has enriched the research body of dynamic features of oligarch models [39-40]. The effect of differences between plan products and actual products are analyzed in a Stackelberg model with bounded rationality. Also, the local stability of Nash equilibrium and complex dynamics of the model is studied by numerical simulations in [41]. Askar [42] developed a Cournot duopoly game model with convex and log-concave demand function, and the dynamical characteristics of the model, such as stability, bifurcation and chaos are investigated. A double route to chaotic dynamics via flip bifurcations and Neimark-Sacker bifurcations for a Cournot duopoly game was studied in [43].

Some researchers have established the oligarch models by considering the heterogeneous expectations of the players, and the complex dynamical behaviors of the models are explored [4445]. Wang and Ma [46] studied a Cournot-Bertrand mixed duopoly model with different under the assumption that there is a certain degree of differentiation between the products offed by firms and found that the stability region of the Cournot-Bertrand system is bigger than that of the Cournot or Bertrand system under the same conditions. A nonlinear triopoly game with heterogeneous players was presented in [47], and its bifurcation diagrams, phase portraits and sensitive dependence on initial conditions were shown by numerical simulations. Elsadany et al. [48] investigated a quadropoly game with four heterogeneous firms. Numerical simulations are conducted to illustrate the complex behaviors of the proposed dynamic game as well as the feedback control method is used to control the chaotic behavior.

A few papers have concentrated on studying the application of delay decisions in the long-run decision-making of the bounded rational players. It is confirmed that adopting the delayed decision can make the decision more rational and the system has a higher chance stays in the stability domain. [49-50]. Peng et al. [51] developed a nonlinear dynamic triopoly game model with delayed bounded rationality. The result pointed out that the delay parameters are important factors to eliminate chaos. 
$\mathrm{Li}$ and $\mathrm{Ma}$ [52] considered the delay decision in a dual-channel supply chain. The complex phenomenon of the system and the performance of chain members were studied. Elsadany and Awad [53] proposed a delay monopoly game with bounded rationality. The delay effect on the dynamics of the model was studied. The paper that we have aforementioned above has revealed the effects delay parameters under various frameworks except the CLSC context. Although several pieces of literature have researched the optimal price strategies of the multi-period game in the simple CLSCs [54-55]. Nevertheless, the research on dynamic pricing in the dual-channel CLSC is very scarce.

To the best of our knowledge, our work is the first to study the dynamic pricing game with considering the heterogeneous players adopting the delay decisions in a multi-period Stackelberg game model in the presence of the new and remanufactured products are sold with separate channels. Moreover, based on observations from the extant literature, there exists a theoretical gap that has failed to grasp, that is, how the consumers' behaviors, including the consumer preference degree to the direct channel and the consumer acceptance for the remanufactured product, as well as how the one-step delay decision of the bounded rational manufacturer influences the local stability and complex dynamics of the optimal equilibrium solution, and the performance of chain members under different dynamic motions of the dual-channel CLSC system.

To shed light on the above research questions, we devote ourselves to developing the multiperiod Stackelberg game models in a dual-channel CLSC with considering heterogeneous players and heterogeneous products. Moreover, the effects of the delay factors and the two scaling factors (the consumer acceptance to the direct channel and the consumer discount perception for the remanufactured product) on the system's dynamical evolution are analyzed.

Our paper aims at addressing the following research questions:

(1) How do the two scaling factors influence the long-run optimal pricing strategies and performance of chain members, as well as the local stability and complex dynamic behaviors of the dual-channel CLSC system?

(2) How does the delay decision adopted by the bounded rational manufacturer influence the longrun optimal pricing strategies and performance of chain members, as well as the local stability and complex dynamic behaviors of the dual-channel CLSC system?

(3) How do the price adjustment speed parameters of the heterogeneous players influence the longterm optimal pricing strategies and performance of chain members, as well as the local stability 
and complex dynamic behaviors of the dual-channel CLSC system?

On a broader level, our paper contributes to enriching the research body of the dual-channel CLSC by providing some new managerial insights for managing new and remanufactured products, as well as studies the interactions between heterogeneous players with delay decisions in a noncooperative game model from a dynamic perspective. Also, the chaos control method is extensively applied in the delayed dual-channel CLSC system. It is helpful for the chain members to have a better understanding of the dynamical evolution process of their pricing strategies and performance.

The remainder of the paper is organized as follows. Section 2 introduces model notations and assumptions. The single period Stackelberg game is developed and its optimal solution is derived in Section 3. In section 4, a dynamic Stackelberg game model with non-delay is established, and its stability and dynamical behaviors are investigated. Section 5 proposes a dynamic Stackelberg game model with delay decision, and its complex dynamic behaviors are studied by numerical simulations in section 6. The effects of key parameters on the performance of chain members are analyzed in section 7. Section 8 is chaos control. The conclusions are outlined in section 9 .

\section{Model notations and assumptions}

In the present paper, we study a dual-channel CLSC consisting of one manufacturer (he) and one independent retailer (she). The manufacturer wholesales the new product to the downstream retailer in the traditional channel and sells the remanufactured product in his direct channel to mitigate the cannibalization between new and remanufactured products in the market. The framework of the dual-channel CLSC is displayed as follows:

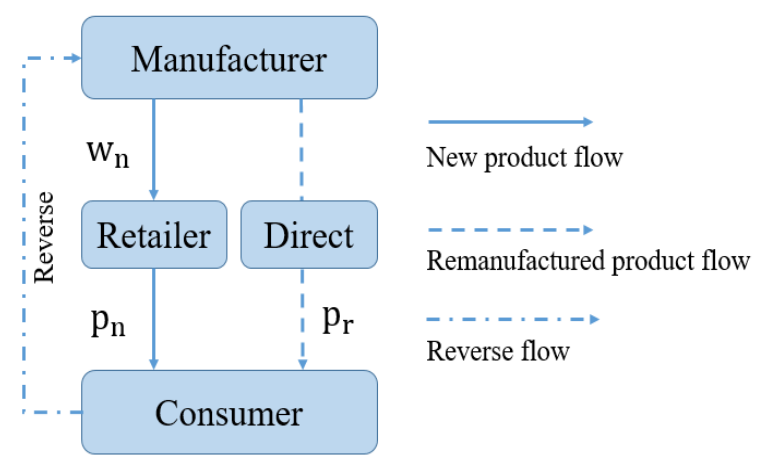

Fig.1. Dual-channel CLSC framework

\subsection{Notation}

We summarize the notations below that will be used throughout the paper. Other notions will 
be introduced when they are needed.

$w_{n}$ the wholesale price for unit new product

$p_{n}$ the retail price for unit new product

$p_{r}$ the retail price for unit remanufactured product

$c_{n}$ the marginal cost for unit new product

$c_{r}$ the marginal cost for unit remanufactured product, $c_{r}<c_{n}$

$\Delta$ the cost saving from unit remanufactured product, $\Delta=c_{n}-c_{r}$

$\delta$ the consumer discount perception for the remanufactured product, $\delta \in(0,1)$

$\theta$ the consumer acceptance to the direct channel, $\theta \in(0,1]$

$\alpha$ the adjustment speed parameter of $w_{n}$

$\beta$ the adjustment speed parameter of $p_{r}$

$v$ the adaptive adjustment parameter of the retailer, $v \in[0,1]$

$w_{i} i=1,2$, the delay factor which denotes the delay weight given to the current period price

$\pi_{j} j=m, r$, the profit of the manufacturer and retailer, respectively

\subsection{Model assumptions}

To establish the models conveniently and simplify, some assumptions are employed in our model.

(1) The manufacturer earns more channel power compared with the retailer in the market, so the manufacturer acts as the leader and the retailer acts as the follower in the Stackelberg game $[26,36]$

(2) The price competition between the channel members is in a discrete-time period $t, t=$ $1,2,3 \ldots$. The business objective of the players is to seek their own profit maximization [54,55].

(3) The manufacturer adopts bounded rationality expectation and the retailer employs adaptive expectation when making their long-term pricing strategies.

(4) Without loss of generality, the total market scale is normalized to 1 . The idiosyncratic valuation of the new product with respect to the willingness-to-pay of consumers is $u$. For analytic simplicity, $u$ is assumed to obey the uniform distribution within the consumer population from 0 to 1 , that is, $u \sim[0,1]$, and the corresponding density function is represented by $f(u)=1[15,36]$

(5) For sake of simplicity, $c_{r}$ is normalized to zero for ease of exposition, then $\Delta=c_{n}[36]$. 


\section{Single period Stackelberg game model}

In this section, we formulate the Stackelberg game in the dual-channel CLSC under a static setting (one short game), where the manufacturer first determines his optimal wholesale price and direct sale price with considering the best reaction of the retailer, then the retailer determines her optimal retail price based on the information announced by the manufacturer.

3.1 The demand function

In the market, the internal competition between the new product and remanufactured products can be regarded as an imperfect substitution. Especially, in the dual-channel CLSC, the consumer can distinguish the remanufactured products from the new products, and also can either purchase the new product from the traditional channel or buy the remanufactured product from the direct channel. In line with the research $[16,33,35]$, the purchase decision of the consumer depends on the consumer surplus received, which equals the valuation minus the price of the product. The purchase decision of the consumers can be classified into the following aspects:

(1) Only the new product is purchased by the consumer when $u-p_{n} \geq 0$ and $u-p_{n}>\theta \delta u-$ $p_{r}$, i.e., $u \geq \frac{p_{n}-p_{r}}{1-\theta \delta}$

(2) Only the remanufactured product is purchased by the consumer when $\theta \delta u-p_{r} \geq 0$ and $u-$ $p_{n}<\theta \delta u-p_{r}$, i.e., $\frac{p_{r}}{\theta \delta} \leq u<\frac{p_{n}-p_{r}}{1-\theta \delta}$. Besides, when $\frac{p_{r}}{\theta \delta}=\frac{p_{n}-p_{r}}{1-\theta \delta}$, that is, $p_{r}=\theta \delta p_{n}$, which leads to no remanufactured product demand by the consumer.

(3) Neither product is purchased by the consumer when $u<\frac{p_{r}}{\theta \delta}$.

According to the above description, the demand for new product can be formulated as:

$$
d_{n}=\int_{\frac{p_{n}-p_{r}}{1-\theta \delta}}^{1} f(u) d u=1-\frac{p_{n}-p_{r}}{1-\theta \delta}
$$

and the demand for remanufactured product can be formulated as:

$$
d_{r}=\int_{\frac{p_{r}}{\theta \delta}}^{\frac{p_{n}-p_{r}}{1-\theta \delta}} f(u) d u=\frac{p_{n}-p_{r}}{1-\theta \delta}-\frac{p_{r}}{\theta \delta}=\frac{\theta \delta p_{n}-p_{r}}{\theta \delta(1-\theta \delta)}
$$

\subsection{Single period Stackelberg game}

Based on above assumptions and conditions, the optimization problem of the manufacturer can be represented by:

$$
\max _{w_{n}, p_{r}} \pi_{m}=p_{r}\left(\frac{\theta \delta p_{n}-p_{r}}{\theta \delta(1-\theta \delta)}\right)+\left(w_{n}-\Delta\right)\left(1-\frac{p_{n}-p_{r}}{1-\theta \delta}\right)
$$

and the optimization problem of the retailer can be represented by: 


$$
\max _{p_{n}} \pi_{r}=\left(p_{n}-w_{n}\right)\left(1-\frac{p_{n}-p_{r}}{1-\theta \delta}\right)
$$

The subgame Nash equilibrium solution can be achieved by employing the backward induction approach.

Proposition 1. The single period Stackelberg game exists a unique optimal solution, which given by:

$$
\begin{gathered}
w_{n}^{*}=\frac{1+\Delta}{2} \\
p_{r}^{*}=\frac{\delta \theta}{2} \\
p_{n}^{*}=\frac{3+\Delta-\delta \theta}{4}
\end{gathered}
$$

Proof 1: Taking the first-order condition of $\pi_{r}$ with respect to $p_{r}$, we have:

$$
\frac{\partial \pi_{r}}{\partial p_{n}}=\frac{1-2 p_{n}+p_{r}+w_{n}-\delta \theta}{1-p_{r}}
$$

Thus, the best response function of the retailer is

$$
p_{n}=\frac{1+p_{r}+w_{n}-\delta \theta}{2}
$$

Plugging the above function into $\pi_{m}$ and taking the first-order partial derivatives of $\pi_{m}$ with respect to $w_{n}$ and $p_{r}$ respectively, then we have

$$
\begin{gathered}
\frac{\partial \pi_{m}}{\partial w_{n}}=\frac{1+2 p_{r}-2 w_{n}+\Delta-\delta \theta}{2(1-\delta \theta)} \\
\frac{\partial \pi_{m}}{\partial p_{r}}=\frac{1}{2}\left(1-\frac{4 p_{r}}{\delta \theta}-\frac{2 p_{r}-2 w_{n}+\Delta}{1-\delta \theta}\right)
\end{gathered}
$$

The corresponding Hessian matrix of $\pi_{m}$ with respect to $w_{n}$ and $p_{r}$ can be computed as follows:

$$
\begin{gathered}
H_{m}=\left[\begin{array}{cc}
\frac{\partial^{2} \pi_{m}}{\partial w_{n}^{2}} & \frac{\partial^{2} \pi_{m}}{\partial w_{n} \partial p_{r}} \\
\frac{\partial^{2} \pi_{m}}{\partial p_{r} \partial w_{n}} & \frac{\partial^{2} \pi_{m}}{\partial p_{r}^{2}}
\end{array}\right] \\
=\left[\begin{array}{cc}
-\frac{1}{1-\delta \theta} & \frac{1}{1-\delta \theta} \\
\frac{1}{1-\delta \theta} & -\frac{2-\delta \theta}{\delta \theta(1-\delta \theta)}
\end{array}\right]
\end{gathered}
$$

By observing the above Hessian matrix, the first-order principal minors $H_{m}^{1}=-\frac{1}{1-\delta \theta}<0$ and the second-order principal minors $H_{m}^{2}=\frac{2}{\delta \theta-\delta^{2} \theta^{2}}>0$. Hence, the $H_{m}$ is negative definite matrix and $\pi_{m}$ is jointly concave in $w_{n}$ and $p_{r}$. Therefore, the optimal channel prices of the manufacturer can be obtained by setting $\frac{\partial \pi_{m}}{\partial w_{n}}=\frac{\partial \pi_{m}}{\partial p_{r}}=0$, then we have:

$$
w_{n}=\frac{1+\Delta}{2}, p_{r}=\frac{\delta \theta}{2}
$$

Substituting the Equation (11) into (7), then the optimal retail price of the new product can be calculated, i.e., $p_{n}=\frac{3+\Delta-\delta \theta}{4}$. Thus far, the optimal solution of the Stackelberg game is realized. 
Consequently, the optimal profit functions of the manufacturer and retailer can be obtained as follows:

$$
\begin{gathered}
\pi_{r}=\frac{(1-\Delta-\delta \theta)^{2}}{16(1-\delta \theta)} \\
\pi_{m}=\frac{(1-\Delta)^{2}+2 \delta \Delta \theta-\delta^{2} \theta^{2}}{8(1-\delta \theta)}
\end{gathered}
$$

\section{Dynamic Stackelberg game model without delay}

In this section, we focus on extending the single-period Stackelberg game to a multi-period Stackelberg game by considering the heterogeneous expectations of the channel members. In reality, the decision-makers update their product prices based on the information grasped in the market. However, having limited information collecting and processing ability and so on, the players can impossibly capture all the information that they need for decision making. Alternatively, they often make price decisions based on partial information. Herein, we assume that the manufacturer determines his next-period decision with bounded rationality rule, i.e., when the marginal profit of the current period is positive(negative), the price charged by him will be raised (decreased) in the next period. Moreover, the retailer is supposed to be an adaptive player who makes her next period price based on the best reply function and the current period price. Hence, the discrete dynamic adjustment mechanism of the dual-channel CLSC system is given by:

$$
\left\{\begin{array}{l}
w_{n}(t+1)=w_{n}(t)+\alpha w_{n}(t) \frac{\partial \pi_{m}}{\partial w_{n}} \\
p_{r}(t+1)=p_{r}(t)+\beta p_{r}(t) \frac{\partial \pi_{m}}{\partial p_{r}} \\
p_{n}(t+1)=v\left(\frac{1+p_{r}(t+1)+w_{n}(t+1)-\delta \theta}{2}\right)+(1-v) p_{n}(t)
\end{array}\right.
$$

where $\frac{\partial \pi_{m}}{\partial w_{n}}$ and $\frac{\partial \pi_{m}}{\partial p_{r}}$ are the marginal profits, they can be obtained by (8) and (9). Consequently, the above discrete dynamic system can be rewritten as:

$$
\left\{\begin{array}{c}
w_{n}(t+1)=w_{n}(t)+\alpha w_{n}(t)\left(\frac{1+2 p_{r}(t)-2 w_{n}(t)+\Delta-\delta \theta}{2(1-\delta \theta}\right) \\
p_{r}(t+1)=p_{r}(t)+\beta \frac{p_{r}(t)}{2}\left(1-\frac{4 p_{r}(t)}{\delta \theta}-\frac{2 p_{r}(t)-2 w_{n}(t)+\Delta}{1-\delta \theta}\right) \\
p_{n}(t+1)=v\left(\frac{1+p_{r}(t+1)+w_{n}(t+1)-\delta \theta}{2}\right)+(1-v) p_{n}(t)
\end{array}\right.
$$

Specifically, if $v=0$, the retail price charged by retailer would never change, and if $v=1$, it would be equal to the best response with naïve expectation. 


\subsection{Equilibrium points and local stability}

In order to study the qualitative behavior of the equilibrium solutions of the dynamic system (15), we define the equilibrium points of the dynamic Stackelberg game as nonnegative fixed points. In what follows, imposing $w_{n}(t+1)=w_{n}(t), p_{r}(t+1)=p_{r}(t), p_{n}(t+1)=p_{n}(t)$, and we can get four equilibrium points which given by:

$$
\begin{aligned}
& E_{1}=\left(0,0, \frac{1-\delta \theta}{2}\right), \\
& E_{2}=\left(0, \frac{\delta \theta(1-\delta \theta-\Delta)}{2(2-\delta \theta)}, \frac{4-\delta \theta(4-\Delta)}{4(2-\delta \theta)}\right), \\
& E_{3}=\left(\frac{1+\Delta-\delta \theta}{2}, 0, \frac{3(1-\delta \theta)+\Delta}{4}\right), \\
& E_{4}=\left(w_{n}^{*}, p_{r}^{*}, p_{n}^{*}\right) .
\end{aligned}
$$

To guarantee all the equilibrium points are nonnegative coordinates, the inequality $1-\delta \theta-$ $\Delta>0$ should be hold. Obviously, the $E_{1}, E_{2}, E_{3}$ are all boundary equilibrium points, and only $E_{4}$ is Nash equilibrium point.

To investigate the local stability of the equilibrium points, we estimate the Jacobian matrix of the discrete dynamic system (15) which is given by:

$$
J=\left[\begin{array}{ccc}
1+\frac{\alpha\left(1+2 p_{r}-4 w_{n}+\Delta-\delta \theta\right)}{2-2 \delta \theta} & \frac{-\alpha w_{n}}{1-\delta \theta} & 0 \\
\frac{\beta p_{r}}{1-\delta \theta} & 1+\frac{\beta}{2}\left(1-\frac{8 p_{r}}{\delta \theta}-\frac{4 p_{r}-2 w_{n}+\Delta}{1-\delta \theta}\right) & 0 \\
\frac{v}{2} & \frac{v}{2} & 1-v
\end{array}\right]
$$

The stability condition to the equilibrium points require all its the characteristic roots evaluated at the corresponding Jacobian matrix should be in the unit disk, i.e., $\left|\lambda_{i}\right|<1, i=1,2,3$.

Proposition 2. The trivial equilibrium points $E_{1}, E_{2}, E_{3}$ of the system (15) are unstable points

Proof 2. The Jacobian matrix at the equilibrium point $E_{1}$ is

$$
J\left(E_{1}\right)=\left[\begin{array}{ccc}
1+\frac{\alpha(1+\Delta-\delta \theta)}{2-2 \delta \theta} & 0 & 0 \\
0 & 1+\frac{\beta(1-\delta \theta-\Delta)}{2(1-\delta \theta)} & 0 \\
\frac{v}{2} & \frac{v}{2} & 1-v
\end{array}\right]
$$

It is clear that the above matrix is a triangular matrix, and the eigenvalues of it can be easily computed, that is, $\lambda_{1}=1+\frac{\alpha(1+\Delta-\delta \theta)}{2(1-\delta \theta)}, \lambda_{2}=1+\frac{\beta(1-\delta \theta-\Delta)}{2(1-\delta \theta)}$ and $\lambda_{3}=1-v$. Since all the model parameters are nonnegative constants, then $\left|\lambda_{1,2}\right|>1$ and $\left|\lambda_{3}\right|<1$ are verified. Thus, the $E_{1}$ is a saddle point (unstable node).

As for $E_{2}$, the corresponding Jacobian matrix can be calculated as: 


$$
J\left(E_{2}\right)=\left[\begin{array}{ccc}
1+\frac{\alpha(1+\Delta)}{2-\delta \theta} & 0 & 0 \\
\frac{\beta \delta(1-\delta \theta-\Delta)}{2(2-\delta \theta)(1-\delta \theta)} & 1-\frac{\beta(1-\delta \theta-\Delta)}{2(1-\delta \theta)} & 0 \\
\frac{v}{2} & \frac{v}{2} & 1-v
\end{array}\right]
$$

which has eigenvalues $\lambda_{1}=1+\frac{\alpha(1+\Delta)}{2-\delta \theta}, \lambda_{2}=1-\frac{\beta(1-\delta \theta-\Delta)}{2(1-\delta \theta)}$ and $\lambda_{3}=1-v$. It is easily can judge that $\left|\lambda_{1}\right|>1$ and $\left|\lambda_{2,3}\right|<1$, so $E_{2}$ is an unstable node. In the similarly way, the eigenvalues of Jacobian matrix evaluated at $E_{3}$ can be computed as $\lambda_{1}=1+\beta, \lambda_{2}=1-\frac{\alpha(1+\Delta-\delta \theta)}{1-\delta \theta}$ and $\lambda_{3}=1-v$. Therefore, the $E_{3}$ is also an unstable node for $\left|\lambda_{1}\right|>1$.

As the boundary equilibria, $E_{1}, E_{2}, E_{3}$ are stand for the decision makers' short-term behavior or one of the plays withdrawing from the market. Next, in order to investigate the asymptotic stability of the Nash equilibrium, the Jacobian matrix calculated at $E_{4}$ is given by:

$$
J\left(E_{4}\right)=\left[\begin{array}{ccc}
1+\frac{\alpha\left(1+2 p_{r}^{*}-4 w_{n}^{*}+\Delta-\delta \theta\right)}{2-2 \delta} & \frac{-\alpha w_{n}^{*}}{1-\delta \theta} & 0 \\
\frac{\beta p_{r}^{*}}{1-\delta \theta} & 1+\frac{\beta}{2}\left(1-\frac{8 p_{r}^{*}}{\delta \theta}-\frac{4 p_{r}^{*}-2 w_{n}^{*}+\Delta}{1-\delta \theta}\right) & 0 \\
\frac{v}{2} & \frac{v}{2} & 1-v
\end{array}\right]
$$

whose characteristic polynomial takes the form:

$$
f(\lambda)=\lambda^{3}+A \lambda^{2}+B \lambda+C
$$

where $A=(1-v)\left(-\left(1+\frac{1}{2} \beta\left(1-\frac{8 p_{r}^{*}}{\delta \theta}-\frac{\Delta+4 p_{r}^{*}-2 w_{n}^{*}}{1-\delta \theta}\right)\right)\left(1+\frac{\alpha\left(1+\Delta-\delta \theta+2 p_{r}^{*}-4 w_{n}^{*}\right)}{2-2 \delta \theta}\right)-\frac{\alpha \beta p_{r}^{*} w_{n}^{*}}{(-1+\delta \theta)^{2}}\right)$,

$B=(1-v)\left(1+\frac{1}{2} \beta\left(1-\frac{8 p_{r}^{*}}{\delta \theta}+\frac{\Delta+4 p_{r}^{*}-2 w_{n}^{*}}{-1+\delta \theta}\right)+\left(1+\frac{\alpha\left(1+\Delta-\delta \theta+2 p_{r}^{*}-4 w_{n}^{*}\right)}{2-2 \delta \theta}\right)\right)+\left(1+\frac{1}{2} \beta\left(1-\frac{8 p_{r}^{*}}{\delta \theta}-\right.\right.$

$\left.\left.\frac{\Delta+4 p_{r}^{*}-2 w_{n}^{*}}{1-\delta \theta}\right)\right)\left(1+\frac{\alpha\left(1+\Delta-\delta \theta+2 p_{r}^{*}-4 w_{n}^{*}\right)}{2-2 \delta \theta}\right)+\frac{\alpha \beta p_{r}^{*} w_{n}^{*}}{(-1+\delta \theta)^{2}}$,

$C=-3+v-\frac{1}{2} \beta\left(1-\frac{8 p_{r}^{*}}{\delta \theta}-\frac{\Delta+4 p_{r}^{*}-2 w_{n}^{*}}{1-\delta \theta}\right)-\frac{\alpha\left(1+\Delta-\delta \theta+2 p_{r}^{*}-4 w_{n}^{*}\right)}{2-2 \delta \theta}$.

According to the Jury conditions [56], the sufficient and necessary conditions that the Nash equilibrium point is locally stable are

$$
\left\{\begin{array}{l}
f(1)=A+B+C+1>0 \\
-f(-1)=-A+B-C+1>0 \\
C^{2}-1<0 \\
\left(1-C^{2}\right)^{2}-(B-A C)^{2}>0
\end{array}\right.
$$

The condition (22) gives a stable region in the place of the adjustment parameters $\alpha, \beta$ and $v$. The economic meaning of the stability region can be explained that whatever initial channel prices are chosen by the decision-makers in the local stable region, the market will eventually reach the Nash equilibrium point after finite games.

When the above inequalities are failed to satisfy simultaneously can prompt different types of 
bifurcation to take place. If there exists a real eigenvalue smaller than -1 , then a flip bifurcation will appear, and if the modulus of the complex eigenvalue is bigger than +1 , which will lead to a Neimark-Sacker bifurcation occurs.

4.2 The effects of key parameters on the stable region of system (15)

To study the influence of some key parameters on the stability domain of the system (15), it is convenient to take the following data set: $\Delta=0.1, w_{n}(1)=0.5, p_{r}(1)=0.3, p_{n}(1)=0.6$.

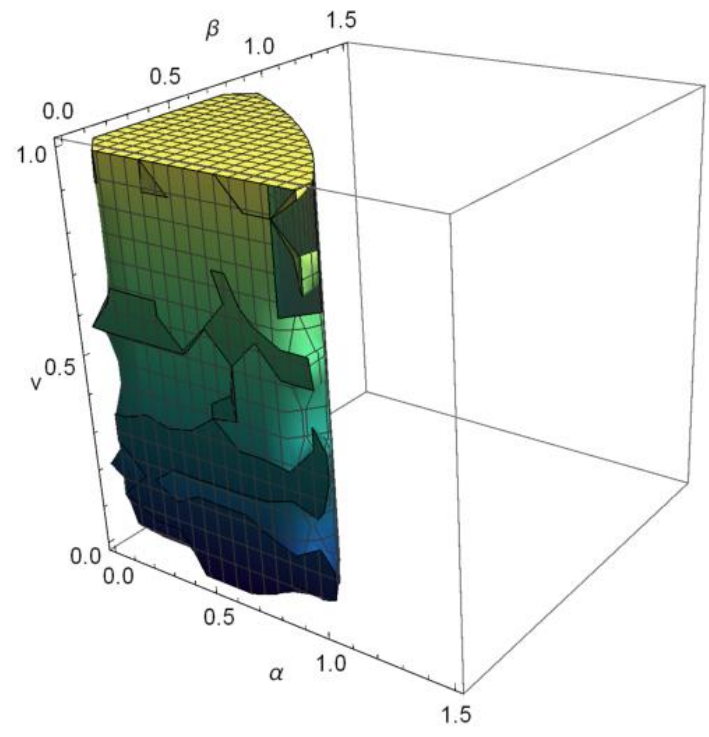

Fig.2. The 3D stable region in terms of $(\alpha, \beta, v)$ when $\delta=0.8, \theta=0.9$

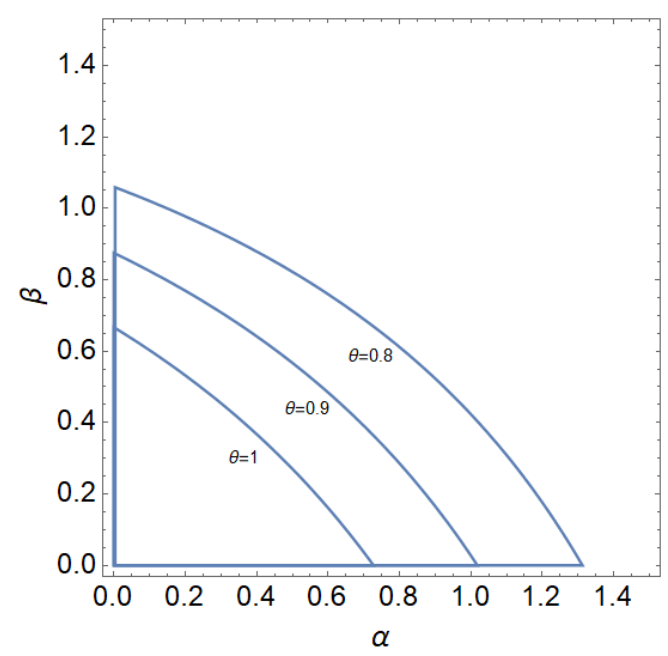

(a) $\delta=0.8, v=0.5$

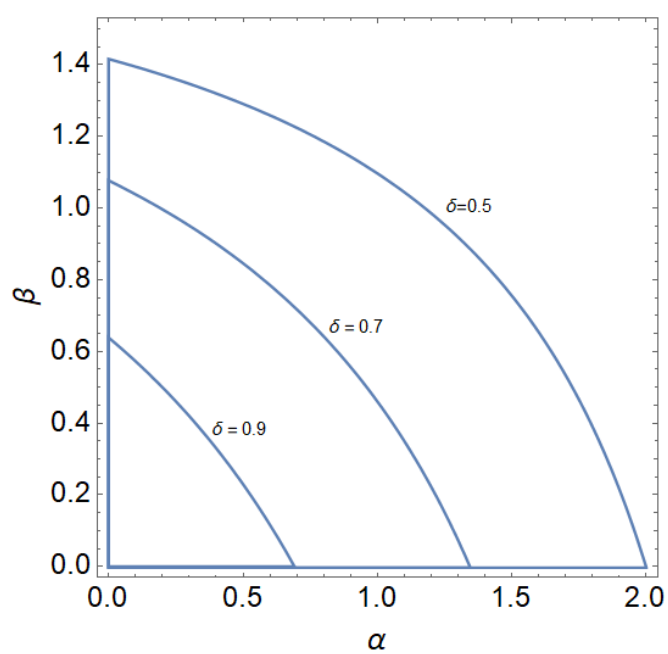

(b) $\theta=0.9, v=0.5$

Fig. 3. The influence of $\theta$ and $\delta$ on the stable region with the plane of $(\alpha, \beta)$

Fig. 2 shows a three-dimensional stable region of the system (15), which is locates within the value of $(\alpha, \beta, v)$. The Nash equilibrium price will be reached after several games when all the 
values of the adjustment speed parameters are located in the stable region. However, once the value of $\alpha$ or $\beta$ gets rid of the stable region, the system tends to lose its stability, as well as more complex dynamic behaviors of price evolution will occur such as bifurcation and chaos. Hence, the adjustment speed parameters of manufacturer can exert a significant impact on the system's stability. Besides, by varying $v$ from 0 to 1 when $\alpha$ and $\beta$ are fixed at their small values within the stable region, one can observe that the size of the system's stable region almost unchanged, which indicates the adaptive adjustment parameter of retailer has an extremely slight effect on the system's stability.

The change of stable regions with different values of $\theta$ is plotted in Fig.3(a). We assign 0.8, $0.9,1$ to $\theta$ respectively, and the size of the stable region gradually becomes smaller, which implies that the higher level of the consumer preference to the direct channel, the narrower the system's stable region. Hence, we can draw the conclusion that a higher acceptance degree to the direct channel of the consumer has a negative impact on the system stability due to it has enhanced the competition intensity between the conventional retail channel and direct channel.

Fig.3(b) is displayed to detect the impact of $\delta$ on the system's stability for the parameter values $\theta=0.8, v=0.5$. As can be seen that as $\delta$ tends to be larger, the size of the stable region is shrinkage to some extent, which indicates the higher level of the consumer discount perception for remanufactured products, the system is more likely to break out of the stable region. Therefore, one can deduce that a greater valuation for the remanufactured product charged by the consumer in direct channel has a detrimental effect on the system stability. From the above numerical analysis, we can find that either the higher level of the consumer preference degree to the direct channel or consumer valuation for the remanufactured product can lead to the more intense cannibalization between the new and remanufactured products in the dual-channel CLSC. Naturally, the system has a higher chance gets into the unstable region. 


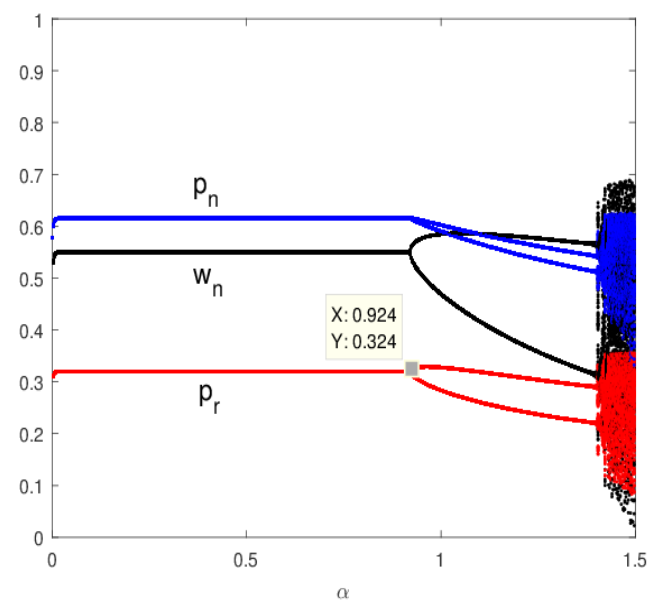

(a)

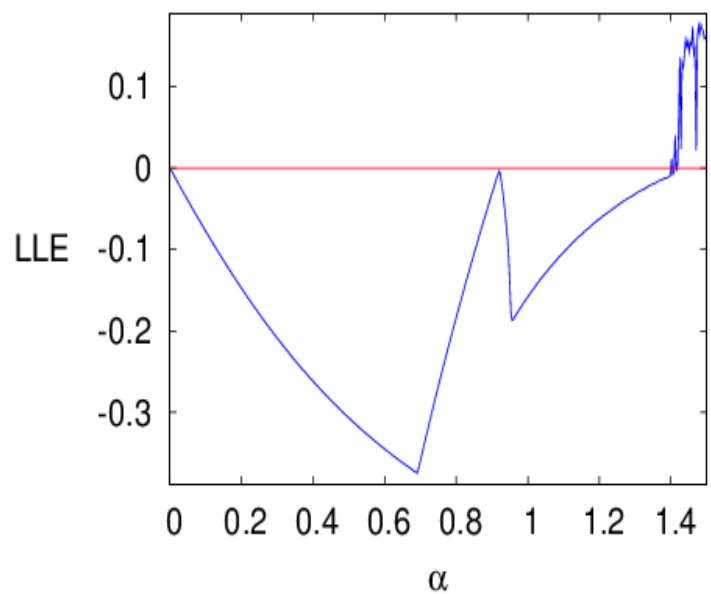

(b)

Fig.4. Bifurcation diagram with respect to $\alpha$ and the corresponding LLE diagram when $\beta=v=$ 0.5 .

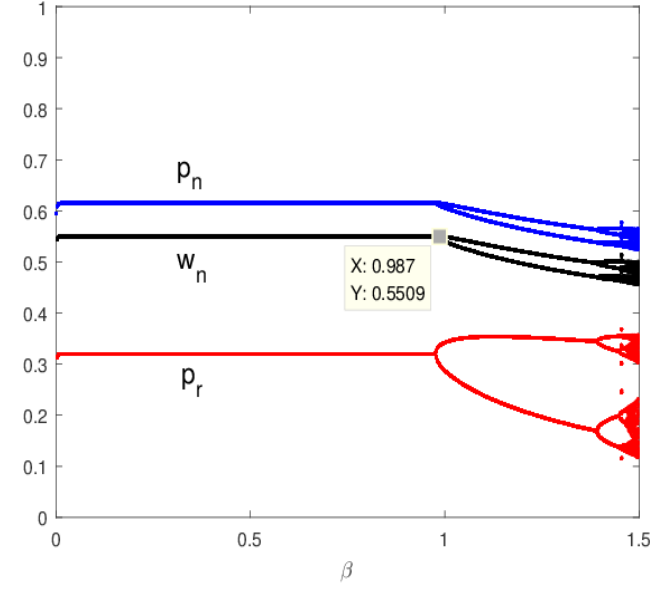

(a)

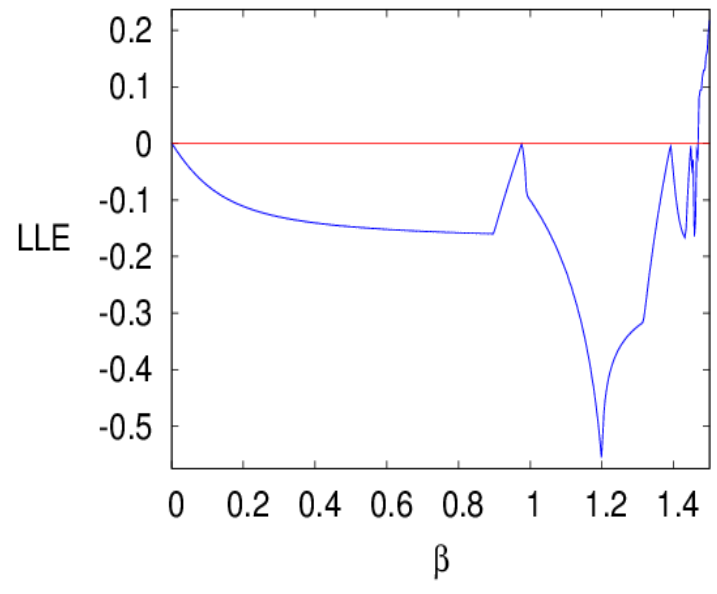

(b)

Fig.5. Bifurcation diagram with respect to $\beta$ and the corresponding LLE diagram when $\alpha=$ $v=0.5$.
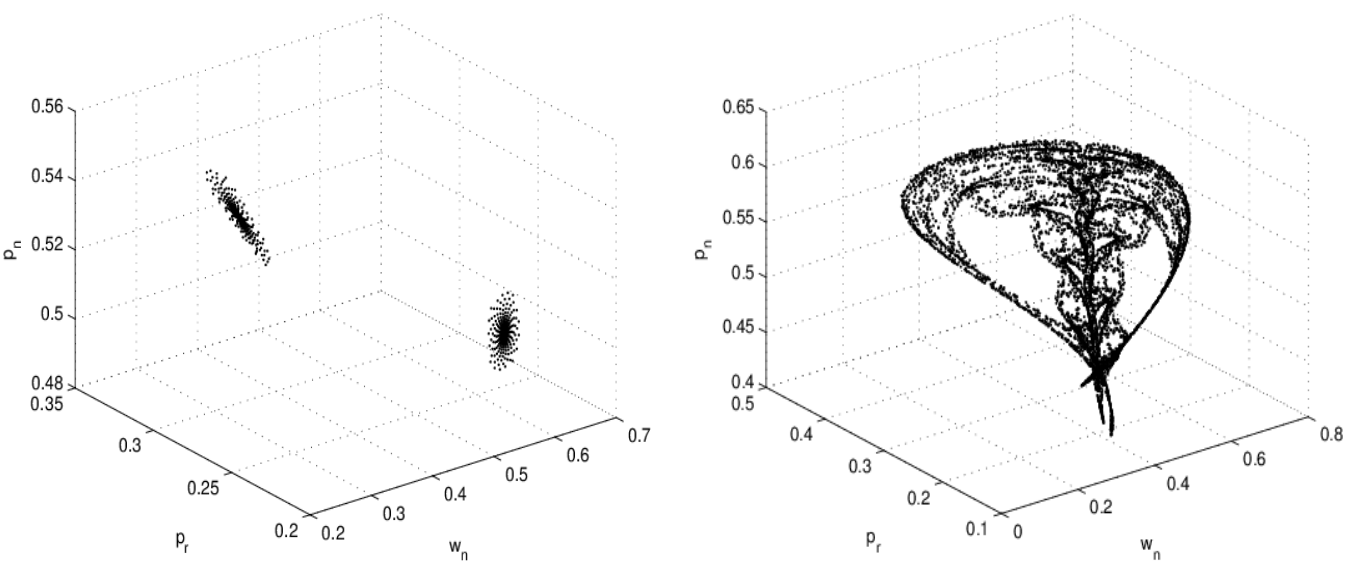
Fig.6. Strange attractors with (a) $\alpha=1.429, \beta=v=0.5$; (b) $\alpha=1.435, \beta=v=0.5$.

4.3 Bifurcations and chaotic behaviors

This subsection is devoted to providing some numerical simulations to explore the effects of adjustment speed parameters on the long-run dynamic behaviors of the system (15). In Fig.4(a), the bifurcation diagram of the system (15) is shown when $\alpha$ is taken as a dynamical parameter and the other parameters are fixed as $\beta=v=0.5$ and $\delta=\theta=0.8$. One can see that the system converges to the Nash equilibrium point when $\alpha<0.924$, and then a period doubling bifurcation takes place when $\alpha$ is about to 0.924 . After that, the system falls into chaotic state eventually through the Neimark-Sacker bifurcation as $\alpha$ increases. The corresponding largest Lyapunov exponents associated with Fig.4(a) are presented in Fig.4(b), whereas the negative LLEs indicate the system is stable, and the positive LLEs can verify the existence of chaotic behaviors.

Fig.5(a) describes the price orbits with varying $\beta$ from 0 to 1.5 . One can see that the first bifurcation point appears when $\beta$ approaching 0.987 . Finally, the system comes into chaos by undergoing a series of period doubling bifurcations and the corresponding LLE diagram is drawn in Fig.5(b).

Fig.6 reports some strange attractors with different values of $\alpha$ when $\beta$ and $v$ are fixed at 0.5. The two chaotic unconnected areas are built up when $\alpha=1.429$ in Fig.6(a) which evolves in a more complicated chaotic area with a great value $\alpha=1.435$ as shown in Fig.6(b).

From the above numerical experiments, we can draw the conclusion that the excessive fast price adjustment speed can lead to the market structure behaving chaotically. Especially, in the real market, the decision-makers are willing to capture more profits by accelerating the adjustment speed of product prices. Once the players adjust their channel prices too fast which will push the system gets into chaotic and the market becomes disorder and unpredictable. Under such a case, it is difficult for them to make a good long-term price strategy to achieve profit maximization. Therefore, the players in the CLSC should be prudent to choose a proper price adjustment speed to control the system in a stable state. It is noticed that the adjustment speed of price will not change the value of Nash equilibria. 


\section{Dynamic Stackelberg game model with delay}

In the repeated game, the bounded rational player trends to charge his next period price $p^{e}(t+1)$ utilizing a prediction feedback by comprehensively considering the current price $p(t)$ and the previous prices $p(t-1), p(t-2), \ldots, p(t-T)$ with different weights. Doing this will make the decision more rational from the perspective of economic. Therefore, we consider combining bounded rationality with one-period delay decision for the manufacturer to update his long-run price strategy in the dual-channel CLSC, i.e., the expected wholesale price and direct price in the next period are formulated as $w_{n}^{e}(t+1)=w_{1} w_{n}(t)+\left(1-w_{1}\right) w_{n}(t-1)$ and $p_{r}^{e}(t+$ 1) $=w_{2} p_{r}(t)+\left(1-w_{2}\right) p_{r}(t-1)$ respectively.

Accordingly, the discrete dynamic system with delay decisions can be given by:

$$
\left\{\begin{array}{l}
w_{n}(t+1)=w_{n}(t)+\alpha w_{n}(t)\left(\frac{1+2\left(w_{2} p_{r}(t)+\left(1-w_{2}\right) p_{r}(t-1)\right)-2\left(w_{1} w_{n}(t)+\left(1-w_{1}\right) w_{n}(t-1)\right)+\Delta-\delta \theta}{2(1-\delta \theta)}\right) \\
p_{r}(t+1)=p_{r}(t)+\beta \frac{p_{r}(t)}{2}\left(1-\frac{4\left(w_{2} p_{r}(t)+\left(1-w_{2}\right) p_{r}(t-1)\right)}{\delta \theta}-\frac{2\left(w_{2} p_{r}(t)+\left(1-w_{2}\right) p_{r}(t-1)\right)-2\left(w_{1} w_{n}(t)+\left(1-w_{1}\right) w_{n}(t-1)\right)+\Delta}{1-\delta \theta}\right) \\
p_{n}(t+1)=v\left(\frac{1+\left(w_{1} w_{n}(t)+\left(1-w_{1}\right) w_{n}(t-1)\right)+\left(w_{2} p_{r}(t)+\left(1-w_{2}\right) p_{r}(t-1)\right)-\delta \theta}{2}\right)+(1-v) p_{n}(t)
\end{array}\right.
$$

where the delay factor $0 \leq w_{i} \leq 1, i=1,2$ represents the delay weights given to $t$ period by manufacturer. It is notice that when $w_{i}=1$, the delayed system (23) turns into the system (15).

To study the dynamic system (23) conveniently, it can be rewritten as a 5-dimensional system by letting $x(t+1)=w_{n}(t), y(t+1)=p_{r}(t)$ as follows:

$$
\left\{\begin{array}{c}
w_{n}(t+1)=w_{n}(t)+\alpha w_{n}(t)\left(\frac{1+2\left(w_{2} p_{r}(t)+\left(1-w_{2}\right) y(t)\right)-2\left(w_{1} w_{n}(t)+\left(1-w_{1}\right) x(t)\right)+\Delta-\delta \theta}{2(1-\delta \theta)}\right) \\
p_{r}(t+1)=p_{r}(t)+\beta \frac{p_{r}(t)}{2}\left(1-\frac{4\left(w_{2} p_{r}(t)+\left(1-w_{2}\right) y(t)\right)}{\delta \theta}-\frac{2\left(w_{2} p_{r}(t)+\left(1-w_{2}\right) y(t)\right)-2\left(w_{1} w_{n}(t)+\left(1-w_{1}\right) x(t)\right)+\Delta}{1-\delta \theta}\right) \\
p_{n}(t+1)=v\left(\frac{1+\left(w_{1} w_{n}(t)+\left(1-w_{1}\right) x(t)\right)+\left(w_{2} p_{r}(t)+\left(1-w_{2}\right) y(t)\right)-\delta \theta}{2}\right)+(1-v) p_{n}(t) \\
x(t+1)=w_{n}(t) \\
y(t+1)=p_{r}(t)
\end{array}\right.
$$

By making $w_{n}(t+1)=w_{n}(t), p_{r}(t+1)=p_{r}(t), p_{n}(t+1)=p_{n}(t), x(t+1)=x(t)$, $y(t+1)=y(t)$, then the four equilibrium points can be obtained as below:

$E_{1}=\left(0,0, \frac{1-\delta \theta}{2}, 0,0\right)$

$E_{2}=\left(0, \frac{\delta \theta(1-\delta \theta-\Delta)}{2(2-\delta \theta)}, \frac{4-\delta \theta(4-\Delta)}{4(2-\delta \theta)}, 0, \frac{\delta \theta(1-\delta \theta-\Delta)}{2(2-\delta \theta)}\right)$,

$E_{3}=\left(\frac{1+\Delta-\delta \theta}{2}, 0, \frac{3(1-\delta \theta)+\Delta}{4}, \frac{1+\Delta-\delta \theta}{2}, 0\right)$,

$E_{4}=\left(w_{n}^{*}, p_{r}^{*}, p_{n}^{*}, w_{n}^{*}, p_{r}^{*}\right)$.

Due to the delay parameter is irrelevant to the value of the equilibrium solution, so it is clear that the $E_{1}, E_{2}, E_{3}$ are unstable nodes, and only the $E_{4}$ is a unique subgame perfect Nash equilibrium. In the following section, the dynamics properties of the system (24) under various 
conditions will be discussed by numerical simulation.

\section{Numerical simulations}

This section we are concentrate on providing some numerical experiments to show the key parameters on the complex dynamics of the delayed system (24).

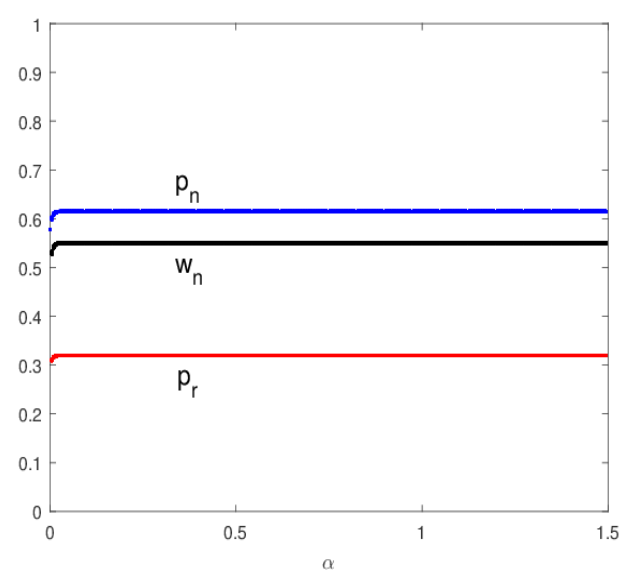

(a) $w_{1}=w_{2}=0.8$

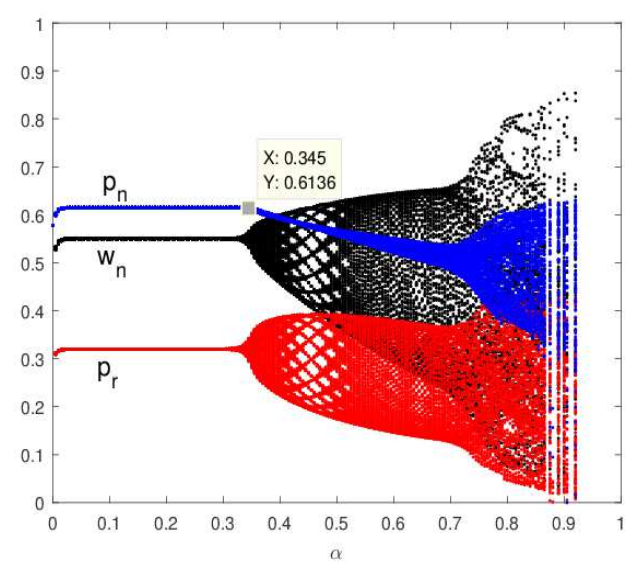

(c) $w_{1}=w_{2}=0.2$

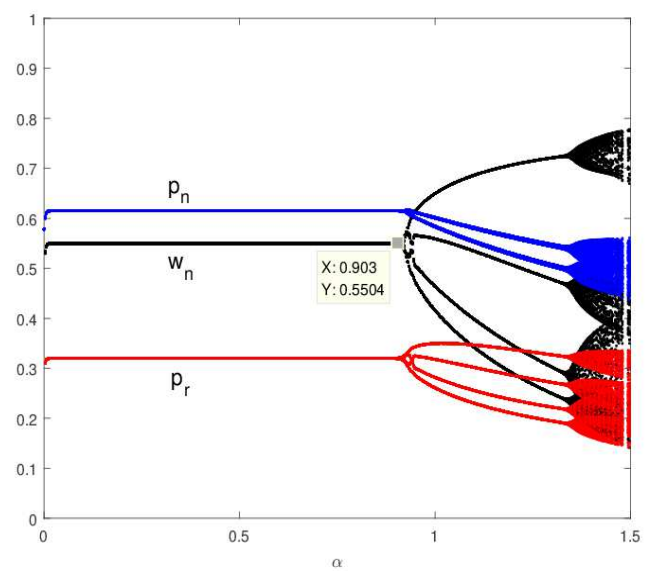

(b) $w_{1}=w_{2}=0.5$

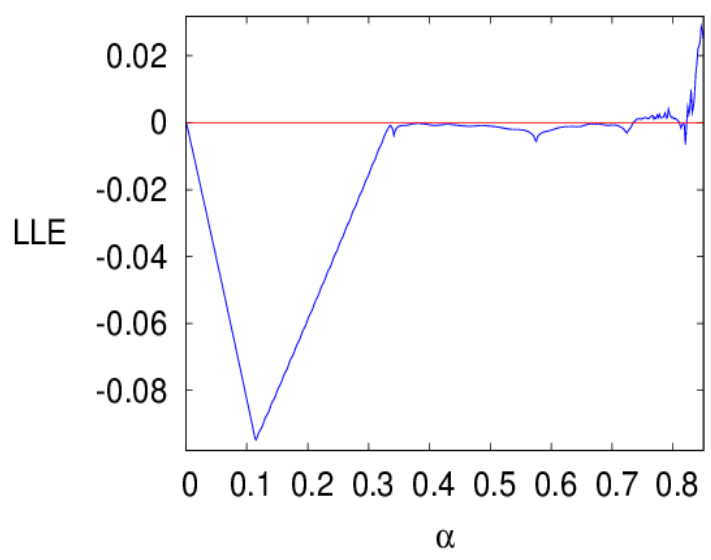

(d) $w_{1}=w_{2}=0.2$

Fig.7. Bifurcation diagrams with respect to $\alpha$ under different delay weights when $\beta=v=0.5$.

6.1 The influence of key parameters on the system's dynamical evolution

Fig.7(a)-(c) displays the price trajectory diagrams with the change of $\alpha$ under three different delay parameter values, that is, $w_{i}=0.8,0.5,0.2$, respectively. One can observe that the system is always fixed at the Nash equilibrium point when $w_{i}=0.8$. When taking delay weights values $w_{i}=0.5$, the channel prices converge at Nash equilibrium when $\alpha<0.903$, then a stable period four-cycle appears with a greater value of $\alpha$, and price trajectories become chaotic eventually via a Neimark-Sacker bifurcation along with $\alpha$ increasing. Besides, the bifurcation diagram with $w_{i}=$ 
0.2 is performed as shown in Fig.7(c). The Nash equilibrium is locally stable for $\alpha<0.345$, and loss its stability through Neimark-Sacker bifurcation at $\alpha=0.345$, when $\alpha$ exceeds 0.345 , which give birth to an attracting invariant circle and chaotic behavior finally. Also, the LLEs which are associated with Fig.7(c) is disposed in Fig.7(d). As can be seen from Fig.7(d), we notice that the LLEs are fluctuating around zero when a Neimark-Sacker bifurcation appears. Meanwhile, the dynamic motion of the market changes into a quasiperiodic state with limit cycles. Under this condition, it is more difficult to adopt a good long-term pricing strategy for the decision-makers but a quasiperiodic time series still makes such things possible as useful approximation. Furthermore, when the system falls into a chaotic state, the market will become irregular and unpredictable, it is extremely impossible for the players to deal with such circumstances.

In addition, by comparing the delayed cases in Fig.7 and without delay case in Fig.4(a), we can find that period-doubling bifurcation and chaos periods of Nash equilibrium are eliminated by choosing $w_{i}=0.8$, while the Nash equilibrium is more easily loses its stability due to the perioddoubling or Neimark-Sacker bifurcation occurs earlier when setting $w_{i}$ equal to 0.5 and 0.2 respectively. Hence, the conclusion can be drawn that an appropriate weight given to the delay factors has a strong stabilization effect for the Nash equilibrium point by expending its stable region, while an unsuitable delay weight can exert a great destabilization effect on the system's dynamical evolution. In other words, the delay decision utilized by the manufacturer may have a positive or negative impact on the system stability.

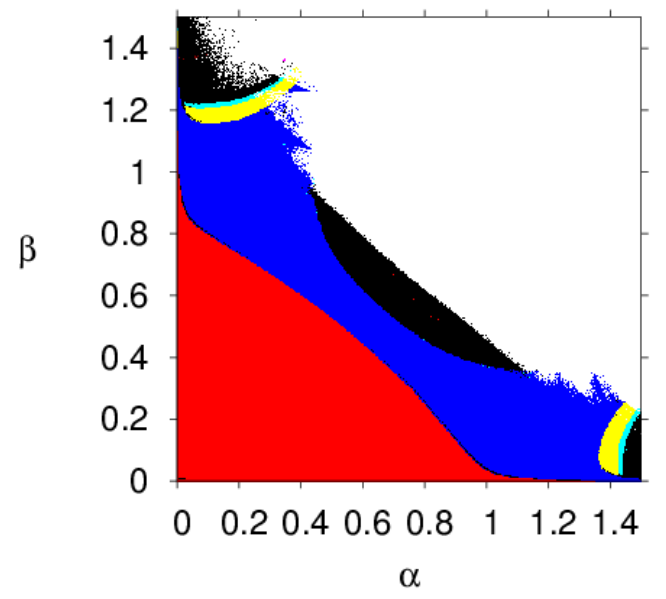

(a) $w_{1}=w_{2}=1$

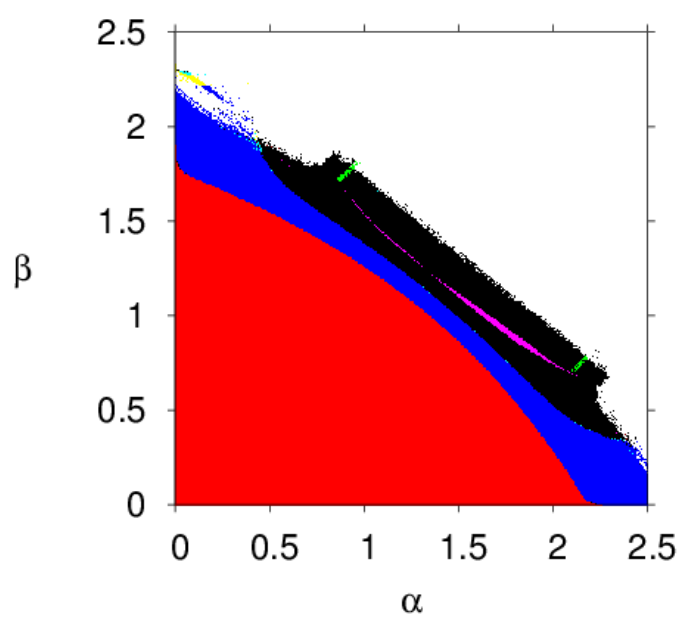

(b) $w_{1}=w_{2}=0.8$ 


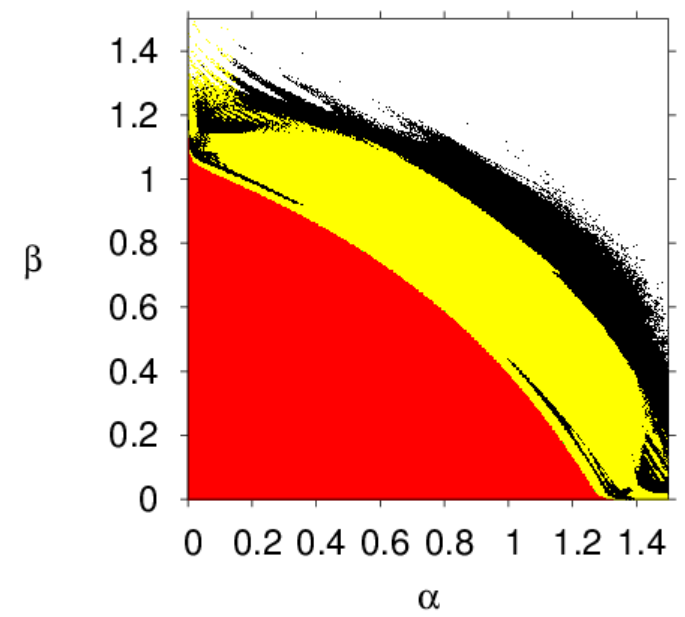

(c) $w_{1}=w_{2}=0.5$

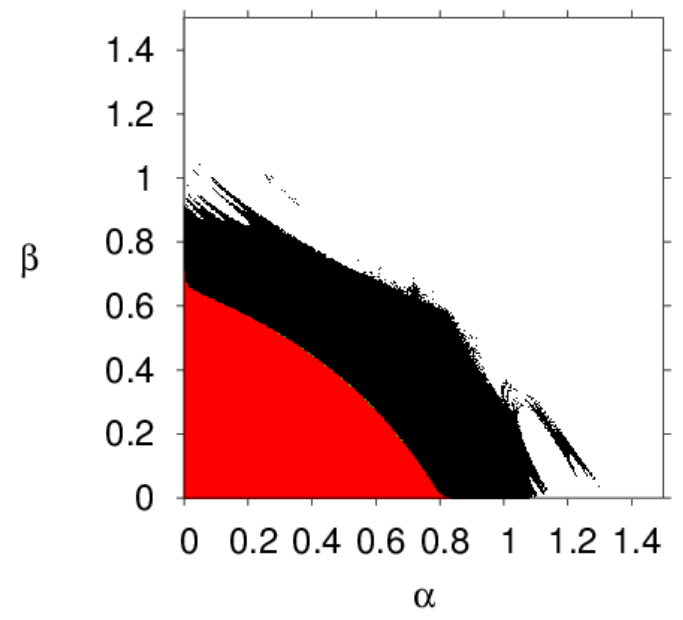

(d) $w_{1}=w_{2}=0.2$

Fig.8. Parameter basin with respect to $(\alpha, \beta)$ under different delay cases when $v=0.5, \delta=$

$$
\theta=0.8 \text {. }
$$

To have a better understanding of the impact of the adjustment speed and delay parameters on the dynamic motions of the system (24). The parameter basin, which is also called 2D bifurcation diagram is proposed to describe the complex dynamic process of the system (24) under various parameter values.

As shown in Fig.8, the different colors in each region denote their particular behaviors, that is, red, blue, green, yellow, orange, dark red, and cyan color region represent period-1, $-2,-3,-4,-5,-$ 7, -8 stable cycle respectively. Besides, the black area refers to chaos and the white area denotes the unfeasible area, which means one of the players withdraws from the market. One can check that each dynamic process in Fig.7 is consistent with the situation in Fig. 8 for the same parameter values. Moreover, one can deduce that the system (24) can present various complicated dynamic motions by adopting different values of the price adjustment speed and delay parameters. From the above numerical simulation evidence, we can clear that the delay factor plays an important role in changing the dynamic behaviors of the system (24) because that the different weights assigned to the delay parameters may expand or narrow the stability domain of Nash equilibrium. 


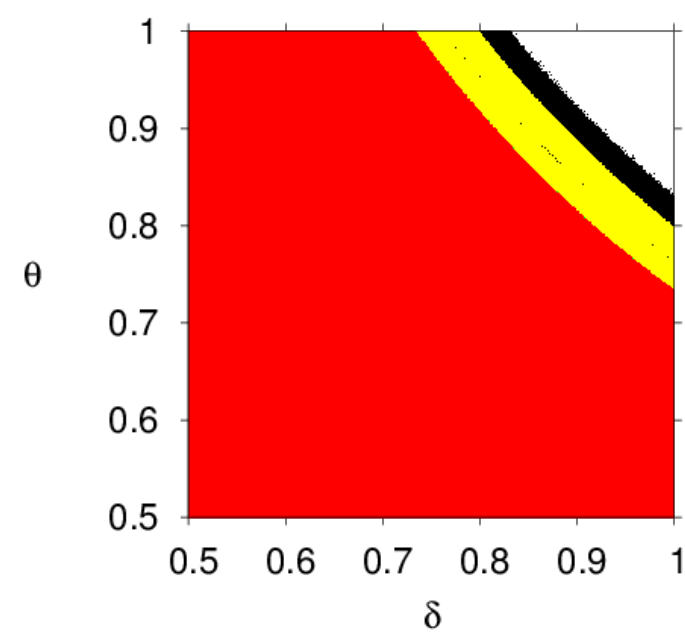

(a) $w_{1}=w_{2}=1$

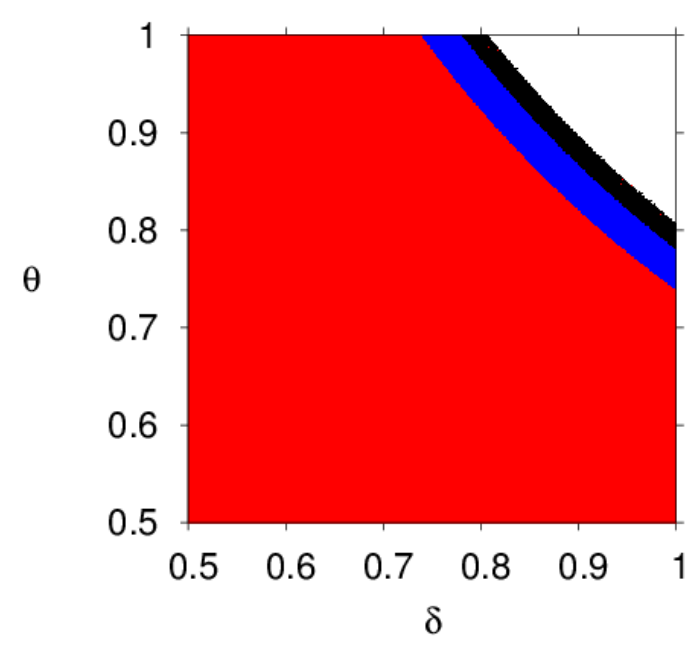

(b) $w_{1}=w_{2}=0.5$

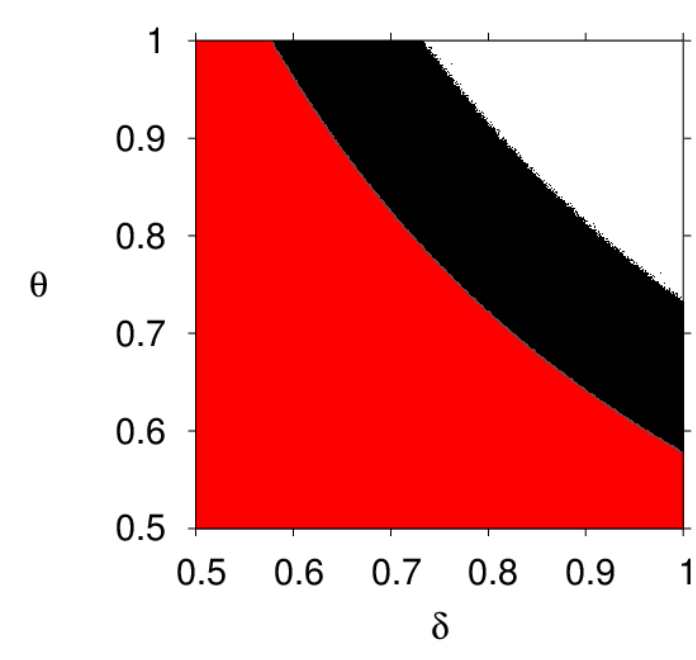

(c) $w_{1}=w_{2}=0.2$

Fig.9. The parameter basin with respect to $(\delta, \theta)$ under different delay weights when $\alpha=\beta=$ $v=0.5$.

6.2. The influence of $\delta$ and $\theta$ on the system's dynamical evolution

To figure out the influence of $\delta, \theta$ on the complex dynamics of the system (24), the parameter basin with respect to $(\delta, \theta)$ is performed under different values of the delay parameters in Fig.9. One can observe that the bifurcation curves are associated with different periodic cycles as $\delta$ and $\theta$ increase. As can be seen from Fig 9(a)-(c), no matter in which conditions, the excessively high value of the consumer acceptance to the direct channel $\theta$ or the consumer discount perception for remanufactured products $\delta$ can lead to the system becomes more complicated such as the periodic cycles and chaotic period. Therefore, we can conclude that a bigger value of the consumer acceptance degree to the direct channel or consumer discount perception for the remanufactured product has a strong destabilization effect on the system's dynamical evolution. 
In the real market, the decision-makers in dual-channel CLSC should take the consumer's behaviors into consideration. Otherwise, when the system loses its stability result from such factors, it is more difficult for the decision-makers to adopt the optimal solution to gain more profits.

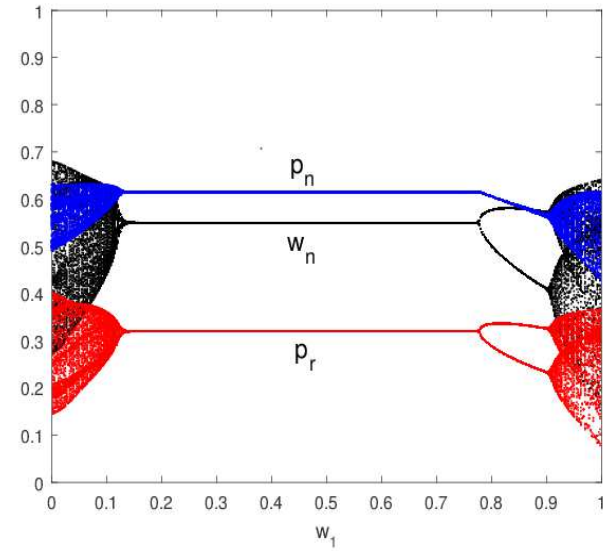

(a)

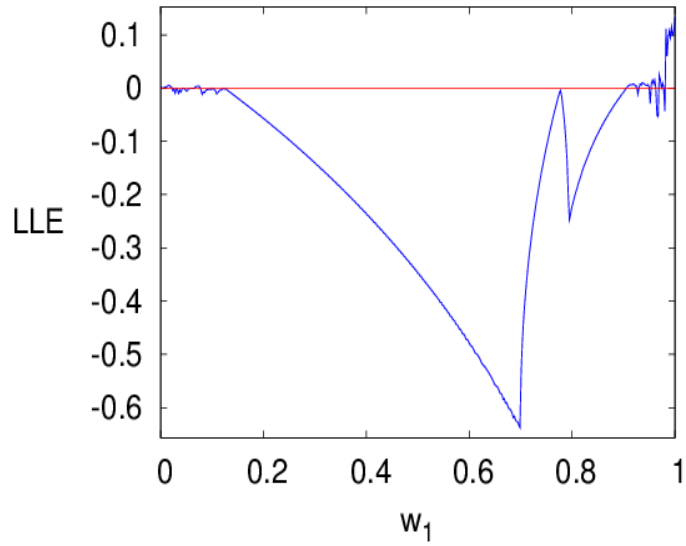

(b)

Fig.10. Bifurcation diagram and the corresponding LLEs with respect to $w_{1}$ when $\alpha=1.1, \beta=$ $0.75, v=0.5, w_{2}=1$

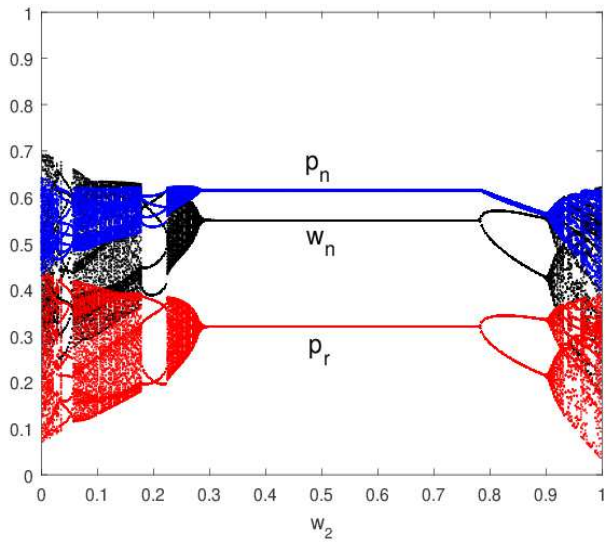

(a)

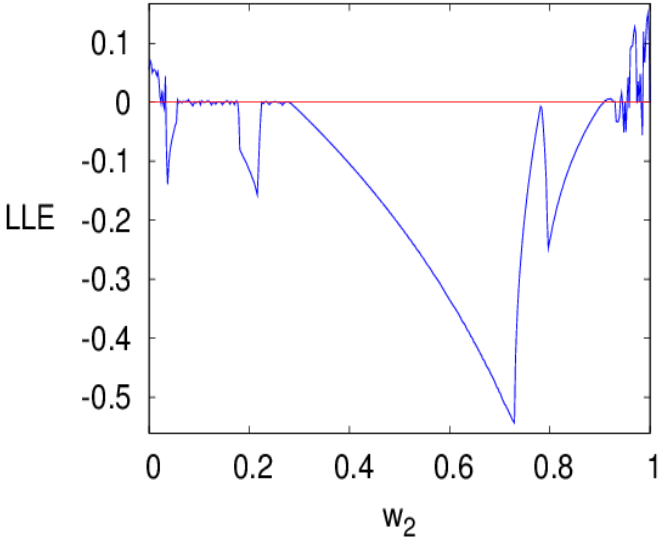

(b)

Fig.11. Bifurcation diagram and the corresponding LLEs with respect to $w_{2}$ when $\alpha=0.85, \beta=$

$$
1, v=0.5, w_{1}=1 \text {. }
$$

The impact of $w_{1}$ on the complex dynamics of the system (24) is shown in Fig.10(a) when other parameters are fixed as $\alpha=1.1, \beta=0.75, v=0.5$ and $w_{2}=1$. Under this condition, the manufacturer only uses the delay decision in the traditional channel. We can see that the price trajectories first undergoes a Neimark-Sacker bifurcation when $w_{1}$ is small enough, then it converges at Nash equilibrium point with a mild increase of $w_{1}$. As $w_{1}$ go on increasing, the Nash equilibrium point loses its stability again by experiencing a period doubling bifurcation and a 
Neimark-Sacker bifurcation, which leads to the system becomes chaotic finally. The corresponding LLE diagram in Fig.10(b) can be used to prove the above dynamic process. Furthermore, Fig.11(a) shows price bifurcation with the change of $w_{2}$ when the other parameters are fixed as $\alpha=$ $0.85, \beta=1, v=0.5, w_{1}=1$. Along with the value of $w_{2}$ varying from 0 to 1 , the behavior system (24) is very complicated, including period doubling bifurcation, stationary period, Neimark-Sacker bifurcation and chaos. The corresponding LLEs are computed as displayed in Fig.11(b) to judge the system's dynamic motion by comparing with zero.

From the above numerical experiments, the conclusion can be drawn that no matter adopting the price delay decisions in the traditional channel or the direct channel does not always make the system more stable. The manufacturer is concerned too much or too little about period $t$ price will make the system less stable. Hence, only the appropriate delay weights given to the delay factors can make the system more stable.

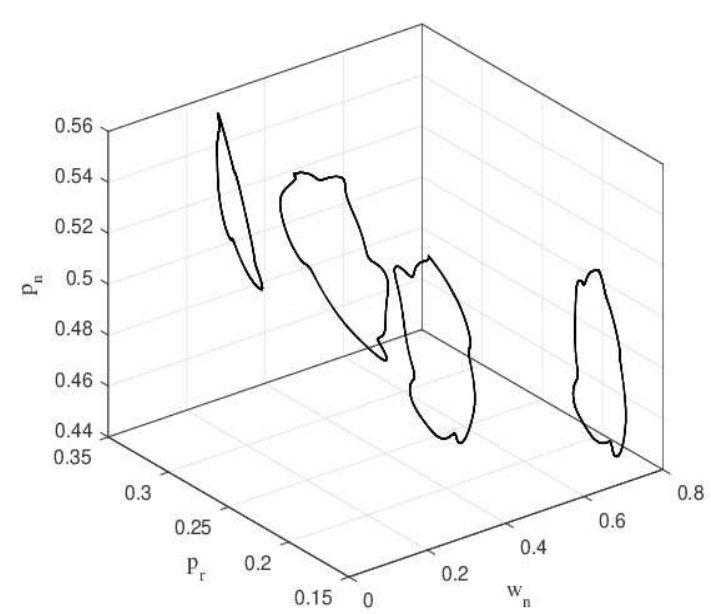

(a) $\alpha=1.45$

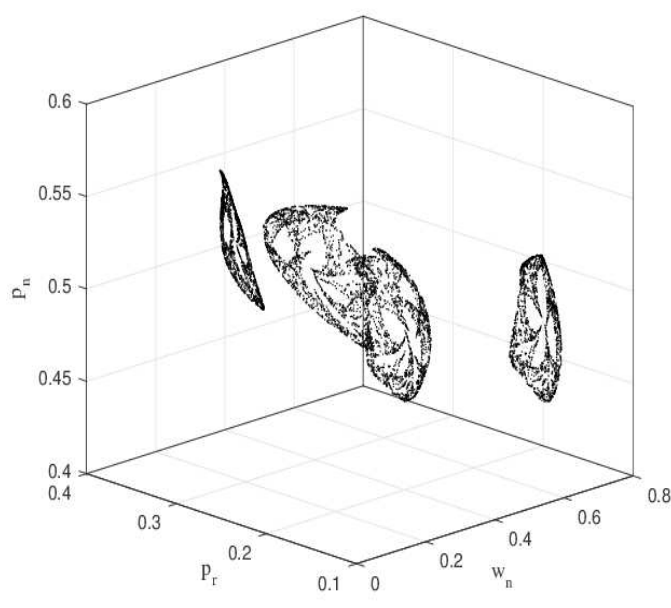

(b) $\alpha=1.48$

Fig.12. The three-dimensional limit cycles and strange attractors for $\left(w_{n}, p_{n}, p_{r}\right)$ with different values of $\alpha$ when $\beta=v=0.5, w_{1}=w_{2}=0.5$.

The three-dimensional limit cycles and strange attractors are shown in Fig 10 when the values $\beta=v=0.5, w_{1}=w_{2}=0.5$ are taken as well as $\alpha$ is assigned with different values. It is observed that there exist four quasi-periodic attractors associated with invariant closed curves are displayed in Fig.12(a) when $\alpha=1.45$. Also, the co-existing of multiple chaotic attractors which exhibit fractal structure related with $\alpha=1.48$ are presented in Fig.12(b). 


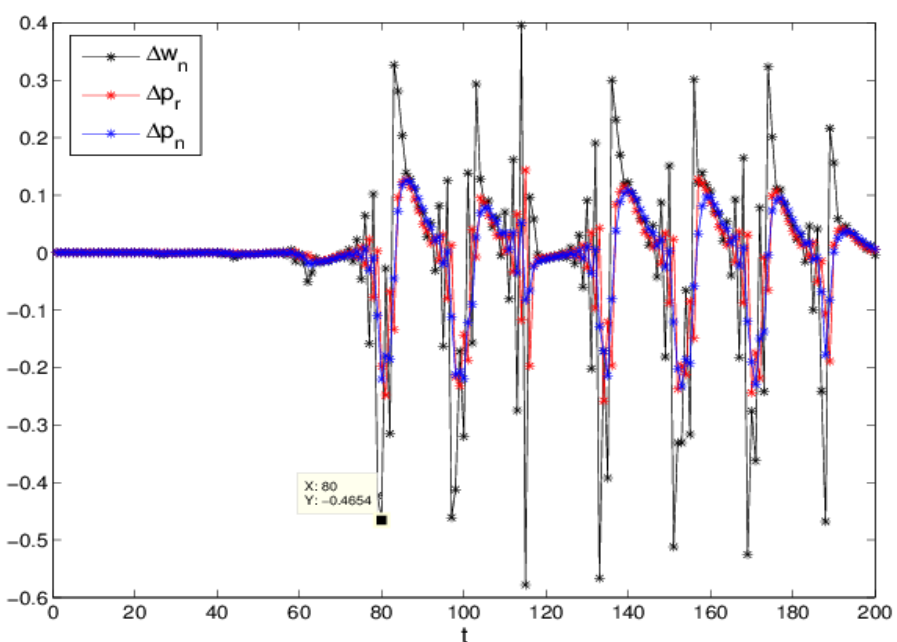

Fig.13. Time history diagram of prices with different initial conditions between $\left(w_{n}, p_{n}, p_{r}\right)=$

$$
(0.5,0.3,0.6) \text { and }\left(w_{n}, p_{n}, p_{r}\right)=(0.50001,0.3,0.6)
$$

Another important characteristic of the chaotic system is the sensitive dependence to initial conditions. Fig.13 demonstrates the difference between price trajectories with initial points $\left(w_{n}, p_{n}, p_{r}\right)=(0.5,0.3,0.6)$ and $\left(w_{n}, p_{n}, p_{r}\right)=(0.50001,0.3,0.6)$ in a chaotic period for $\alpha=$ $1.48, \beta=v=0.5, w_{1}=w_{2}=0.5$. We can see that at the beginning the two orbits are indistinguishable, but the difference between them builds up rapidly after a number of iterations (about 60 times).

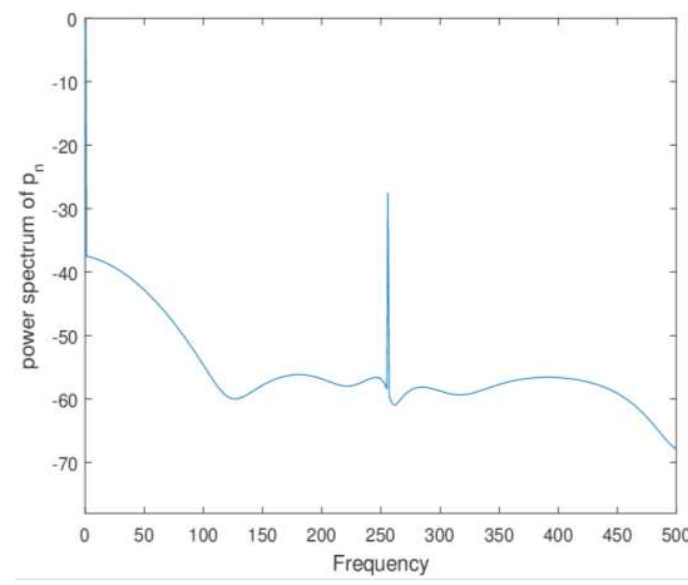

(a) $\beta=1.4($ period-4)

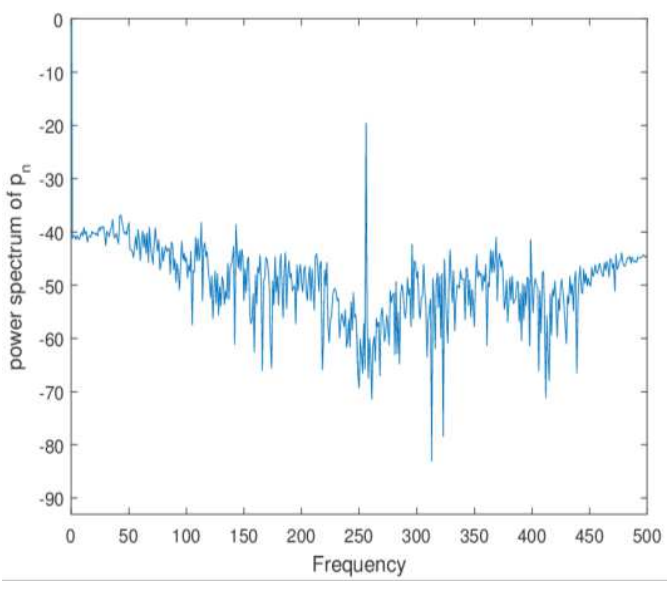

(b) $\beta=1.48($ chaos $)$

Fig.14. The power spectrum of $p_{n}$ with respect to $\beta$ when $\alpha=0.2, v=0.5$ under non-delay cases. 


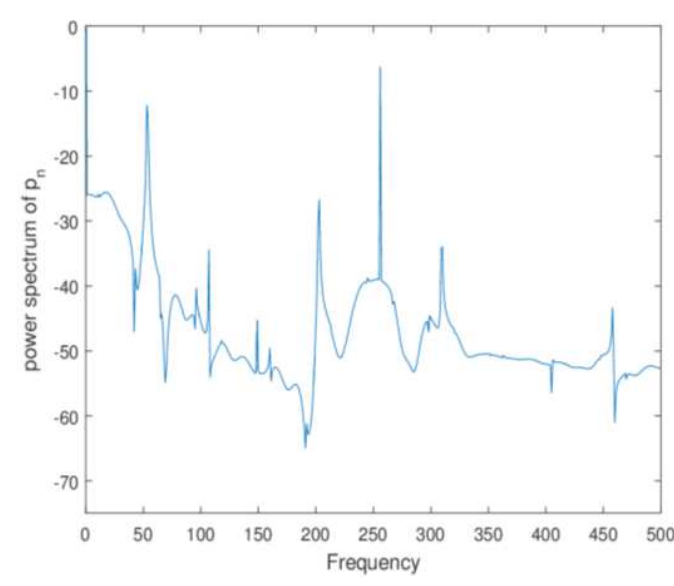

(a) $\alpha=1.44$ (quasiperiodic cycle)

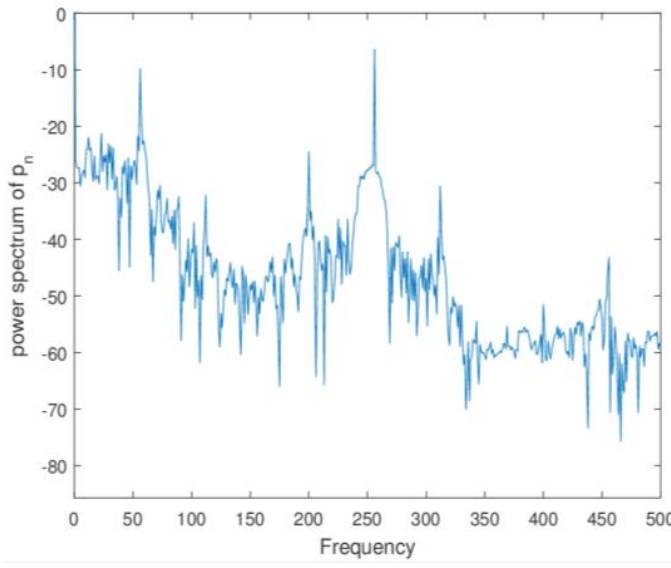

(b) $\alpha=1.48$ (chaos)

Fig.15. The power spectrum of $p_{n}$ with respect to $\alpha$ when $\beta=v=0.5, w_{1}=w_{2}=0.5$.

The power spectrum is an important approach that is widely applied in studying the system motions from a stable state to chaos. As an example, the power spectrum of $p_{n}$ with non-delay cases in terms of $\beta$ is presented by periodogram method in Fig.14. The power spectrum plotted in Fig.14(a)-(b) are associated with a periodic 4 cycle and chaotic state respectively when the other parameters are fixed as $\alpha=0.2, v=0.5$. In addition, the power spectrum of $p_{n}$ with delayed cases are plotted in Fig.15(a)-(b) which corresponding to a quasiperiodic cycle and chaotic motion with different values of $\alpha$ for $\beta=v=0.5, w_{1}=w_{2}=0.5$. One can observe that when the system is in a chaotic state, the corresponding power spectrum is characterized by white noise background and wide peaks within a limited range, and the periodic motions correspond to the peak that appears in the power spectrum.

\section{Performance measures}

In order to measure the influence of some key parameters on the channel members' performance in the dynamic Stackelberg game models. The average profit and profit level as the indexes to measure the performance of the chain members, which are frequently utilized in the business literature $[52,55]$. 


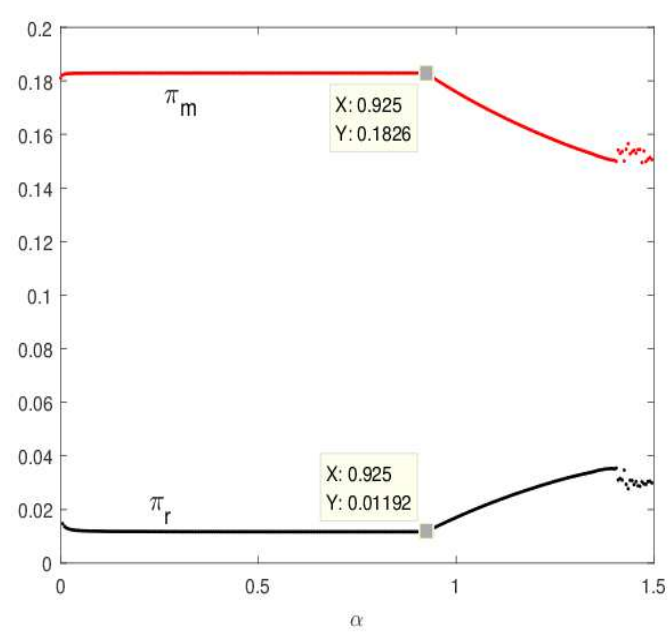

(a) $\beta=v=0.5$

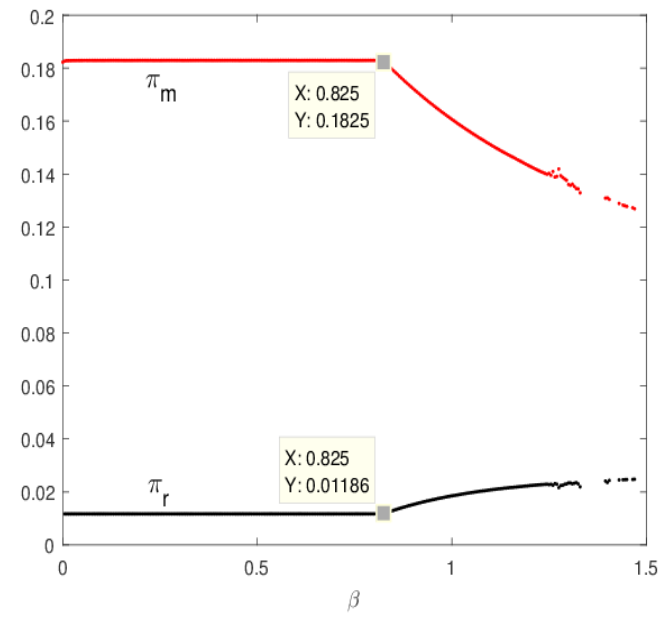

(b) $\alpha=v=0.5$

Fig.16. The average profits of the chain members with respect to the adjustment speed parameter without delay decision.

7.1 The effect of key parameters on the average profit of channel members

Fig.16 shows the average profits of the decision-makers with the changing of the adjustment speed parameter. As shown in Fig.16(a), the profits of the manufacturer and retailer are respectively fixed at their positive constants when $\alpha<0.925$, which related with the stationary period. However, when the value of $\alpha$ exceeds 0.925 , the average profit of the manufacturer trends to go down rapidly while the retailer's average profit is increased significantly with the increase of $\alpha$. Moreover, the average profit variation diagram with respect to $\beta$ is plotted in Fig.16(b). One can see that a relatively small $\beta$ will not change the average profits level of the channel members, but a sufficient bigger enough $\beta(\beta>0.825)$ can lead to the system enters into periodic and chaotic motions. Under such circumstances, the profit of the manufacturer has dropped greatly and the profit of the retailer has elevated significantly. Also, both the channel members' profits fluctuate fiercely when the system is in the chaotic period.

Thus, the conclusion can be drawn that the manufacturer can earn more profits in a stable system than that in a chaotic system. Conversely, the retailer's profit obtained in a stable system is less than that in a chaotic system. Moreover, the manufacturer's profit level is always higher than that of the retailer, but an excessive adjustment speed can reduce the profits gap between them. Naturally, we know that the manufacturer adjusts his price too fast is disadvantage to himself while the retailer can benefit from it.

Therefore, as the Stackelberg game leader, the manufacturer should be more cautious about 
updating his price strategy in each discrete time period avoid pushing the system falls into chaos.

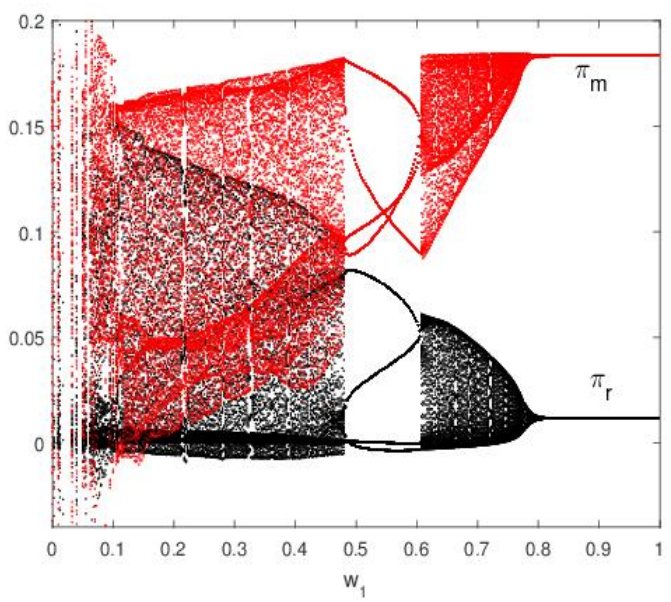

(a)

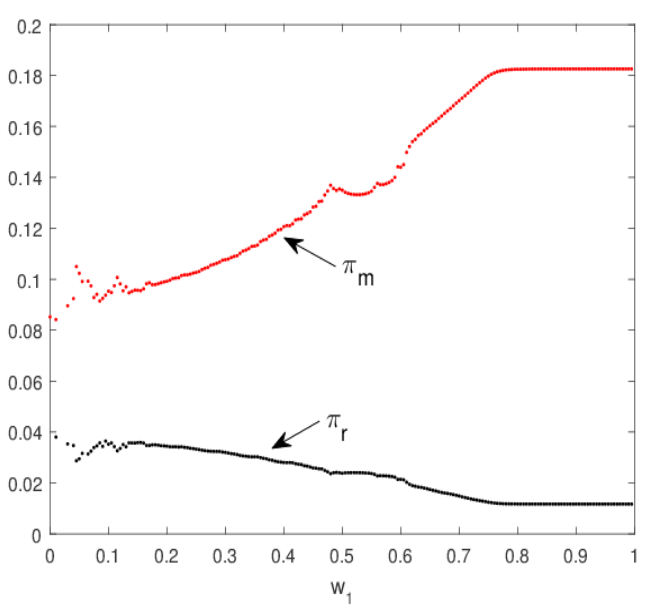

(b)

Fig.17. The profits and average profits of the chain members when $\alpha=v=0.5, \beta=1, w_{2}=0.5$

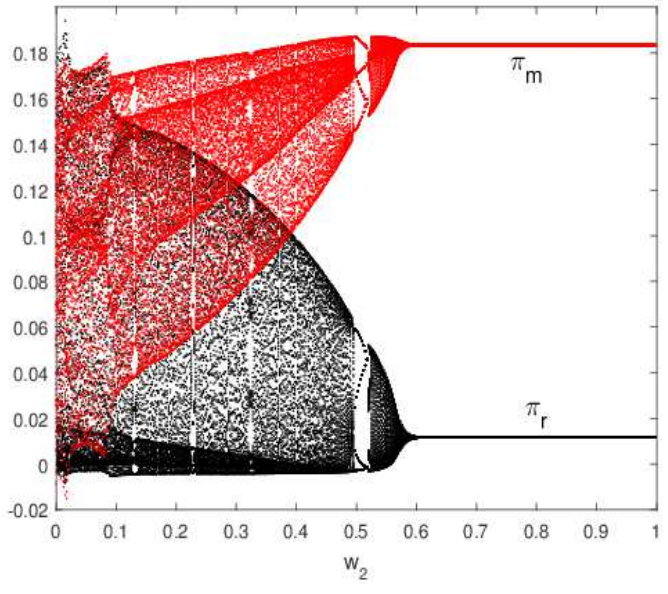

(a)

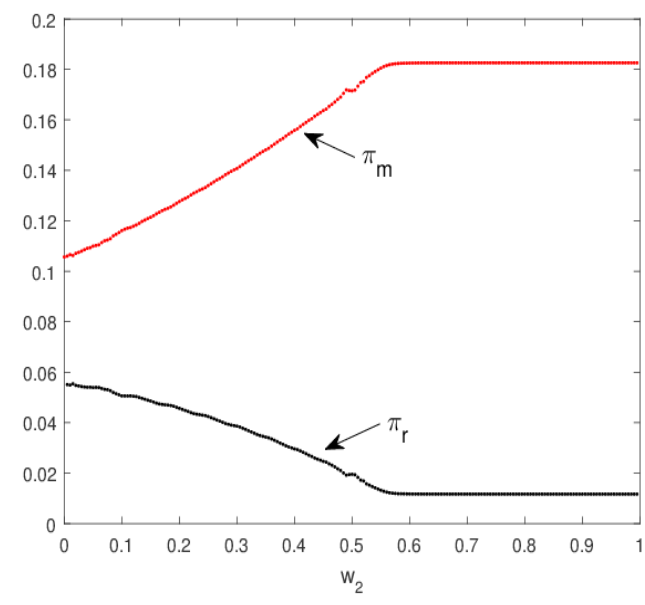

(b)

Fig.18. The profits and average profits of the chain members when $\alpha=1, \beta=v=0.5, w_{1}=0.5$

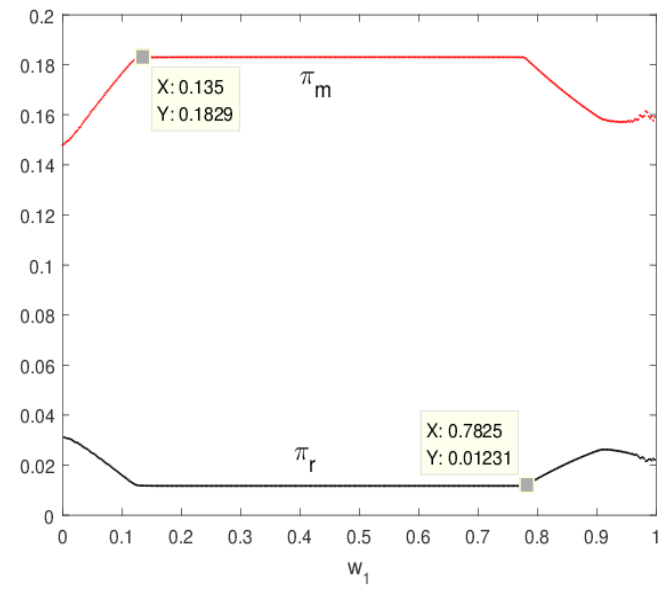

(a) $\alpha=1.1, \beta=0.75, v=0.5, w_{2}=1$

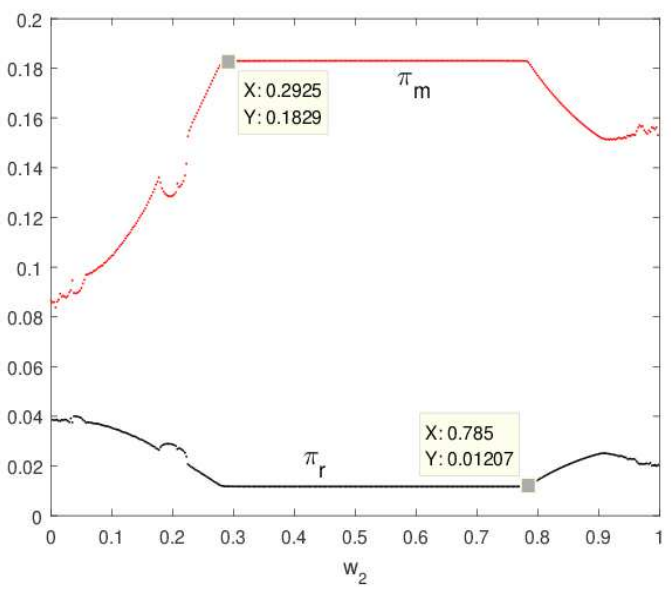

(b) $\alpha=0.85, \beta=1, v=0.5, w_{1}=1$ 
Fig.19. The average profits of the chain members with delay decisions

Fig.17-19 describes the impact of the delay factors on the performance of the channel members under various parameter values. The delay decisions are adopted both in distribution channels by the manufacturer when the system is in a period two cycle in Fig.17(a)-(b). In Fig.17(a), the channel members' profits are changed significantly with varying $w_{1}$ from 0 to 1 when the other parameters are taken as $\alpha=v=0.5, \beta=1, w_{2}=0.5$. One can observe that a small value of the delay factor $w_{1}$ can make the system much more complicated, which is conducive to the retailer capturing a higher profit while leading to the profit loss for the manufacturer. Similarly, the profits of the decision-makers with the increase of $w_{2}$ are presented in Fig.18(a)-(b). We can see that the manufacturer captures his highest profit in the stable system. Conversely, the retailer obtains her highest profit in the chaos system.

Fig.19(a) shows the average profits change with the delay decision in the traditional channel when the system is in a chaotic period. The data set we have taken is consistent with that in Fig.10(a). Consequently, the average profits change trends of the manufacturer and retailer are bound to agree with the dynamics process as shown in Fig.10(a). When a sufficient small or big delay weight given to $w_{2}$, the system will lose its stability and come into chaotic. At the same time, the manufacturer has tolerated an enormous profit loss while the retailer's profit is elevated drastically.

Fig.19(b) shows the average profit variation diagram with respect to $w_{2}$ for the same parameter values with Fig.11(a). An excessively large or small delay weight given to $w_{2}$ can't make the system more stable, but an appropriate delay weight value $\left(0.2925<w_{2}<0.785\right)$ can achieve it. Furthermore, the profits of channel members in the stable range are keeping unchanged. On the contrary, the profits which related to the chaotic range are fluctuated strongly and unpredictable.

From the above numerical analysis and simulations, some conclusions can be given as follows: the price delay decision employed by the manufacturer in the traditional channel or the direct channel can make the system more stable when an appropriate delay weight range has been adopted for the delay parameter. Therefore, implementing delay decisions is an effective measure to eliminate chaos and avoid profit loss for the manufacturer. However, being overly or much litter concern about the past period price can lead to more complex dynamic behaviors for the system (24). Also, an unsuitable delay weight will exert an adverse effect on the manufacturer's 
performance, but it is favorable for the retailer to perform a better performance. Hence, it is interesting that the chaos in the dual-channel CLSC system has a negative impact on the manufacturer while has a positive effect on the retailer. Therefore, the manufacturer is required to charge a great wealth of operational experience to apply the delay strategy properly.

7.2 Simultaneous effects of key parameters on the performance of channel members

In this subsection, in order to have a better and comprehensive knowledge of the joint effects of some key parameters, including the consumer preference to the direct channel, consumer discount perception for the remanufacturer product, the adjustment speed of price as well as the delay factors on the channel member's performance. Therefore, some numerical simulations are provided to illustrate the profit changes of the decision-makers with various parameter values.

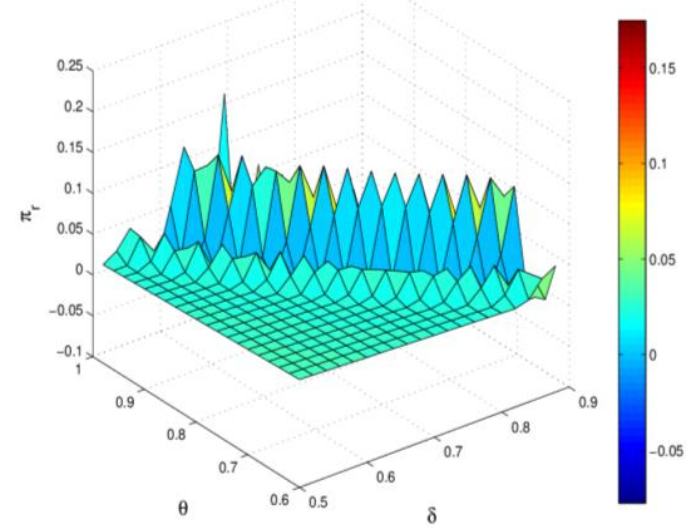

(a)

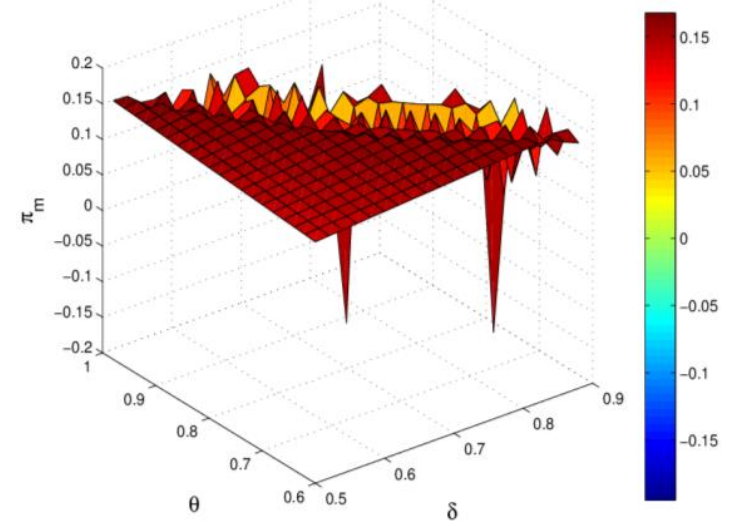

(b)

Fig.20. The influence of $\theta$ and $\delta$ on the profits of channel members when $\alpha=\beta=0.6, v=$

$$
0.5, w_{1}=w_{2}=0.2 .
$$

Firstly, we are willing to examine the simultaneous effects of the consumer preference to the direct channel $\theta$ and the consumer discount perception for the remanufactured product $\delta$ on the decision-makers' profits for $\alpha=\beta=0.6, v=0.5, w_{1}=w_{2}=0.2$. From Fig.20(a), we can see that the profit of the retailer is a slightly decreased with $\theta$ and $\delta$ increase in their small ranges. However, the higher values of $\theta$ and $\delta$ will lead to the stable system comes into a chaotic period. Correspondingly, most of the retailer's profits in the chaos range are higher than that in the stable range. In contrast, Fig.20(b) shows that the manufacturer's profit is slightly increased with $\theta$ and $\delta$ increase in their small ranges, but when the system falls into chaos result from the excessive large values of $\theta$ and $\delta$, most of his profits are changed rapidly in disorder as well as lower than that in 
the stable system.

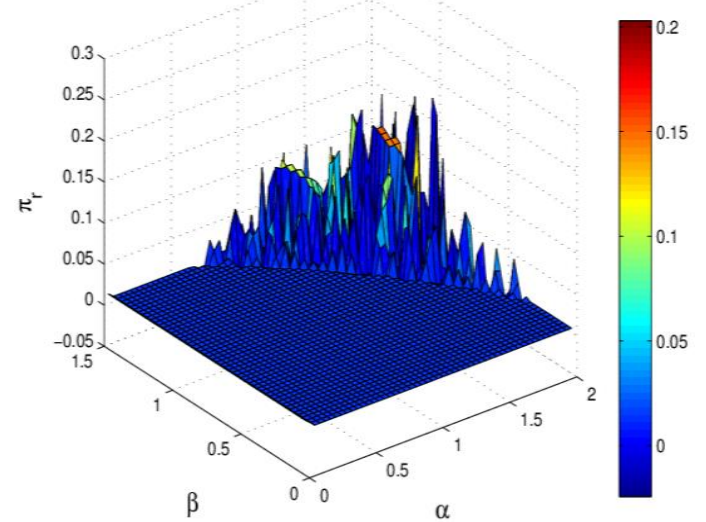

(a)

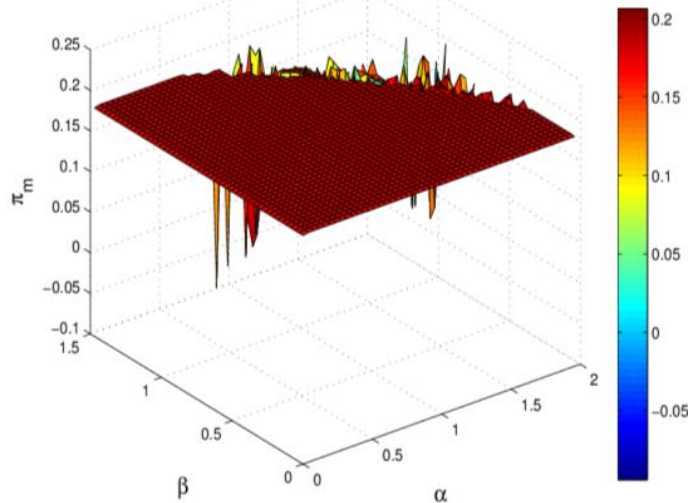

(b)

Fig.21. The influence of $\alpha$ and $\beta$ on the profits of channel members when $v=0.5, w_{1}=w_{2}=$ 0.7 .

In what follows, we want to investigate the joint influence of the price adjustment speed parameters $\alpha$ and $\beta$ on the performance of channel members as the delay weights are fixed at 0.7 . As shown in Fig.21(a), the retailer's profits are almost unchanged as $\alpha$ and $\beta$ varying in their small ranges. As the values of $\alpha$ and $\beta$ sufficiently enough will incur the system loses its stability. At that moment, the retailer's performance is immensely enhanced. On the contrary, most of the profits of the manufacturer are declined significantly with respect to the excessively high values of $\alpha$ and $\beta$ as displayed in Fig.21(b). Therefore, it is favorable for the manufacturer to employ a reasonable adjustment strategy in pricing decisions to control the system in a stable system for obtaining his highest profit.

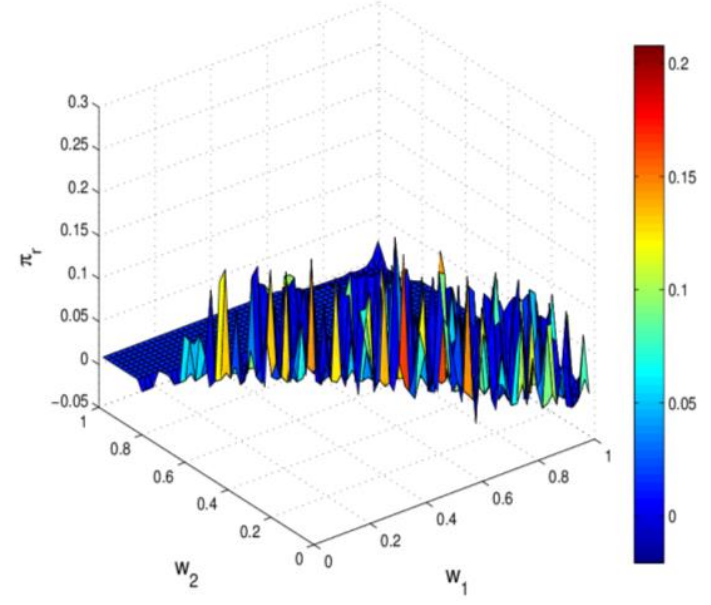

(a)

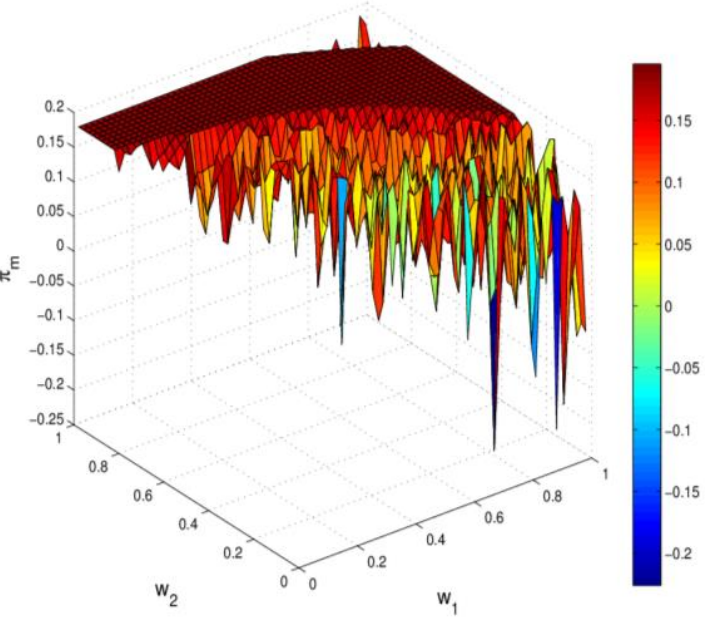

(b) 
Fig.22. The influence of $w_{1}$ and $w_{2}$ on the profits of channel members when $\alpha=0.85, \beta=$

$$
1, v=0.5
$$

Fig.22 is displayed to demonstrate the profit changes of channel members with different delay weights when the system is in a chaotic state for $\alpha=0.85, \beta=1, v=0.5$. The joint impacts of $w_{1}$ and $w_{2}$ on the profits changing of the retailer are shown in Fig.22(a). We can observe that the profits of the retailer associated with the excessively high or small delay weights are higher than that in the mild delay weights. It is because that when the manufacturer is concerned about too much or too little delay weight in the last period price will lead to the system enters into a periodic or chaotic state. The disorder market is helpful for the retailer to capture more profits. However, the changing trend of profits of the manufacturer is plotted in Fig.22(b) which opposite to that of the retailer. The manufacturer's profits have dropped rapidly related to the unfit delay weights (too small or too large weight), while an appropriate delayed decision can prompt the chaos system to returns to a stable state, which is conducive to the manufacturer improving his operating efficiency and elevating performance level.

From the above stimulating observations, we clearly know that the model parameters, viz., the consumer preference degree to the direct channel, the consumer discount perception for the remanufactured product, the price adjustment speed and the delay factors play a critical role in the dynamical evolution of the dual-channel CLSC. Also, the performance level of channel members is strongly affected by different dynamic behaviors of the system under various parameter values.

\section{Chaos control}

From the above numerical simulations, we know that the adjustment speed of price can tremendously impact the stability of system (24) and it can push the system to fall into chaos. Generally speaking, chaos is not desirable due to it will decline the players' performance. Hence, it is usually considered as a harmful factor in economic and business circumstances, which need to be controlled or eliminated. Many scholars have taken effective methods to control the system chaos under different frameworks, such as the time-delayed feedback control approach [41], variable feedback control method [57], and straight-line stabilization method [58]. In our work, the variable feedback control method is chosen to control the chaos in the dual-channel CLSC system.

To implement this control method, we need to modify the first equation of the system (24) by 
adding a variable feedback control $k w_{n}(t)$, where $k$ is the controlling coefficient. Then the controlled system can be given by:

$$
\left\{\begin{array}{c}
w_{n}(t+1)=w_{n}(t)+\alpha w_{n}(t)\left(\frac{1+2\left(w_{2} p_{r}(t)+\left(1-w_{2}\right) y(t)\right)-2\left(w_{1} w_{n}(t)+\left(1-w_{1}\right) x(t)\right)+\Delta-\delta \theta}{2(1-\delta \theta)}\right)-k w_{n}(t) \\
p_{r}(t+1)=p_{r}(t)+\beta \frac{p_{r}(t)}{2}\left(1-\frac{4\left(w_{2} p_{r}(t)+\left(1-w_{2}\right) y(t)\right)}{\delta \theta}-\frac{2\left(w_{2} p_{r}(t)+\left(1-w_{2}\right) y(t)\right)-2\left(w_{1} w_{n}(t)+\left(1-w_{1}\right) x(t)\right)+\Delta}{1-\delta \theta}\right) \\
p_{n}(t+1)=v\left(\frac{1+\left(w_{1} w_{n}(t)+\left(1-w_{1}\right) x(t)\right)+\left(w_{2} p_{r}(t)+\left(1-w_{2}\right) y(t)\right)-\delta \theta}{2}\right)+(1-v) p_{n}(t) \\
x(t+1)=w_{n}(t) \\
y(t+1)=p_{r}(t)
\end{array}\right.
$$

The chaos control process of the delayed dual-channel CLSC system is presented as below:

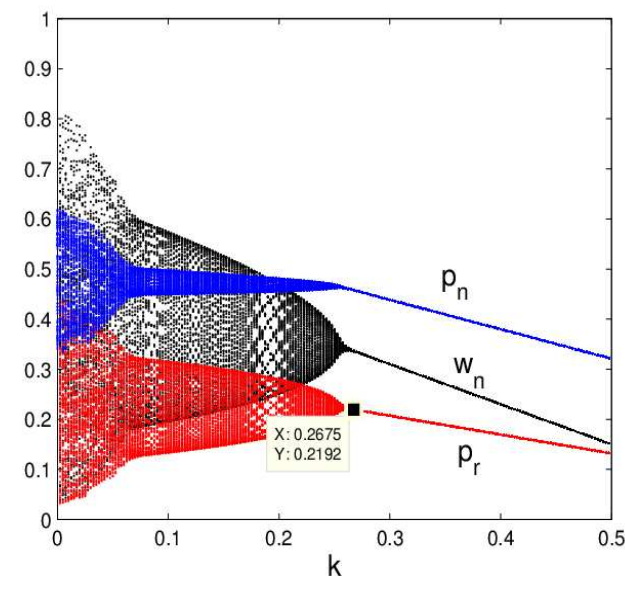

(a)

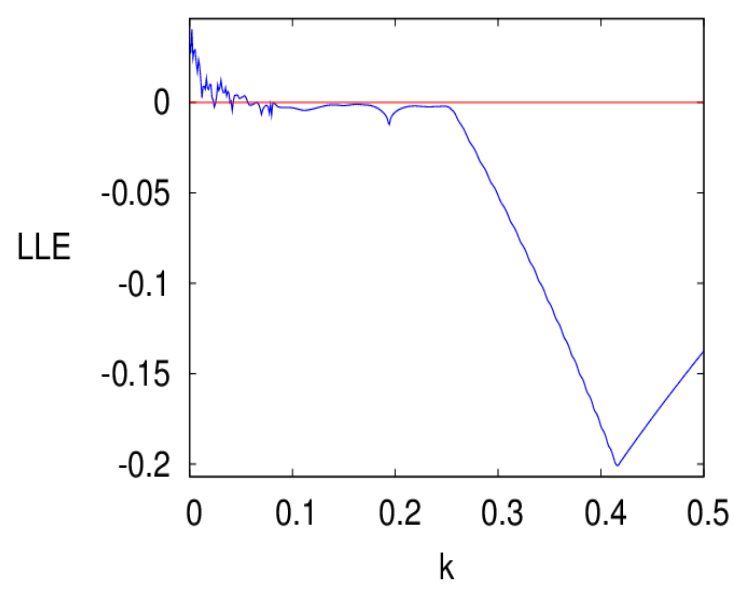

(b)

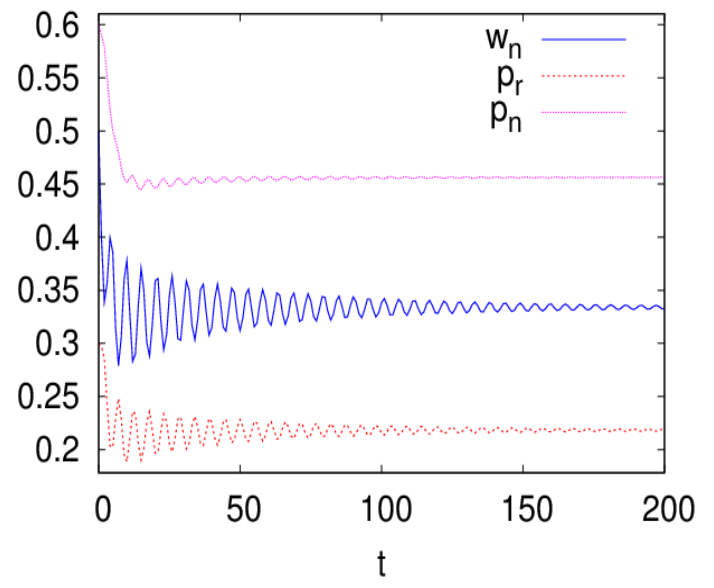

(c) $k=0.27$

Fig.23. Bifurcation and LLE diagram with respect to the controlling coefficient $k$ when $\alpha=$ $0.85, \beta=v=0.5, w_{1}=w_{2}=0.2$ (chaotic state); and the time series of price orbits with respect

$$
\text { to } k=0.27 \text {. }
$$

In Fig.23(a), we can observe that the system chaos has being gradually controlled with $k$ varying from 0 to 0.2675 . When $k>0.2675$, the system return to a stable state, which indicates the system chaos has been controlled effectively. The corresponding LLEs in Fig.23(b) are negative 
when $k>0.2675$ which can also verify the system has gotten rid of the chaos and becomes stable. Fig.23(c) shows the price orbits diagram when $k=0.27$, where one can see that the price orbits will converge to a new Nash equilibrium after a number of repeated games. It is worth remarking that the variable feedback control method would change the value of Nash equilibrium. Thus, the manufacturer has to make a trade-off between the chaos control and the best profit based on the specific condition.

\section{Conclusions}

In this paper, we mainly study two types of Stackelberg game models in a dual-channel closedloop supply chain by assuming the manufacturer and retailer adjust their prices with heterogeneous expectations, that is, the manufacturer adopts the bounded rationality expectation and the retailer employs the adaptive rule to determine their long-term pricing strategies. Moreover, the delay decision is incorporated into the pricing game model by the manufacturer to investigate its effects on the dynamic behaviors of the dual-channel CLSC system. The existence and stability of equilibrium points are analyzed. Numerical simulations are carried out to show the influence of key parameters on the complex dynamic behaviors of the dual-channel CLSC system. We obtain that the co-existing of several limit cycles and chaotic attractors may occur under some conditions, and the chaotic dynamics appear by means of flip bifurcations or Neimark-Sacker bifurcations. Moreover, we use the profit as the performance index to measure the operating efficiency of channel members under different system states. Some interesting results are derived as follows:

(1) The excessive fast adjustment speed of the bounded rational manufacturer can push the system into chaos from the stable state, while the adaptive adjustment parameter of the retailer has a very slight impact on the stability of the Nash equilibrium.

(2) The high value of the consumer discount perception for the remanufactured product or the consumer preference degree to the direct channel has a heavily destabilizing effect on the system's stability.

(3) Adopting the delay decisions by the manufacturer in his distribution channels does not always necessarily benefit for the system to stay in a stable state. The appropriate delay weight given to the past period price is conducive to preserving the system in its stability region. In contrast, the manufacturer overly or too little concerned about the price of the past period will narrow 
the stability domain and leads to the complex dynamic behaviors take place.

(4) When the system comes into periodic and chaotic motions results from the too fast adjustable speed of price or an unsuitable delay decision, which leads to the profit of the manufacturer will be declined tremendously and the retailer's profit will be increased significantly.

(5) The variable feedback control method can eliminate the delayed system chaos effectively when the value of the controlling coefficient is chosen appropriately.

Several directions for future research can be extended in the following several avenues. Firstly, model extension to incorporate the decision makers' operational behavior, such as risk aversion, fairness concern, etc., in the dual-channel CLSC. Secondly, our paper assume the market demand is determinate, so considering the uncertainty associated with the market demand is a future avenue. In addition, it will be interesting to incorporate the reverse channel selection and competition in such dual-channel CLSC in future analyses.

\section{Acknowledgements:}

This work is supported by the National Natural Science Fund of China under Grant No. 61976057, the Natural Science Fund of Shanghai under Grant No. 19ZR1417200, the Shanghai Municipal R\&D Foundation under Grant No. 20511101403, the Humanities and Social Sciences Planning Fund of Ministry of education of China under Grant No. 19YJA630116.

\section{Conflicts of Interest:}

The authors declare no conflicts of interest regarding the publication of this paper.

\section{References}

[1] Govindan, K., Soleimani, H., \& Kannan, D. (2015). Reverse logistics and closed-loop supply chain: A comprehensive review to explore the future. European Journal of Operational Research, 240(3), 603-626.

[2] Battini, D., Bogataj, M., \& Choudhary, A. (2017). Closed Loop Supply Chain (CLSC): Economics, Modelling, Management and Control. International Journal of Production Economics, 183, 319-321.

[3] Ferrer, G., \& Swaminathan, J. M. (2006). Managing New and Remanufactured Products. 
Management Science, 52(1), 15-26.

[4] Savaskan, R. C., Bhattacharya, S., \& Van Wassenhove, L. N. (2004). Closed-Loop Supply Chain Models with Product Remanufacturing. Management Science, 50(2), 239-252.

[5] Wei, J., Govindan, K., Li, Y., \& Zhao, J. (2015). Pricing and collecting decisions in a closedloop supply chain with symmetric and asymmetric information. Computers \& Operations Research, 54, 257-265.

[6] Wan, N., \& Hong, D. (2019). The impacts of subsidy policies and transfer pricing policies on the closed-loop supply chain with dual collection channels. Journal of Cleaner Production, 224, 881-891.

[7] Choi, T.-M., Li, Y., \& Xu, L. (2013). Channel leadership, performance and coordination in closed loop supply chains. International Journal of Production Economics, 146(1), 371-380.

[8] Modak, N. M., Modak, N., Panda, S., \& Sana, S. S. (2018). Analyzing structure of two-echelon closed-loop supply chain for pricing, quality and recycling management. Journal of Cleaner Production, 171, 512-528.

[9] Jalali, H., Ansaripoor, A. H., \& De Giovanni, P. (2020). Closed-loop supply chains with complementary products. International Journal of Production Economics, 229.

[10] Hong, X., Wang, Z., Wang, D., \& Zhang, H. (2013). Decision models of closed-loop supply chain with remanufacturing under hybrid dual-channel collection. The International Journal of Advanced Manufacturing Technology, 68(5-8), 1851-1865.

[11] Liu, L., Wang, Z., Xu, L., Hong, X., \& Govindan, K. (2017). Collection effort and reverse channel choices in a closed-loop supply chain. Journal of Cleaner Production, 144, 492-500.

[12] Giri, B. C., \& Dey, S. K. (2019). Game theoretic analysis of a closed-loop supply chain with backup supplier under dual channel recycling. Computers \& Industrial Engineering, 129, 179191.

[13] Huang, M., Song, M., Lee, L. H., \& Ching, W. K. (2013). Analysis for strategy of closed-loop supply chain with dual recycling channel. International Journal of Production Economics, 144(2), 510-520.

[14] Ranjbar, Y., Sahebi, H., Ashayeri, J., \& Teymouri, A. (2020). A competitive dual recycling channel in a three-level closed loop supply chain under different power structures: Pricing and collecting decisions. Journal of Cleaner Production, 272. 
[15] Yenipazarli, A. (2016). Managing new and remanufactured products to mitigate environmental damage under emissions regulation. European Journal of Operational Research, 249(1), 117130.

[16] Ferrer, G., \& Swaminathan, J. M. (2010). Managing new and differentiated remanufactured products. European Journal of Operational Research, 203(2), 370-379.

[17] Wu, C.-H. (2012). Price and service competition between new and remanufactured products in a two-echelon supply chain. International Journal of Production Economics, 140(1), 496-507.

[18] Chen, J.-M., \& Chang, C.-I. (2013). Dynamic pricing for new and remanufactured products in a closed-loop supply chain. International Journal of Production Economics, 146(1), 153-160.

[19] Lu Hsiao,Ying-Ju Chen. (2014). Strategic Motive for Introducing Internet Channels in a Supply Chain[J]. Production and Operations Management, 23(1), 36-47.

[20] Chiang W Y K, Chhajed D, Hess J D. (2003). Direct Marketing, Indirect Profits: A Strategic Analysis of Dual-Channel Supply-Chain Design[J]. Management Science, 49(1):1-20.

[21] Tsay A A, Agrawal N. Channel Conflict and Coordination in the E-Commerce Age[J]. Production and Operations Management, 2004, 13(1):93-110.

[22] Yan, R. (2008). Profit sharing and firm performance in the manufacturer-retailer dual-channel supply chain. Electronic Commerce Research, 8(3), 155-172.

[23] Dan, B., Xu, G., \& Liu, C. (2012). Pricing policies in a dual-channel supply chain with retail services[J]. International Journal of Production Economics, 139(1): 312-320.

[24] Kong, L., Liu, Z., Pan., Y., Xie, J., \&Yang, G. (2017). Pricing and service decision of dualchannel operations in an $\mathrm{O} 2 \mathrm{O}$ closed-loop supply chain. Industrial Management \& Data Systems, 117(8), 1567-1588.

[25] Xie, J., Zhang, W., Liang, L., Xia, Y., Yin, J., \& Yang, G. (2018). The revenue and cost sharing contract of pricing and servicing policies in a dual-channel closed-loop supply chain. Journal of Cleaner Production, 191, 361-383.

[26] Zheng, B., Yang, C., Yang, J., \& Zhang, M. (2017). Dual-channel closed loop supply chains: forward channel competition, power structures and coordination. International Journal of Production Research, 55(12), 3510-3527.

[27] Abbey, J. D., Meloy, M. G., Guide, V. D. R., \& Atalay, S. (2015). Remanufactured Products in Closed-Loop Supply Chains for Consumer Goods. Production and Operations Management, 
24(3), 488-503.

[28] Agrawal, V. V., Atasu, A., \& van Ittersum, K. (2015). Remanufacturing, Third-Party Competition, and Consumers' Perceived Value of New Products. Management Science, 61(1), $60-72$.

[29] Yan, W., Xiong, Y., Xiong, Z., \& Guo, N. (2015). Bricks vs. clicks: Which is better for marketing remanufactured products? European Journal of Operational Research, 242(2), 434444.

[30] Kumar Sameer, Craig Sarah. (2007). Dell, Inc.'s closed loop supply chain for computer assembly plants[J]. Information Knowledge Systems Management,6(3). 197-214.

[31] Souza, G.C., (2009). Remanufacturing in closed-loop supply chains. Production Inventory Management Journal. 45 (1), 56-66.

[32] Atalay Atasu, V. Daniel R. Guide, Luk N. Van Wassenhove. (2010). So What If Remanufacturing Cannibalizes My New Product Sales? [J]. California Management Review,52(2), 56-76.

[33] Xu, H., Liu, Z. Z., \& Zhang, S. H. (2012). A strategic analysis of dual-channel supply chain design with price and delivery lead time considerations. International Journal of Production Economics, 139(2), 654-663.

[34] Jiang, C., Xu, F., \& Sheng, Z. (2010). Pricing strategy in a dual-channel and remanufacturing supply chain system. International Journal of Systems Science, 41(7), 909-921.

[35] Gan, S.-S., Pujawan, I. N., Suparno, \& Widodo, B. (2017). Pricing decision for new and remanufactured product in a closed-loop supply chain with separate sales-channel. International Journal of Production Economics, 190, 120-132.

[36] He, P., He, Y., \& Xu, H. (2019). Channel structure and pricing in a dual-channel closed-loop supply chain with government subsidy. International Journal of Production Economics, 213, 108-123.

[37] Choi, S., \& Messinger, P. R. (2016). The role of fairness in competitive supply chain relationships: An experimental study. European Journal of Operational Research, 251(3), 798813.

[38] Zhang, Y., Chen, W., \& Mi, Y. (2020). Third-party remanufacturing mode selection for competitive closed-loop supply chain based on evolutionary game theory. Journal of Cleaner 
Production, 263.

[39] Ahmed, E., Elsadany, A. A., \& Puu, T. (2015). On Bertrand duopoly game with differentiated goods. Applied Mathematics and Computation, 251, 169-179.

[40] Askar, S. S., \& Elettreby, M. F. (2017). The impact of cost uncertainty on Cournot oligopoly games. Applied Mathematics and Computation, 312, 169-176.

[41] Peng, Y., \& Lu, Q. (2015). Complex dynamics analysis for a duopoly Stackelberg game model with bounded rationality. Applied Mathematics and Computation, 271, 259-268.

[42] Askar, S. S. (2014). Complex dynamic properties of Cournot duopoly games with convex and log-concave demand function. Operations Research Letters, 42(1), 85-90.

[43] Elsadany, A. A. (2017). Dynamics of a Cournot duopoly game with bounded rationality based on relative profit maximization. Applied Mathematics and Computation, 294, 253-263.

[44] Ding, Z., Li, Q., Jiang, S., \& Wang, X. (2015). Dynamics in a Cournot investment game with heterogeneous players. Applied Mathematics and Computation, 256, 939-950.

[45] Andaluz, J., Elsadany, A. A., \& Jarne, G. (2017). Nonlinear Cournot and Bertrand-type dynamic triopoly with differentiated products and heterogeneous expectations. Mathematics and Computers in Simulation, 132, 86-99.

[46] Wang, H., \& Ma, J. (2014). Complexity analysis of a Cournot-Bertrand duopoly game with different expectations. Nonlinear Dynamics, 78(4), 2759-2768.

[47] Elabbasy, E. M., Agiza, H. N., \& Elsadany, A. A. (2009). Analysis of nonlinear triopoly game with heterogeneous players. Computers \& Mathematics with Applications, 57(3), 488-499.

[48] Elsadany, A. A., Agiza, H. N., \& Elabbasy, E. M. (2013). Complex dynamics and chaos control of heterogeneous quadropoly game. Applied Mathematics and Computation, 219(24), 1111011118.

[49] Ding, Z., Zhu, X., \& Jiang, S. (2014). Dynamical Cournot game with bounded rationality and time delay for marginal profit. Mathematics and Computers in Simulation, 100, 1-12.

[50] Elsadany, A. A. (2010). Dynamics of a delayed duopoly game with bounded rationality. Mathematical and Computer Modelling, 52(9-10), 1479-1489.

[51] Peng, J., Miao, Z., \& Peng, F. (2011). Study on a 3-dimensional game model with delayed bounded rationality. Applied Mathematics and Computation, 218(5), 1568-1576.

[52] Li, T., \& Ma, J. (2014). Complexity analysis of the dual-channel supply chain model with delay 
decision. Nonlinear Dynamics, 78(4), 2617-2626.

[53] Elsadany, A. A., \& Awad, A. M. (2016). Dynamical analysis of a delayed monopoly game with a log-concave demand function. Operations Research Letters, 44(1), 33-38.

[54] Guo, Y., \& Ma, J. (2013). Research on game model and complexity of retailer collecting and selling in closed-loop supply chain. Applied Mathematical Modelling, 37(7), 5047-5058.

[55] Ma, J., Zhang, F., \& Jiang, H. (2020). Dynamic Pricing Game Under Different Channel Power Structures in a Closed-Loop Supply Chain. International Journal of Bifurcation and Chaos, 30(04):2050052.

[56] Zhang, F., \& Ma, J. (2016). Research on the complex features about a dual-channel supply chain with a fair caring retailer. Communications in Nonlinear Science and Numerical Simulation, 30(1-3), 151-167.

[57] Ma, J., \& Xie, L. (2015). The comparison and complex analysis on dual-channel supply chain under different channel power structures and uncertain demand. Nonlinear Dynamics, 83(3), 1379-1393.

[58] Du, J.-g., Huang, T., \& Sheng, Z. (2009). Analysis of decision-making in economic chaos control. Nonlinear Analysis: Real World Applications, 10(4), 2493-2501. 
Figures

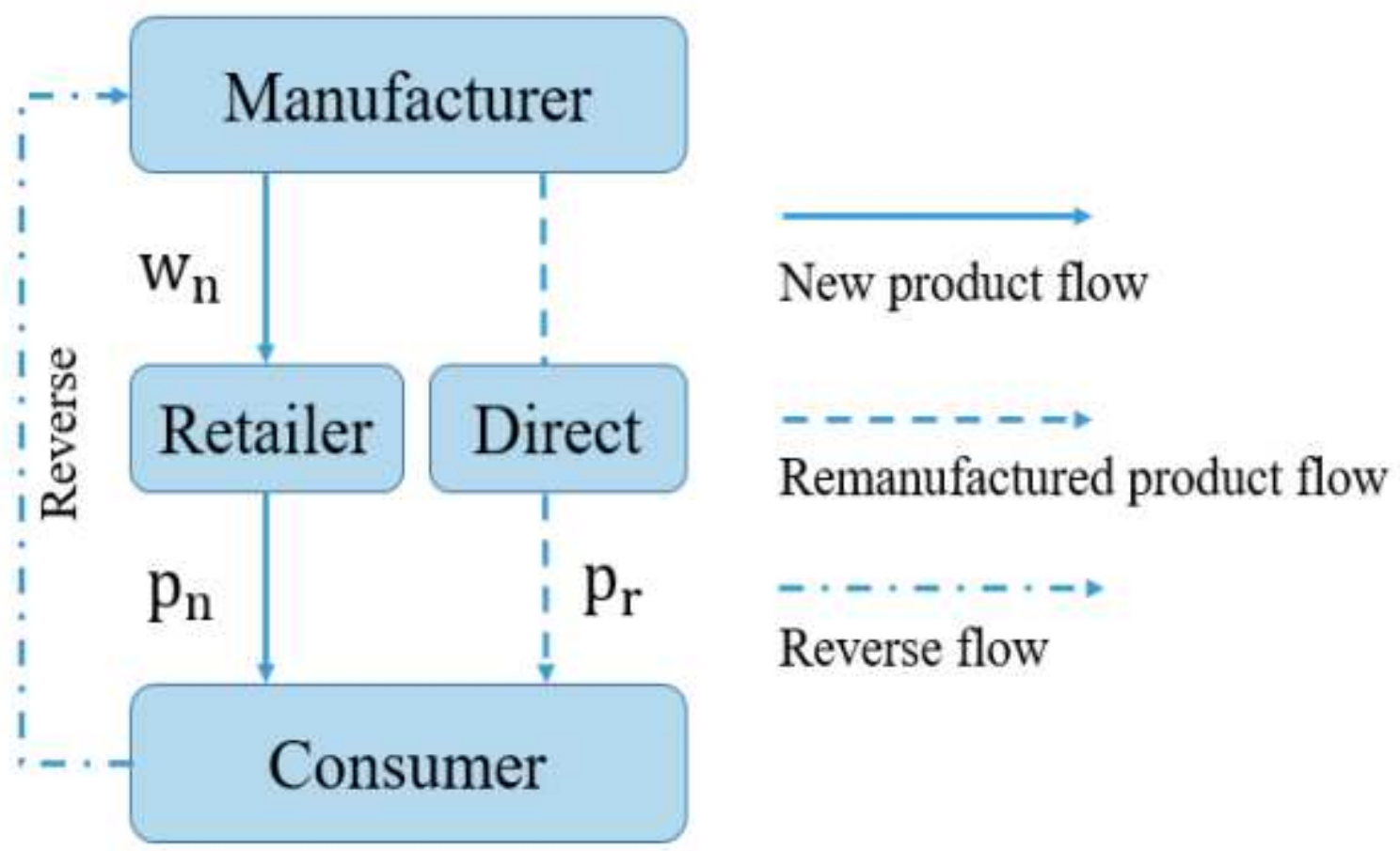

Figure 1

Please see the Manuscript PDF file for the complete figure caption 


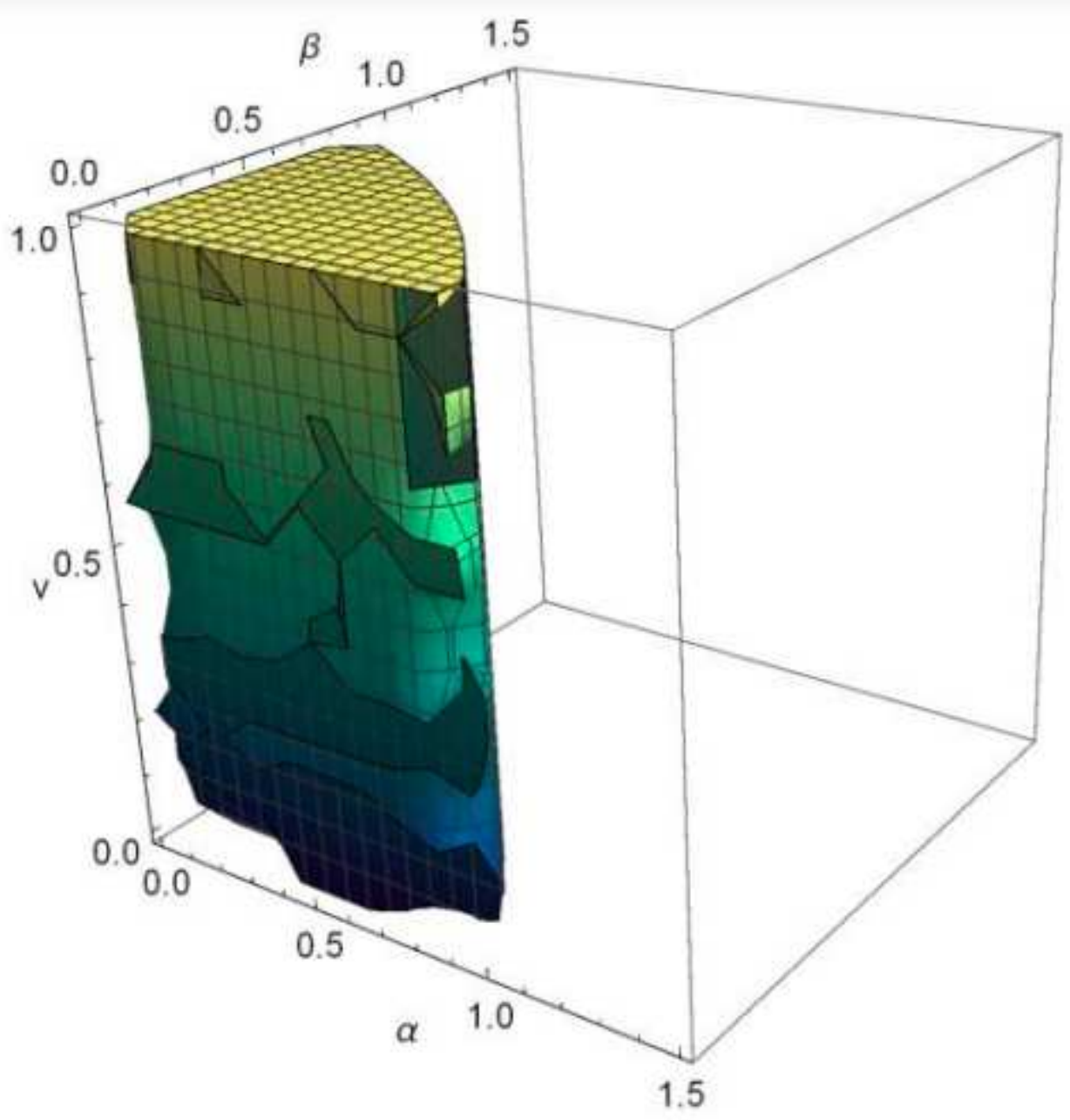

Figure 2

Please see the Manuscript PDF file for the complete figure caption

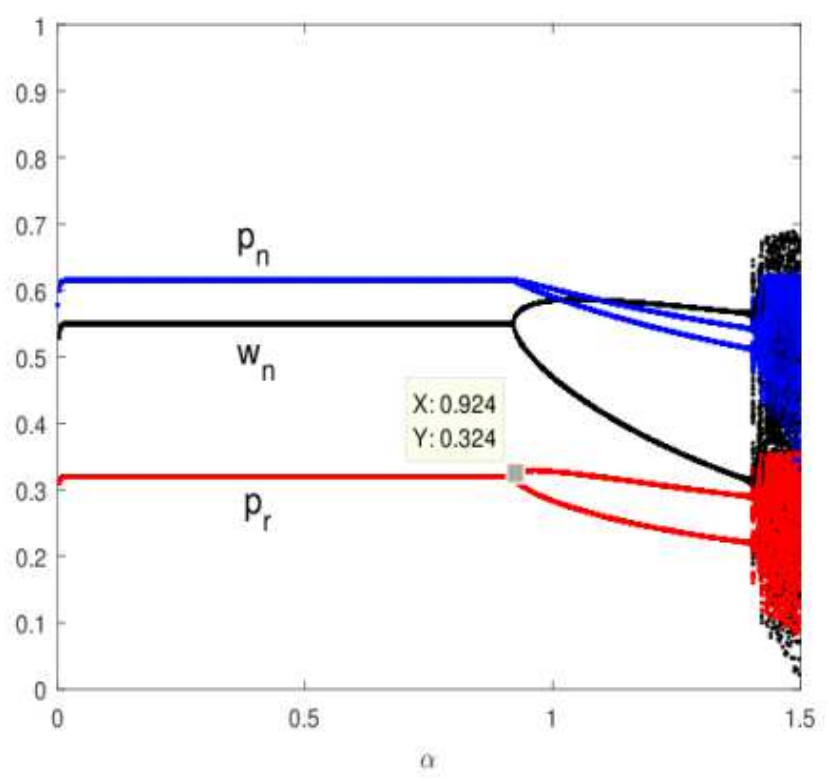

(a)

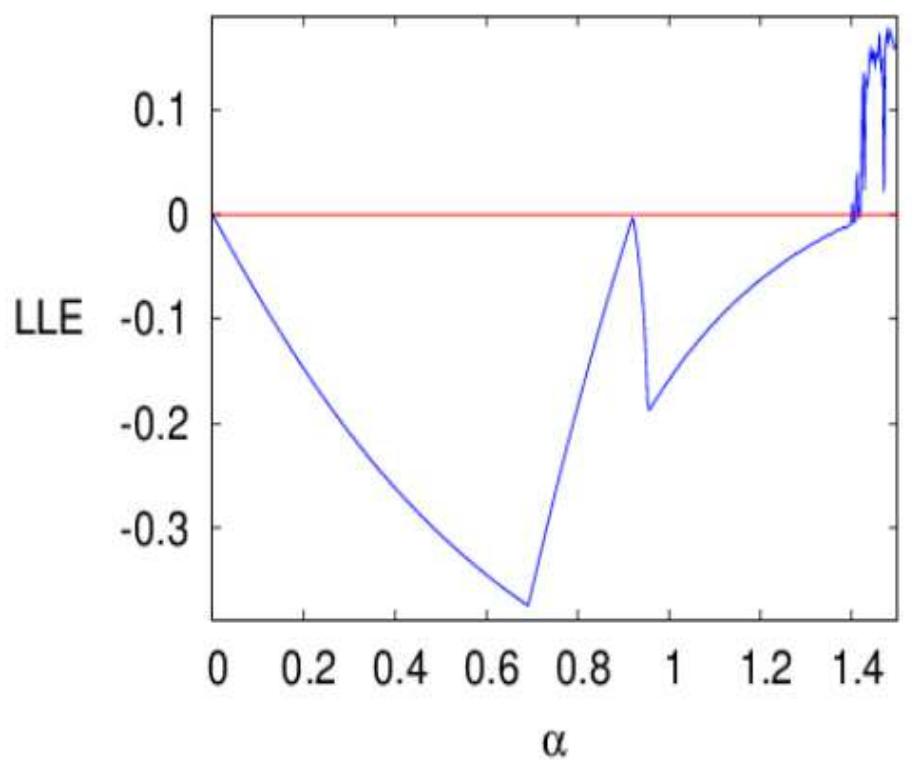

(b) 
Figure 3

Please see the Manuscript PDF file for the complete figure caption

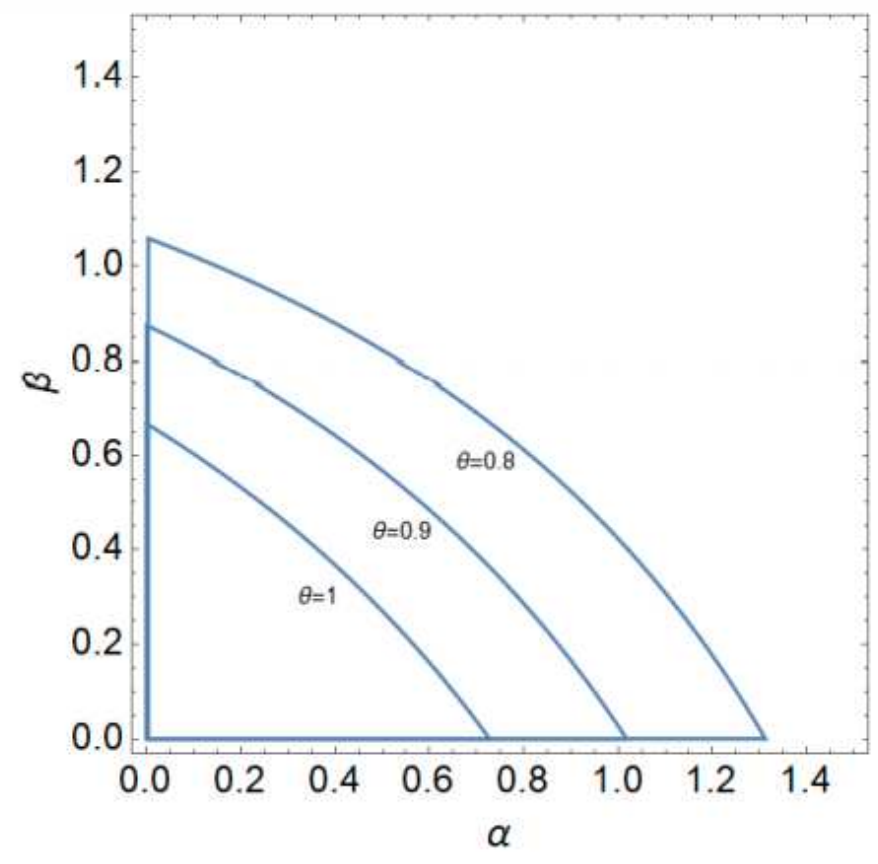

(a) $\delta=0.8, v=0.5$

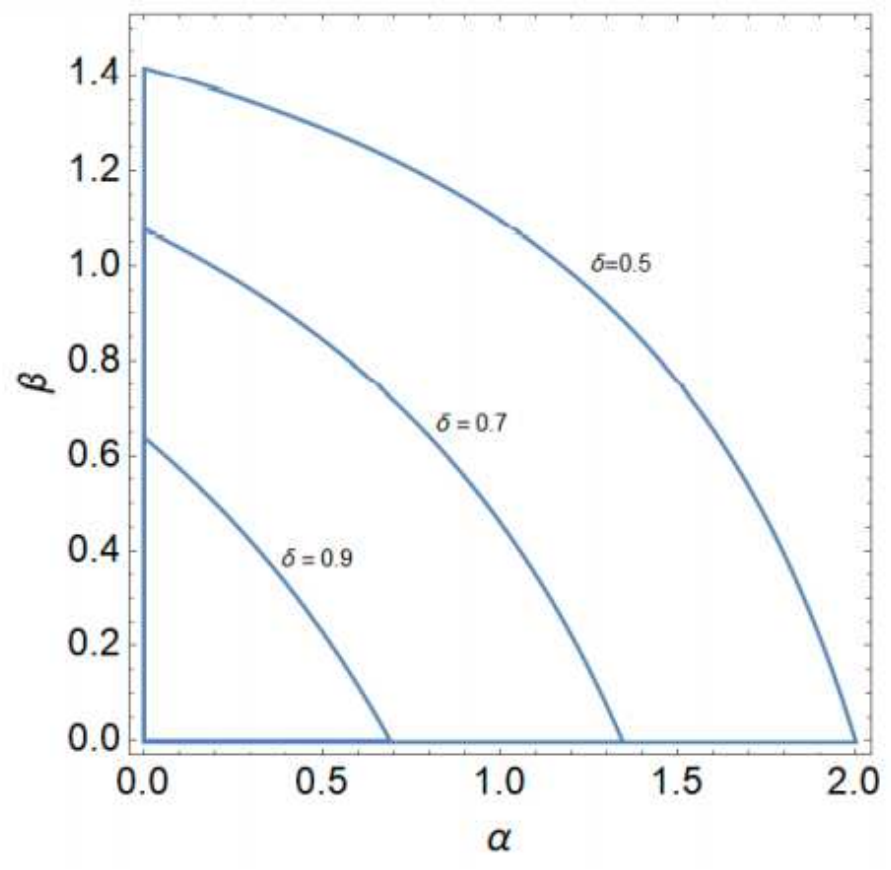

(b) $\theta=0.9, v=0.5$

\section{Figure 4}

Please see the Manuscript PDF file for the complete figure caption

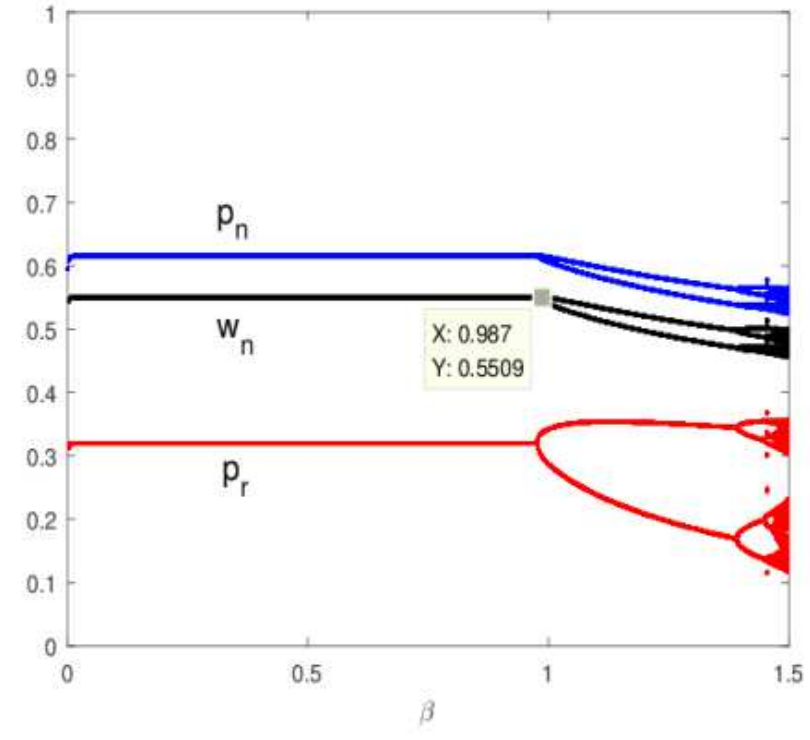

(a)

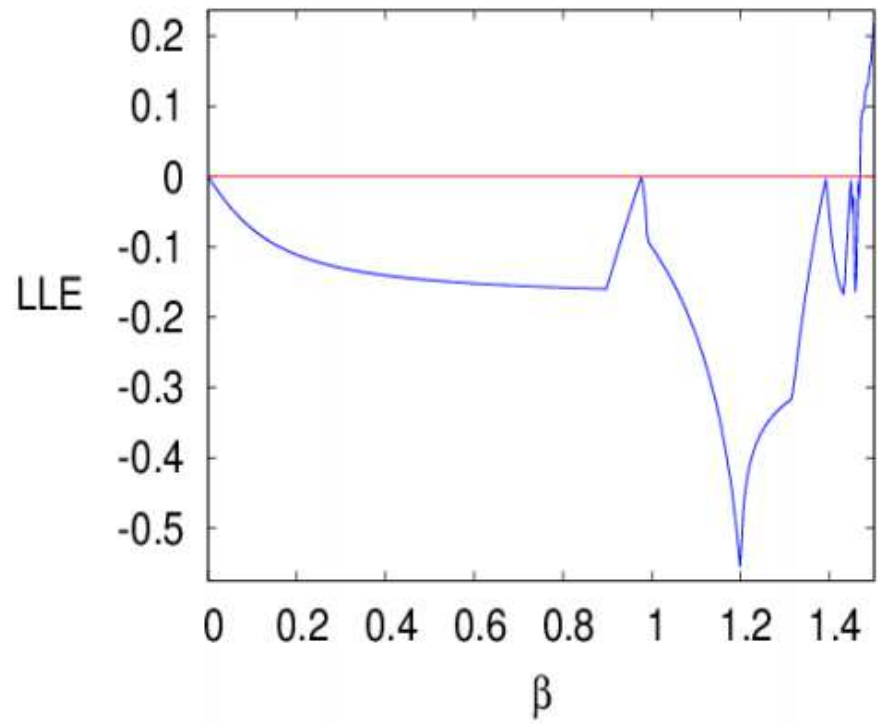

(b)

Figure 5

Please see the Manuscript PDF file for the complete figure caption 

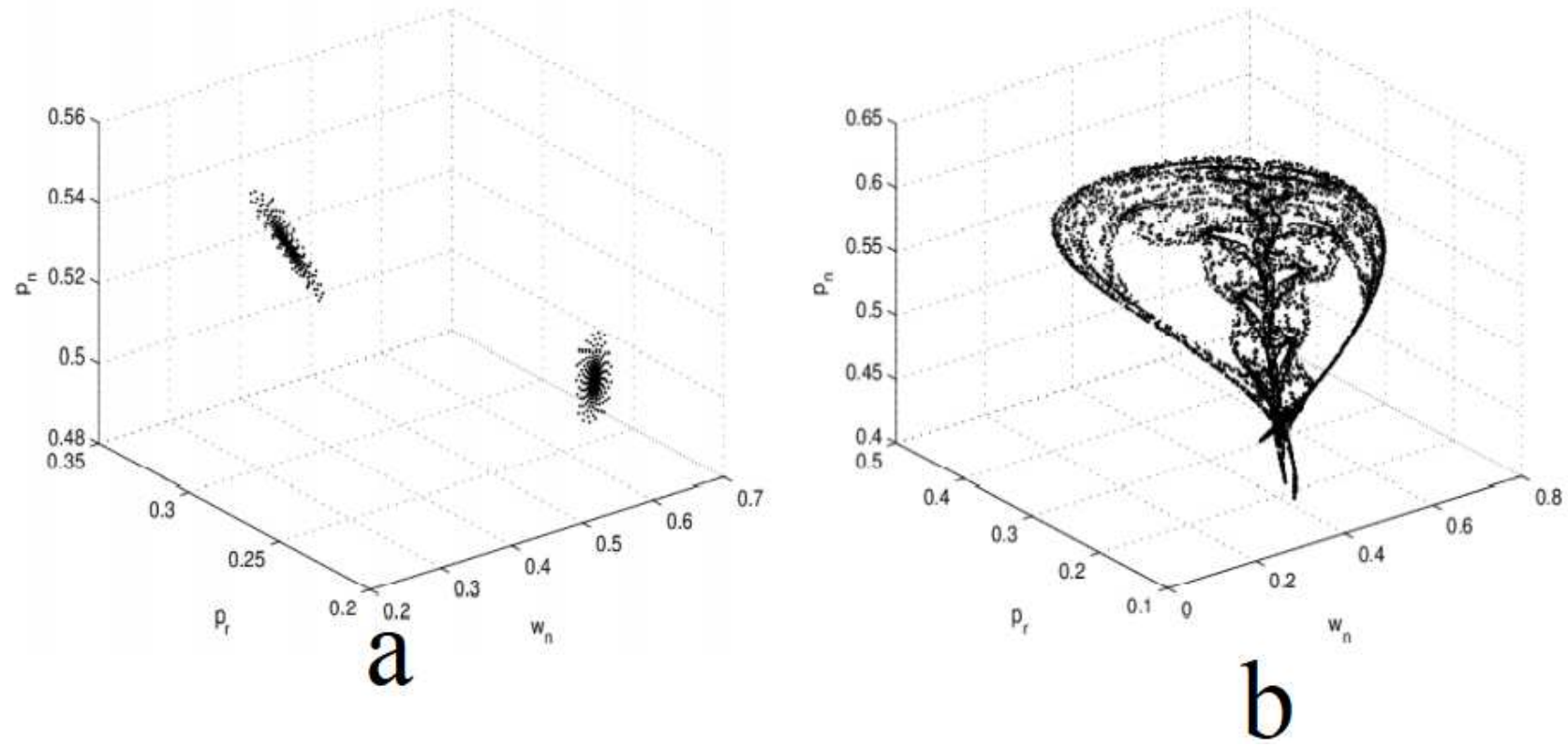

Figure 6

Please see the Manuscript PDF file for the complete figure caption 


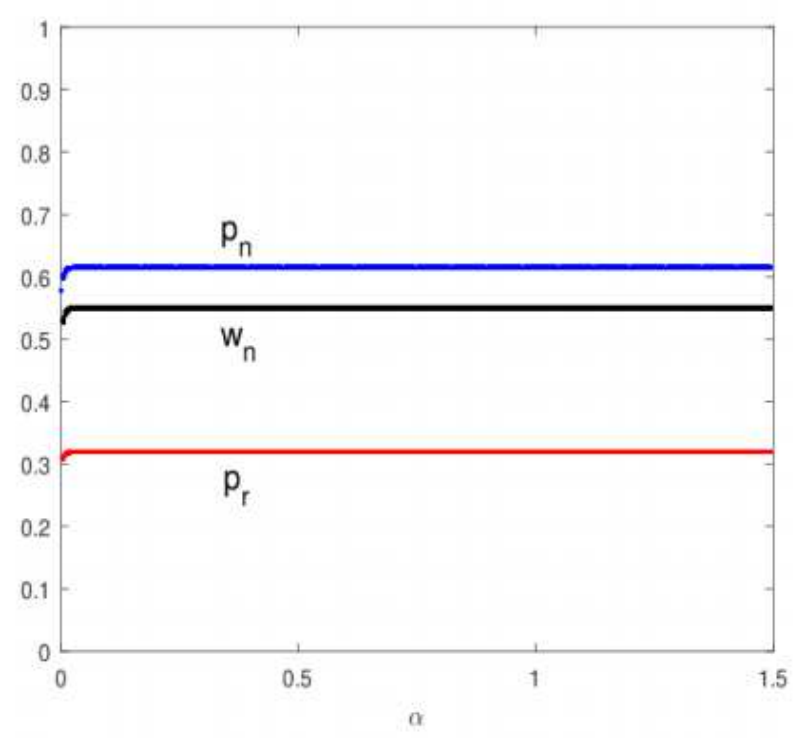

(a) $w_{1}=w_{2}=0.8$

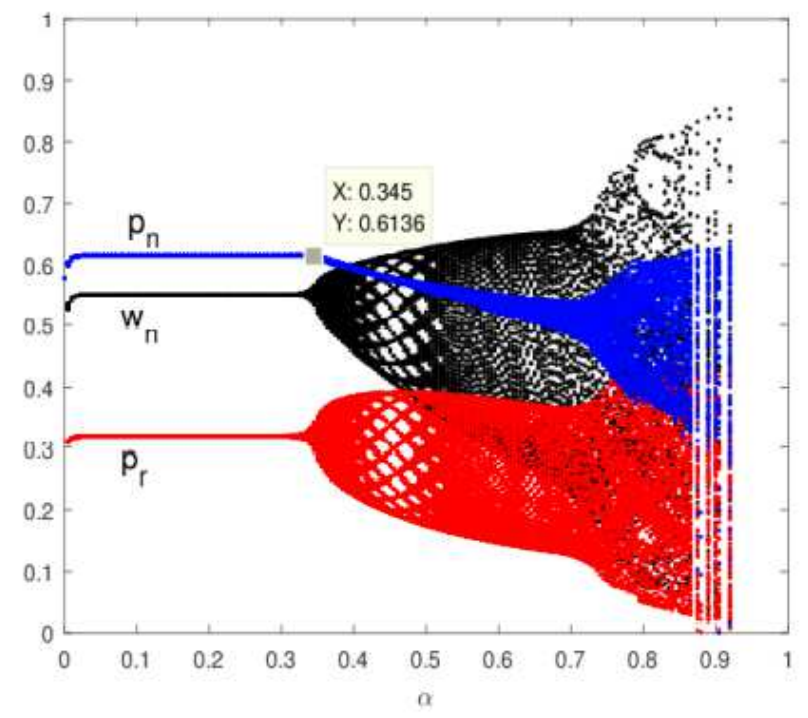

(c) $w_{1}=w_{2}=0.2$

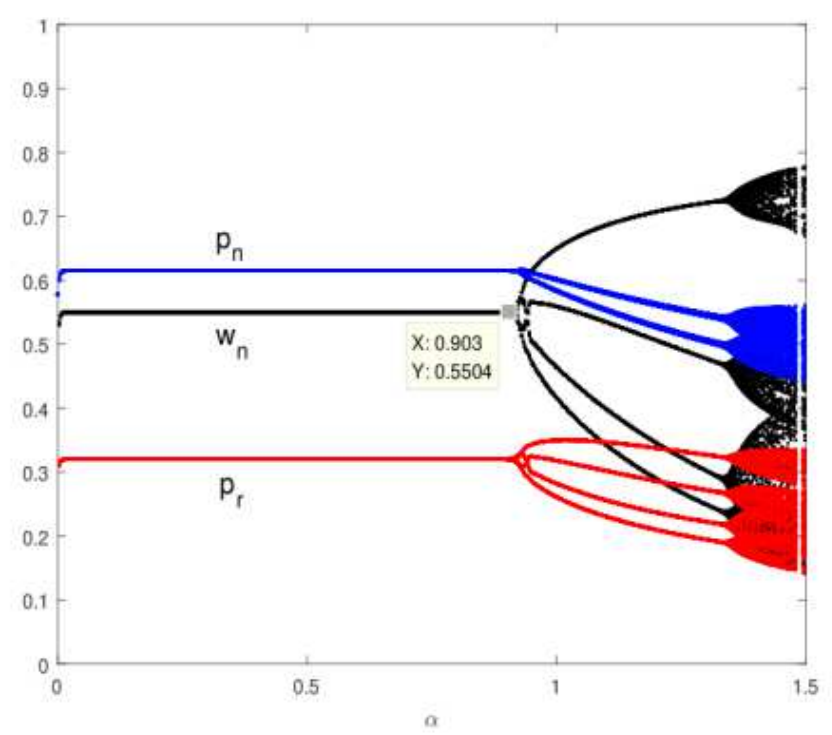

(b) $w_{1}=w_{2}=0.5$

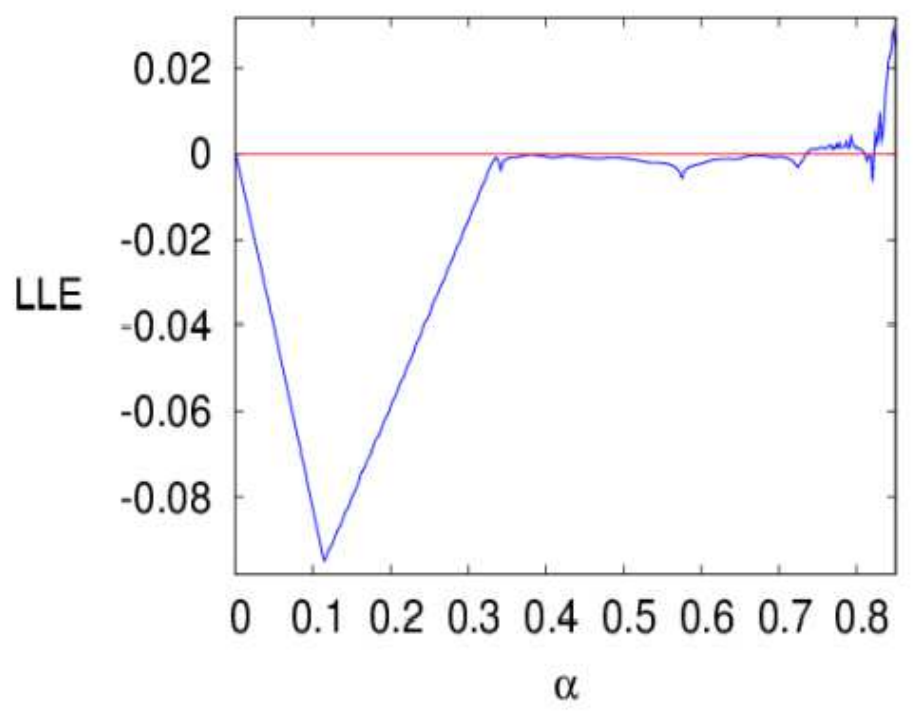

(d) $w_{1}=w_{2}=0.2$

\section{Figure 7}

Please see the Manuscript PDF file for the complete figure caption 


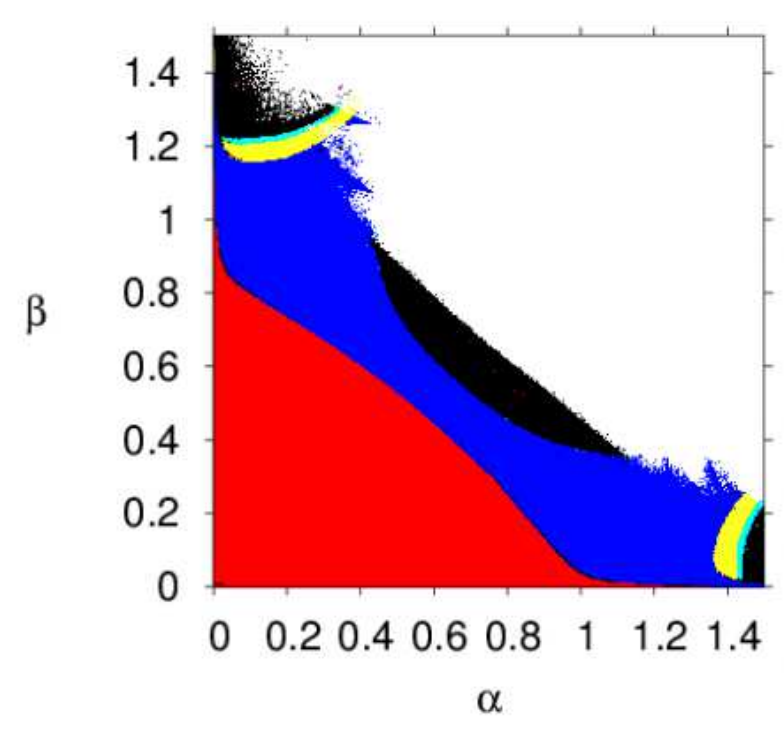

(a) $w_{1}=w_{2}=1$

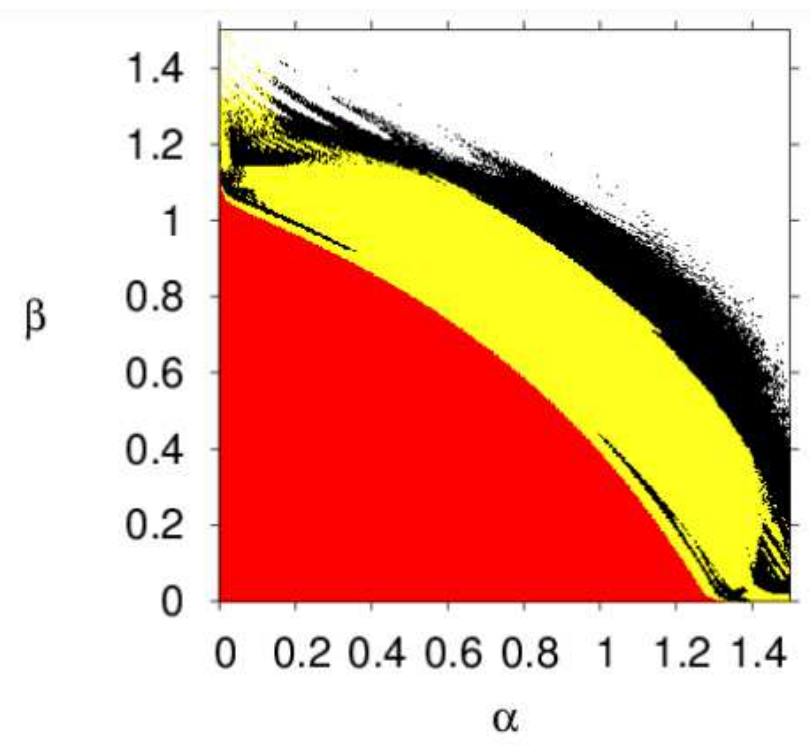

(c) $w_{1}=w_{2}=0.5$

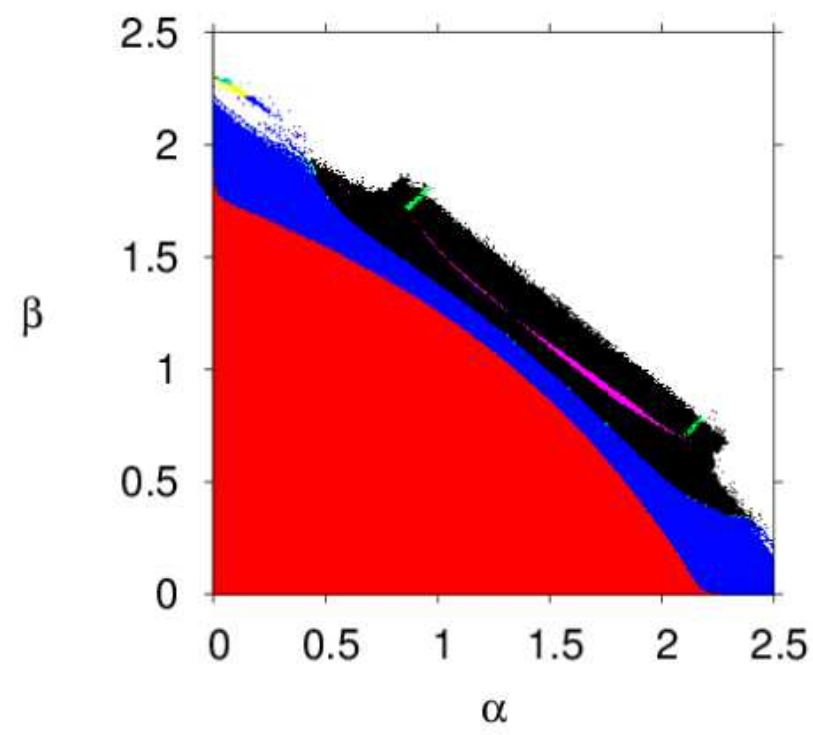

(b) $w_{1}=w_{2}=0.8$

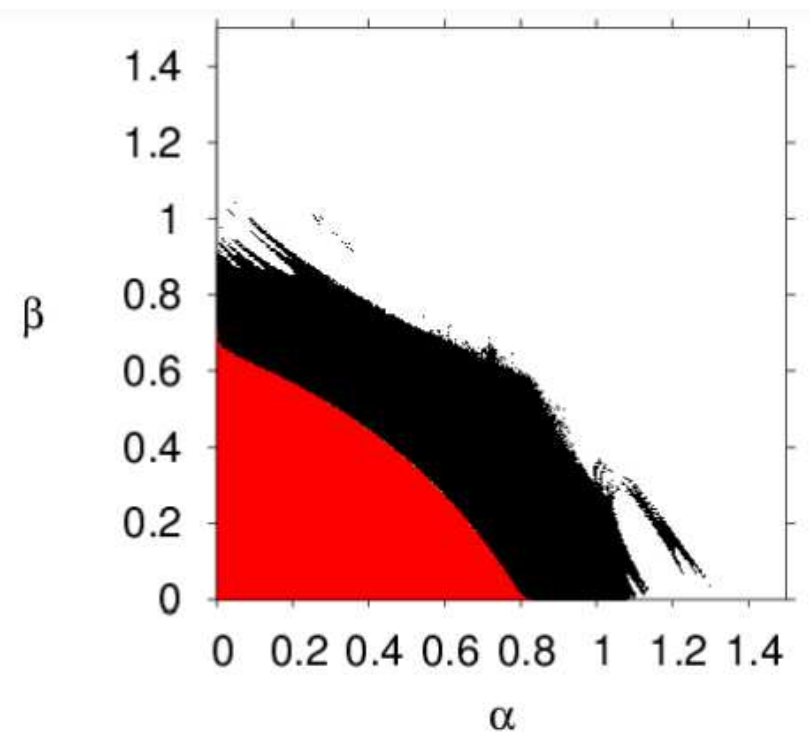

(d) $w_{1}=w_{2}=0.2$

\section{Figure 8}

Please see the Manuscript PDF file for the complete figure caption 


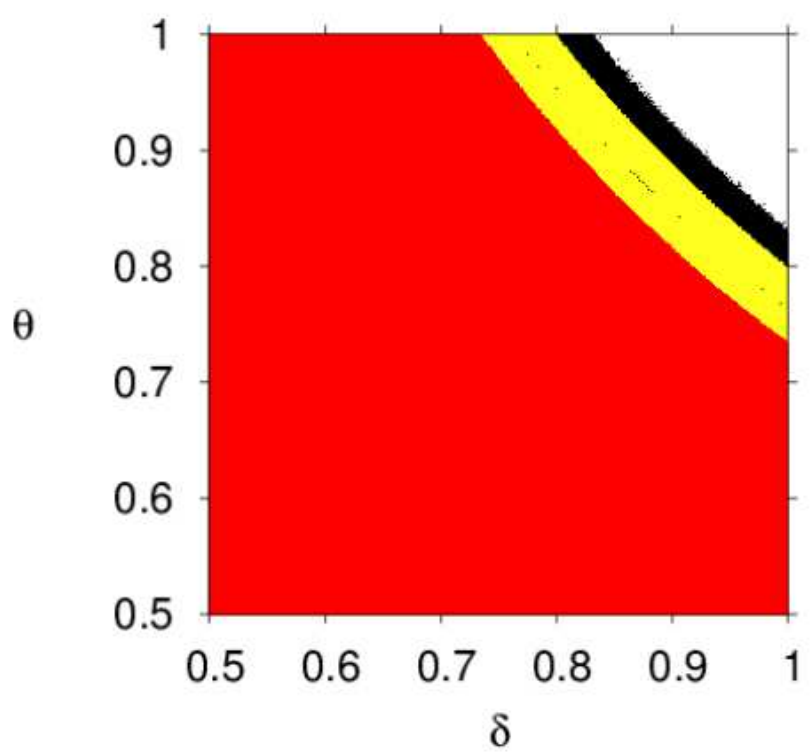

(a) $w_{1}=w_{2}=1$

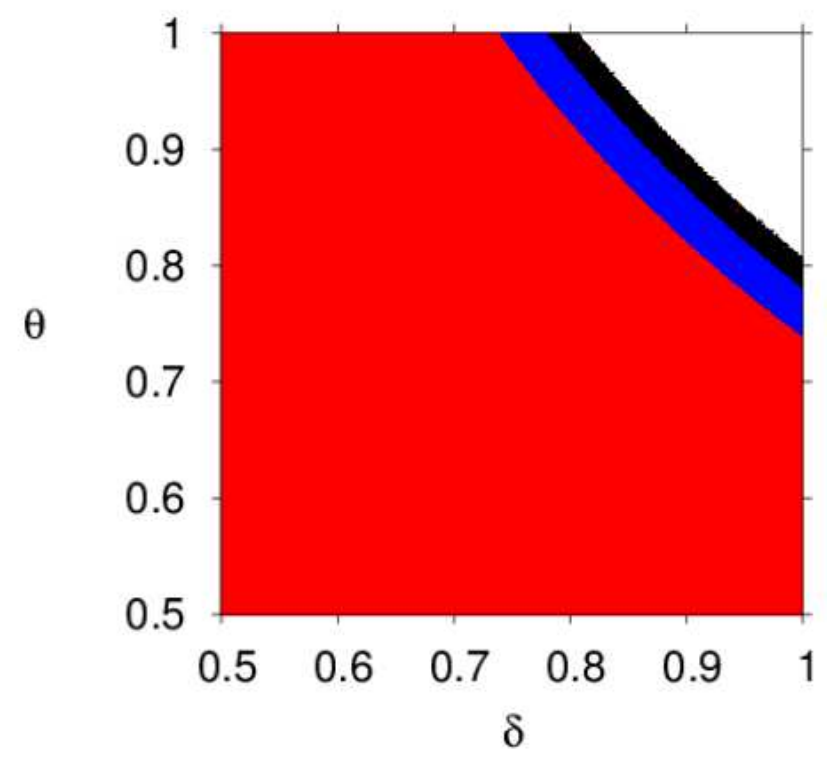

(b) $w_{1}=w_{2}=0.5$

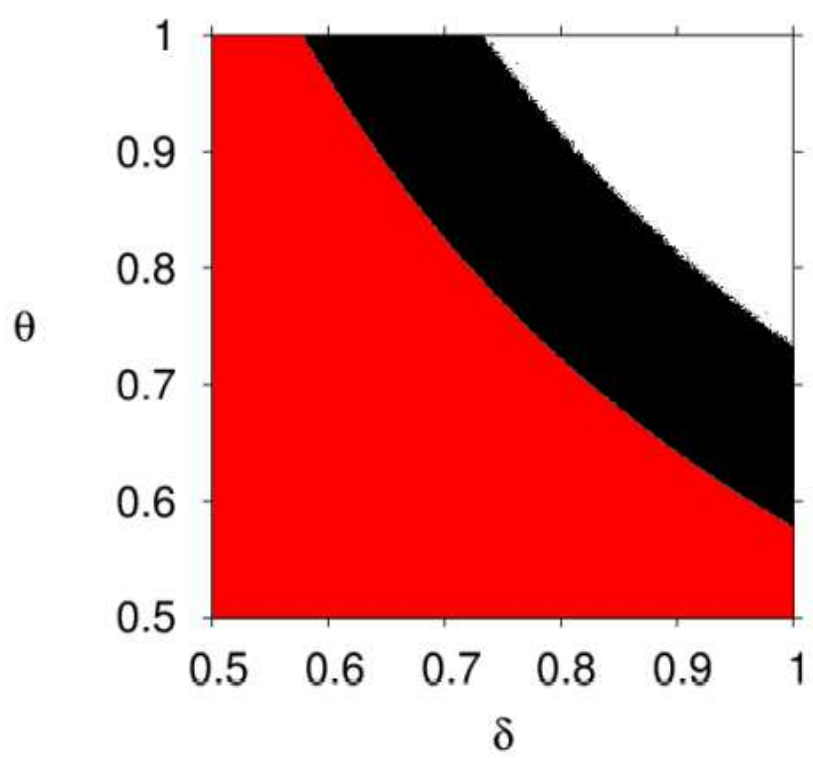

(c) $w_{1}=w_{2}=0.2$

Figure 9

Please see the Manuscript PDF file for the complete figure caption 


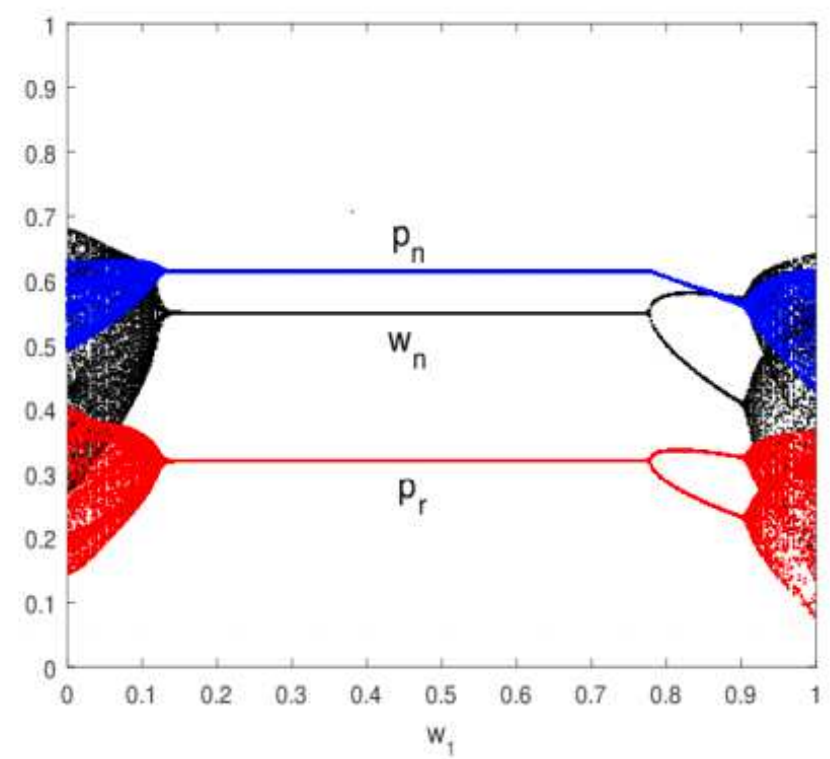

(a)

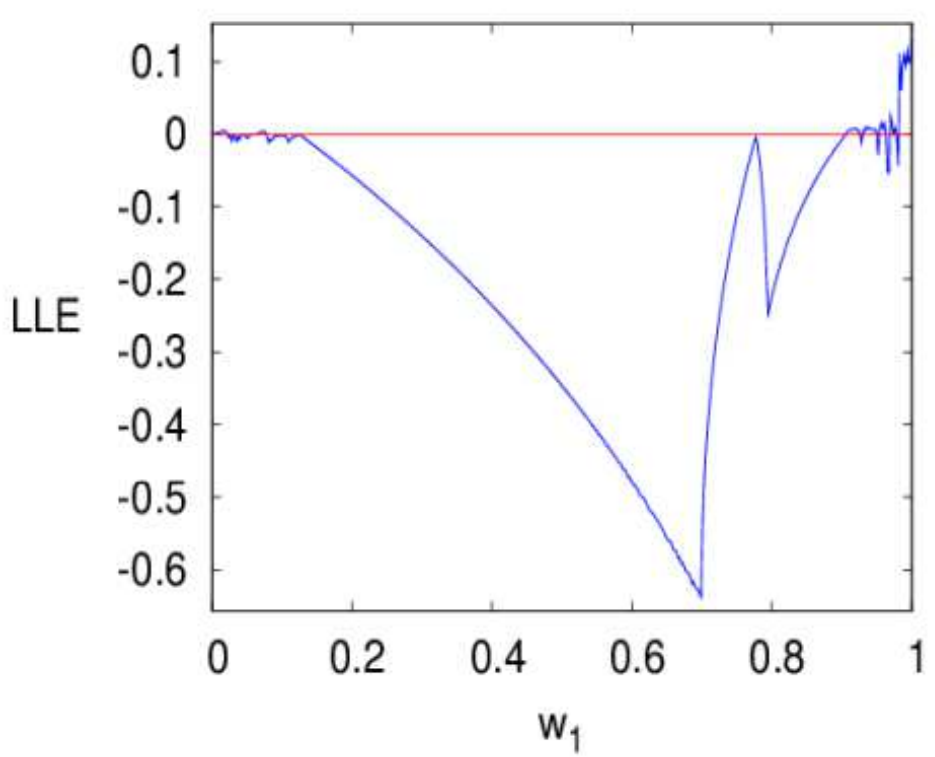

(b)

Figure 10

Please see the Manuscript PDF file for the complete figure caption

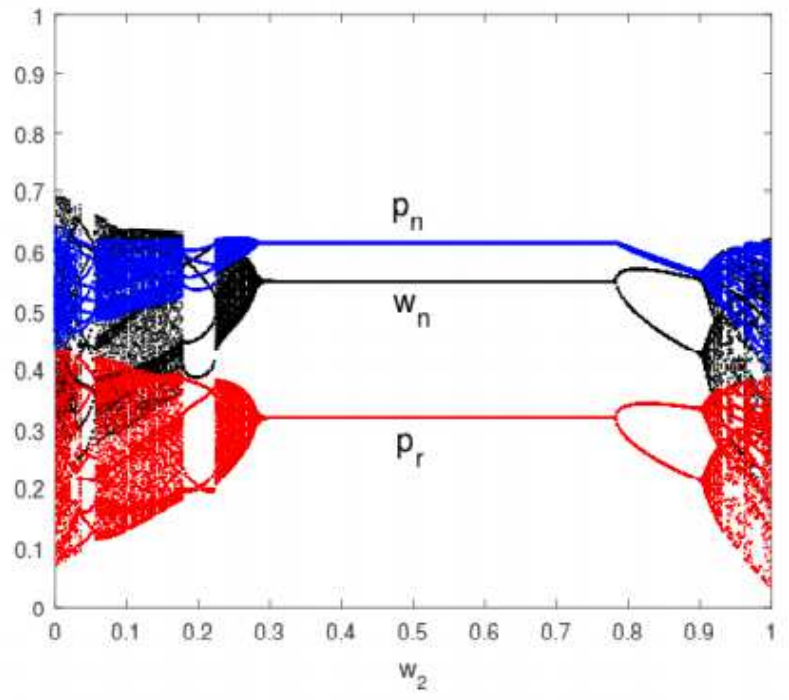

(a)

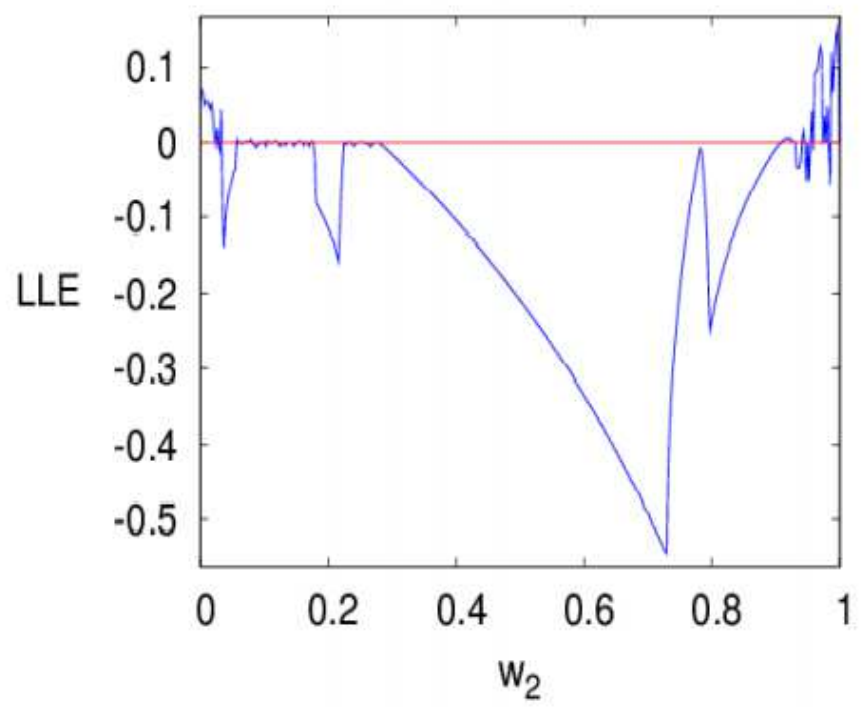

(b)

Figure 11

Please see the Manuscript PDF file for the complete figure caption 


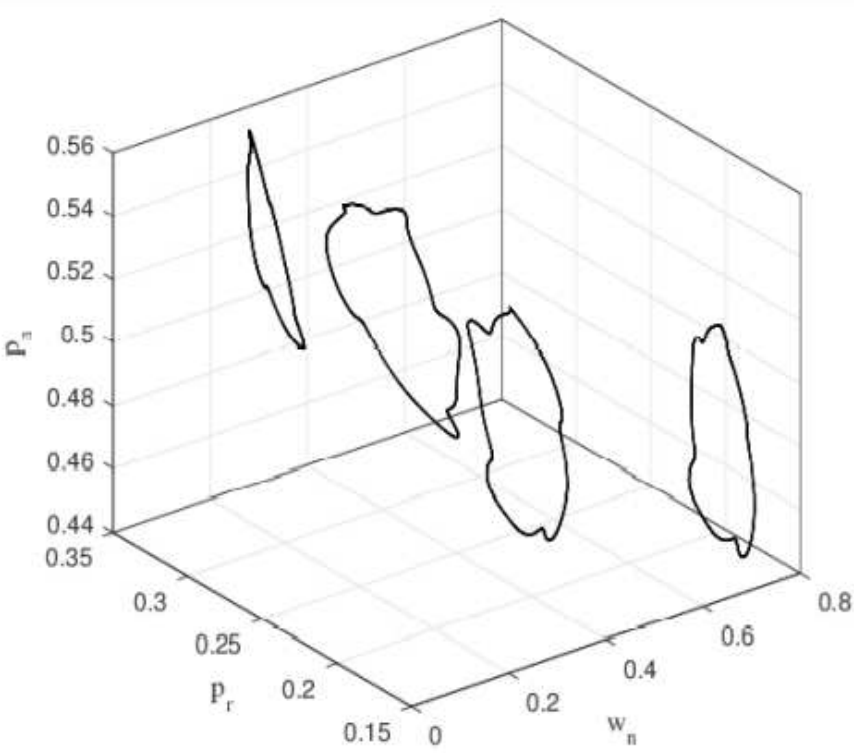

(a) $\alpha=1.45$

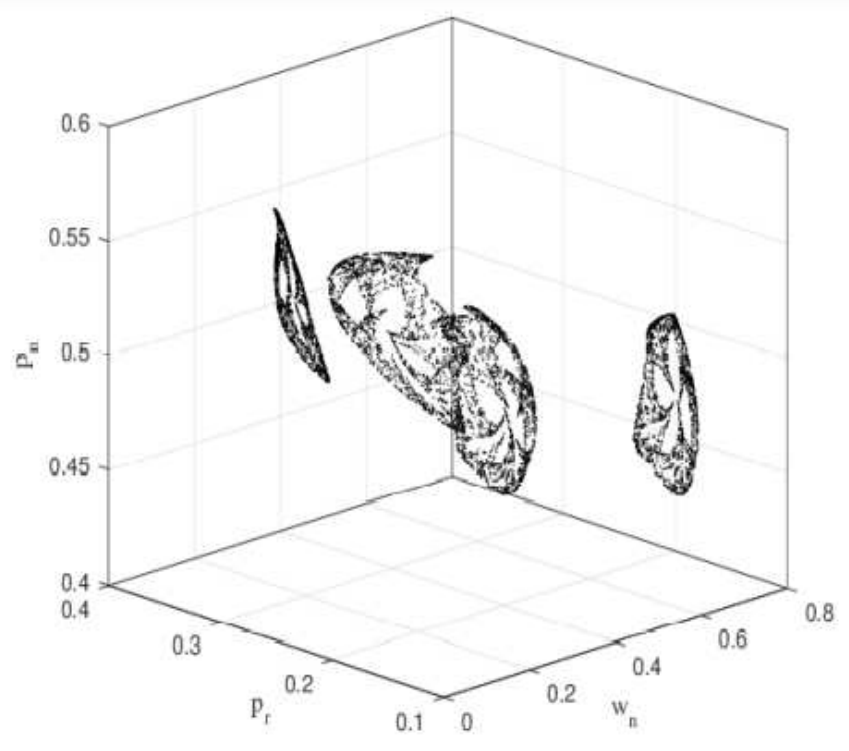

(b) $\alpha=1.48$

\section{Figure 12}

Please see the Manuscript PDF file for the complete figure caption

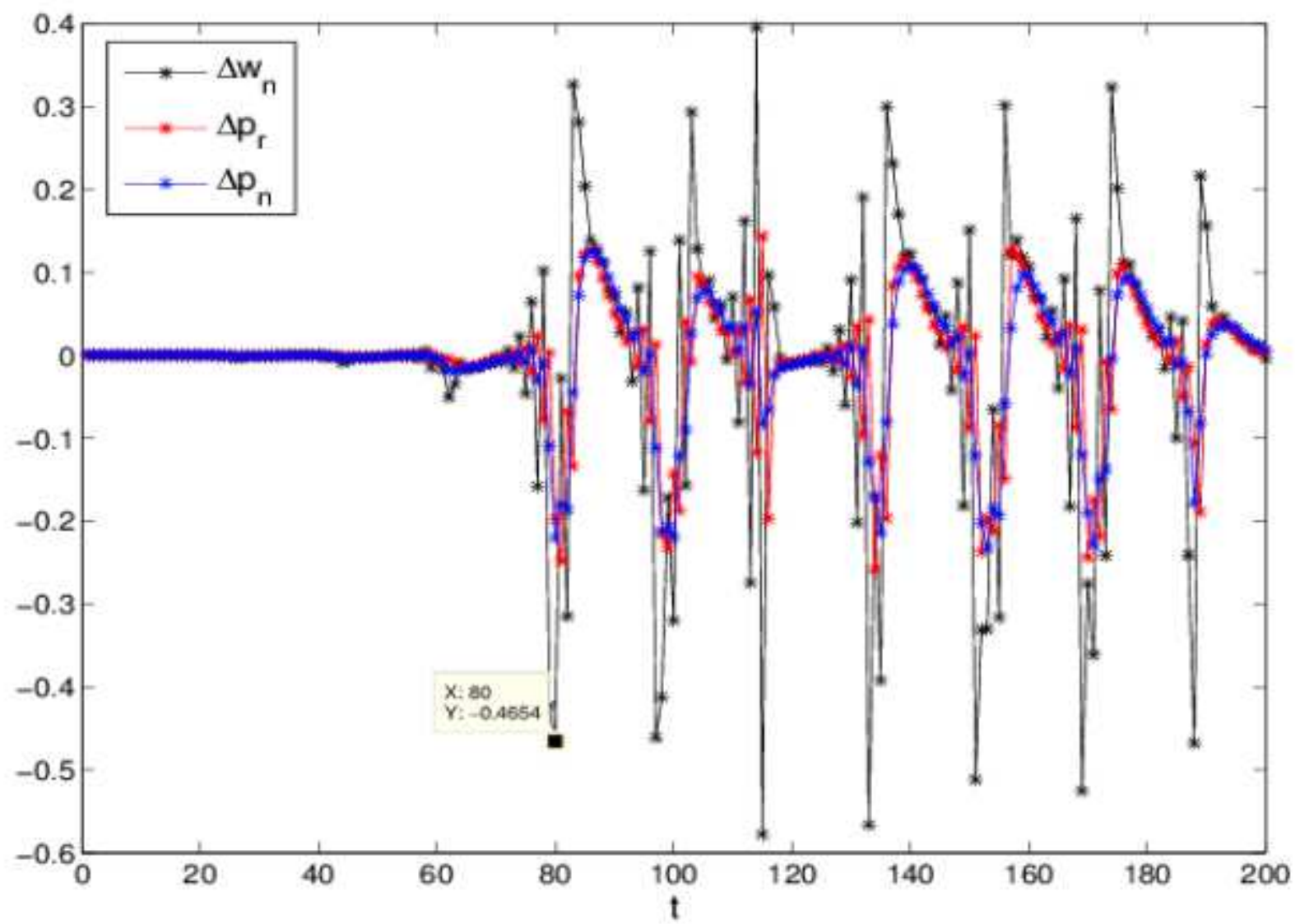

Figure 13 
Please see the Manuscript PDF file for the complete figure caption

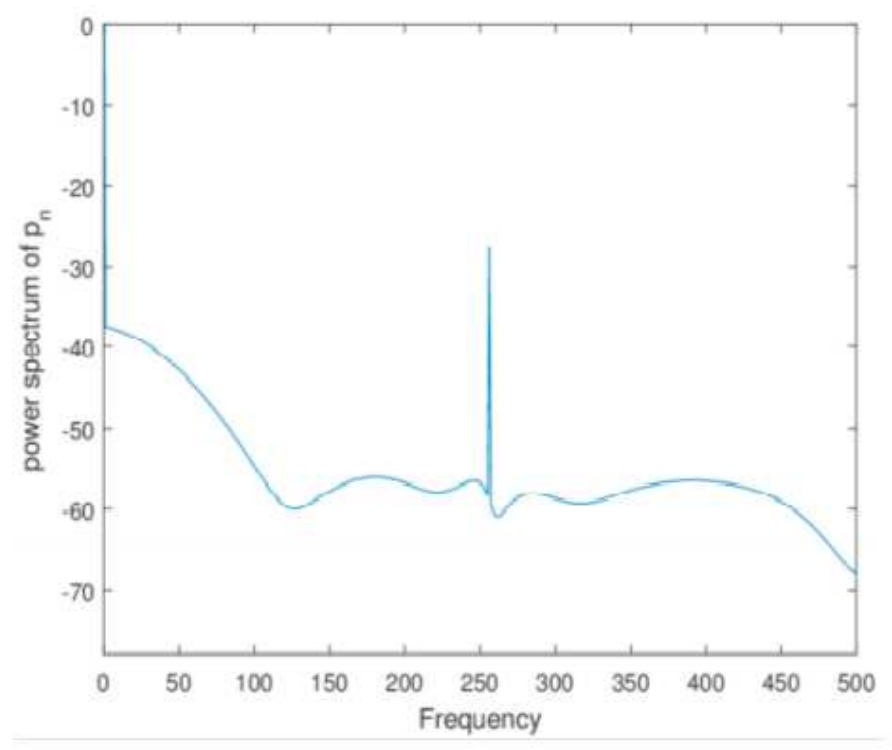

(a) $\beta=1.4($ period-4)

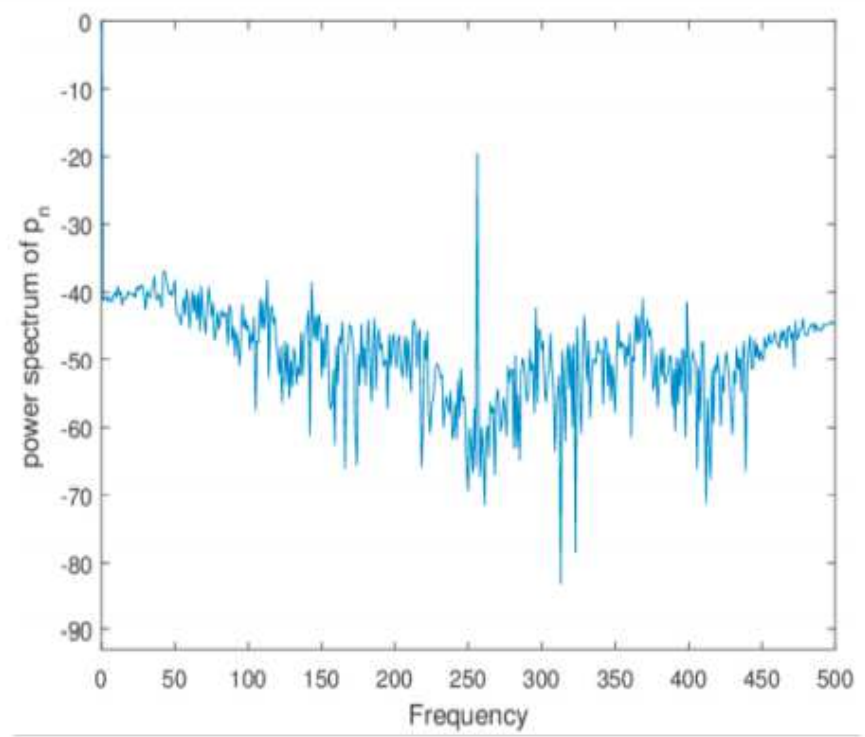

(b) $\beta=1.48$ (chaos)

\section{Figure 14}

Please see the Manuscript PDF file for the complete figure caption

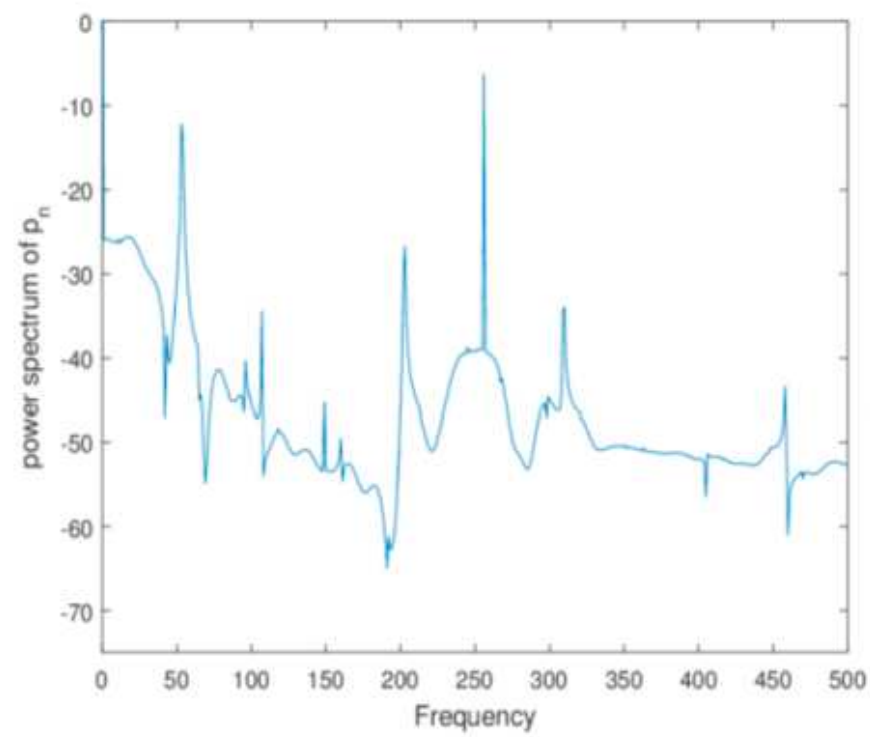

(a) $\alpha=1.44$ (quasiperiodic cycle)

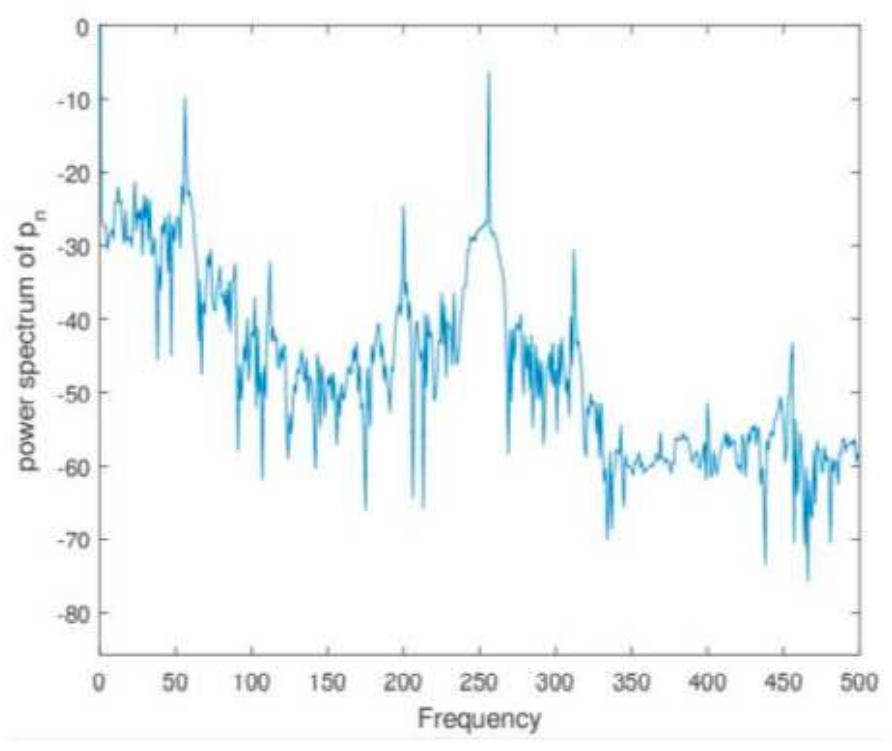

(b) $\alpha=1.48$ (chaos)

\section{Figure 15}

Please see the Manuscript PDF file for the complete figure caption 


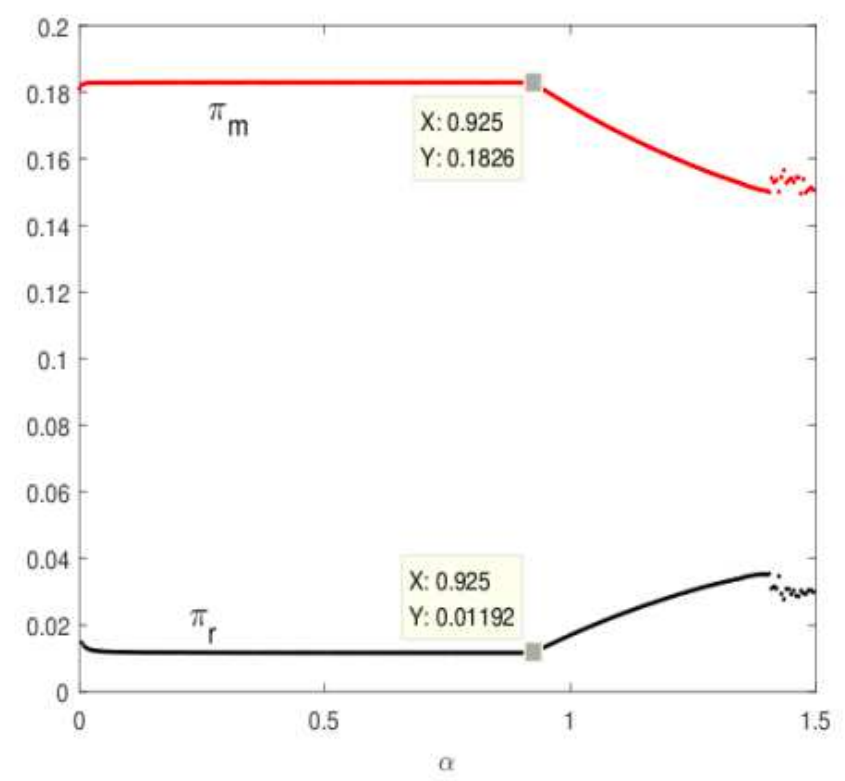

(a) $\beta=v=0.5$

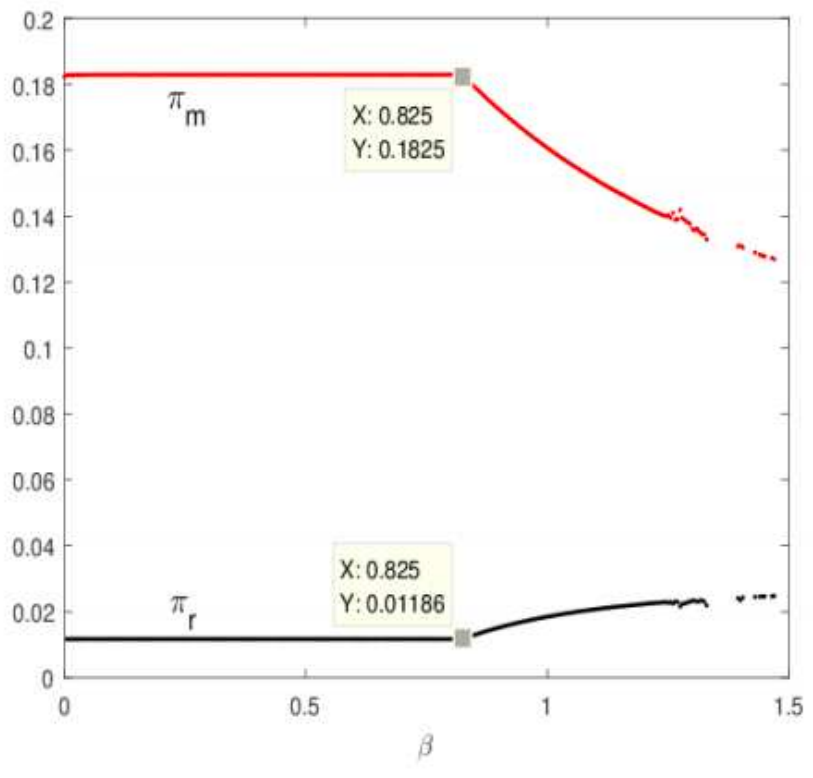

(b) $\alpha=v=0.5$

\section{Figure 16}

Please see the Manuscript PDF file for the complete figure caption

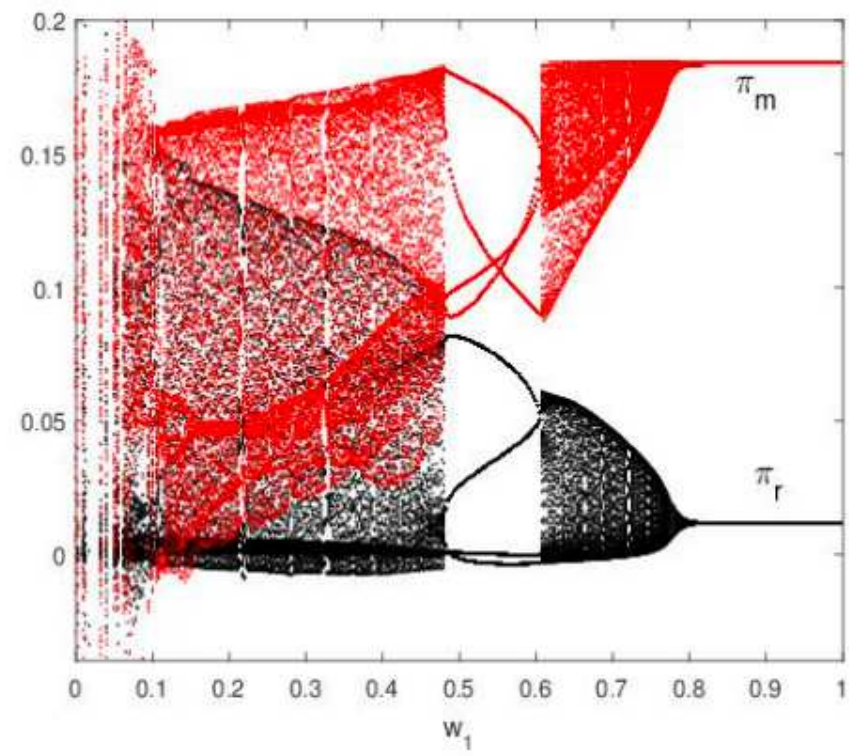

(a)

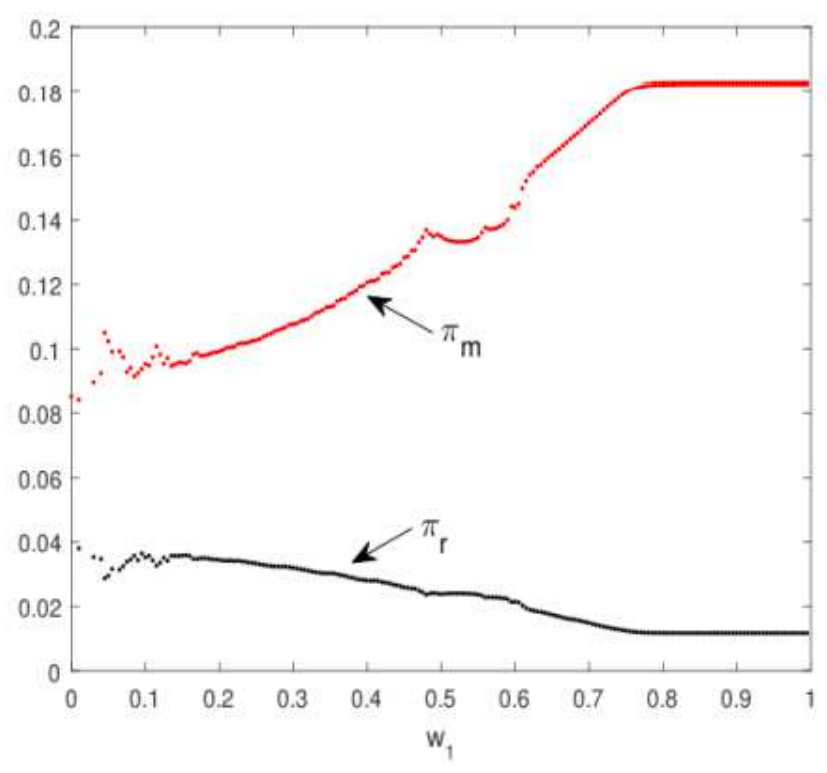

(b)

\section{Figure 17}

Please see the Manuscript PDF file for the complete figure caption 


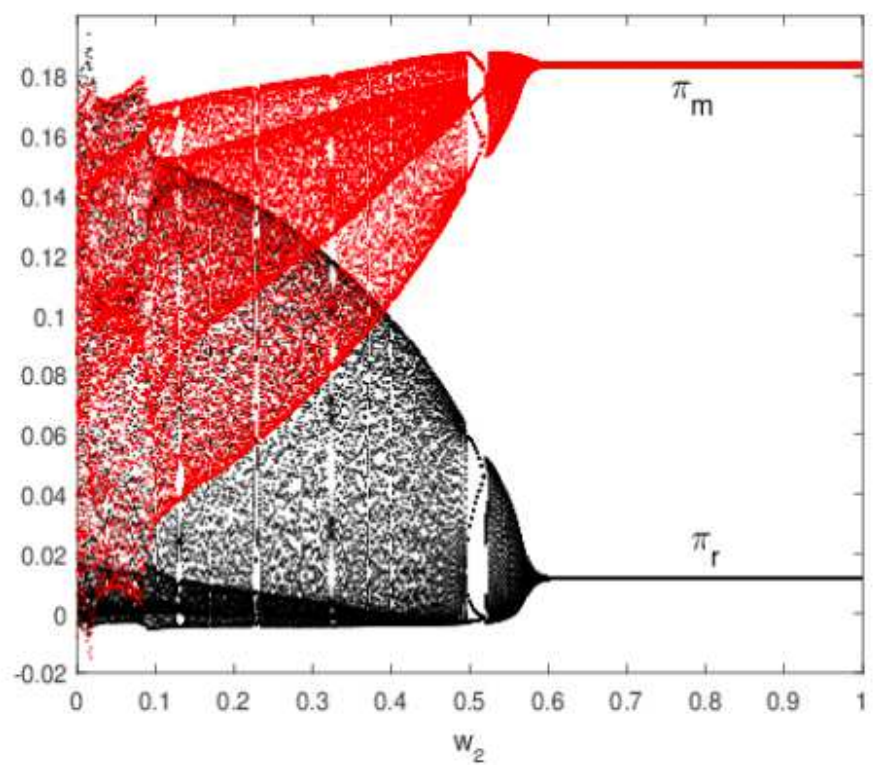

(a)

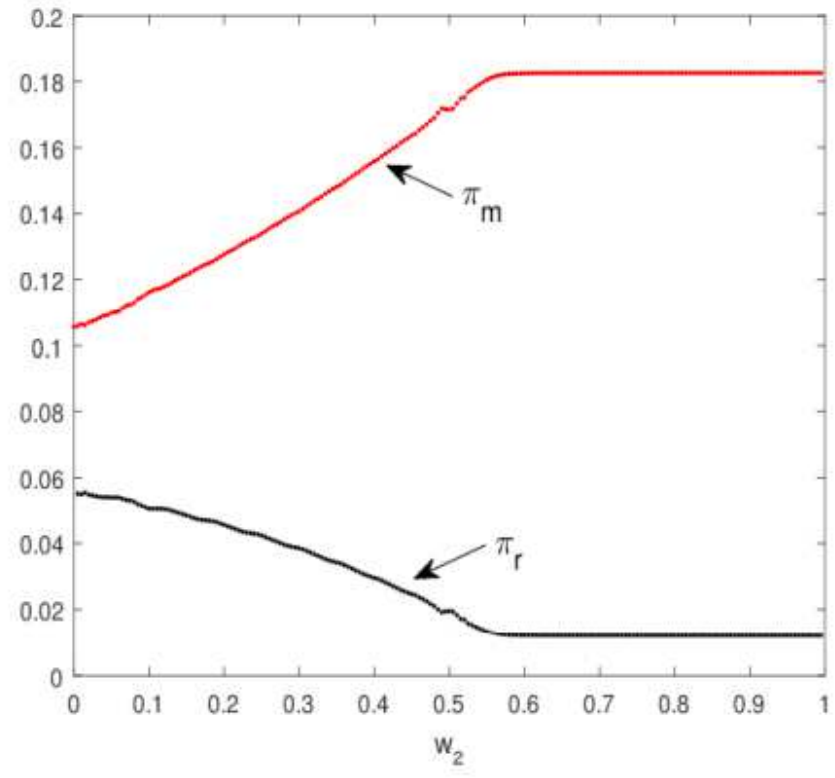

(b)

\section{Figure 18}

Please see the Manuscript PDF file for the complete figure caption

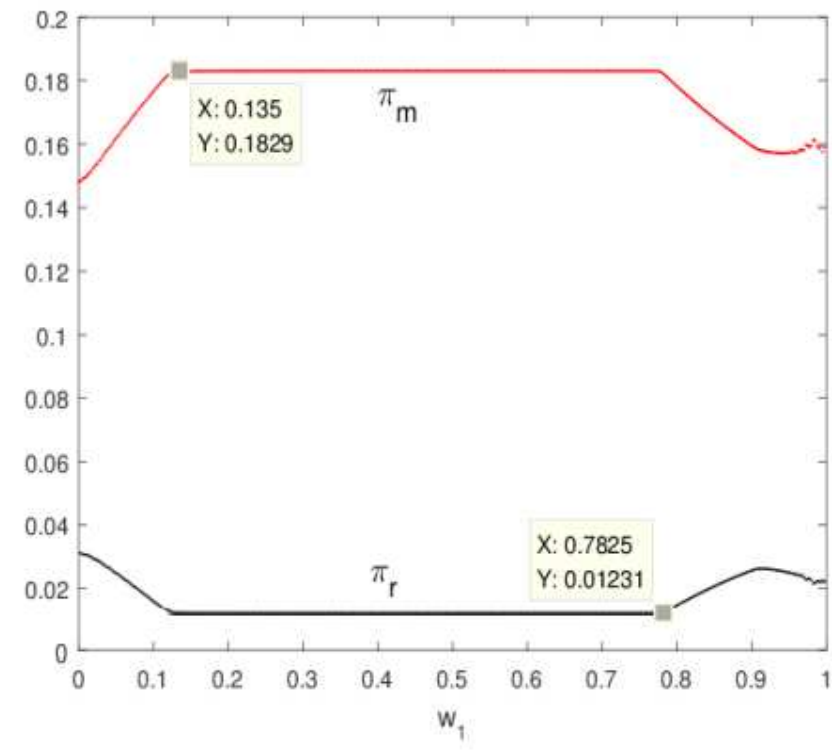

(a) $\alpha=1.1, \beta=0.75, v=0.5, w_{2}=1$

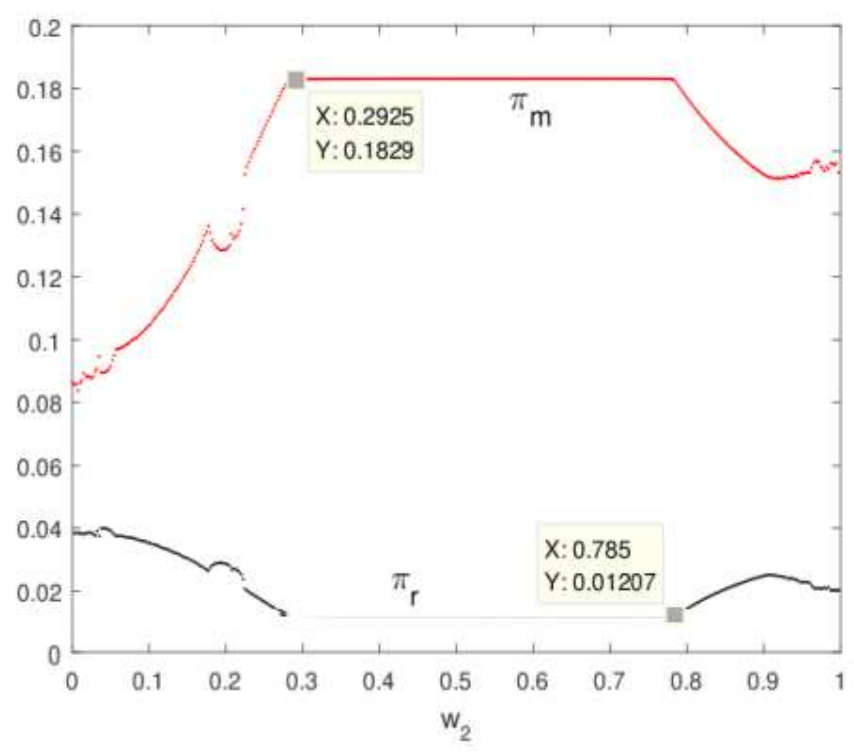

(b) $\alpha=0.85, \beta=1, v=0.5, w_{1}=1$

\section{Figure 19}

Please see the Manuscript PDF file for the complete figure caption 


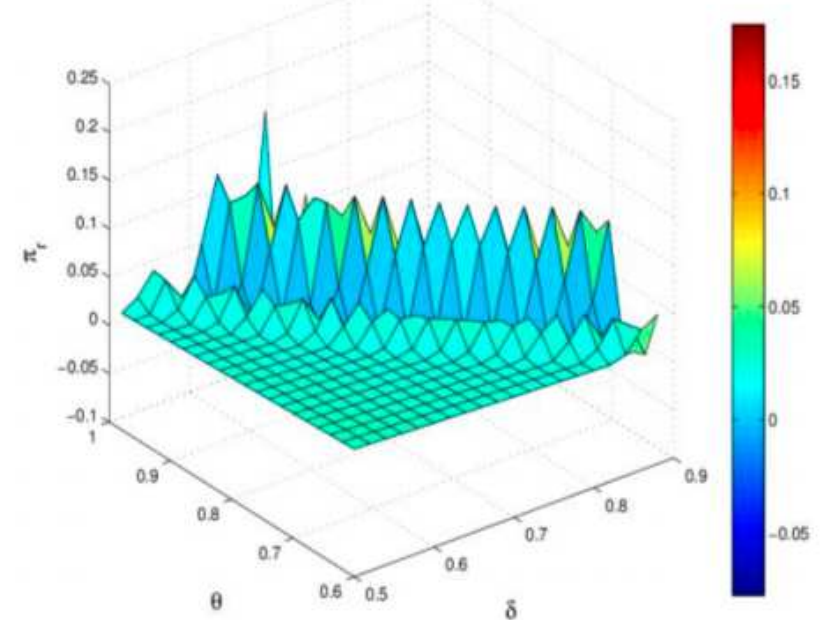

(a)

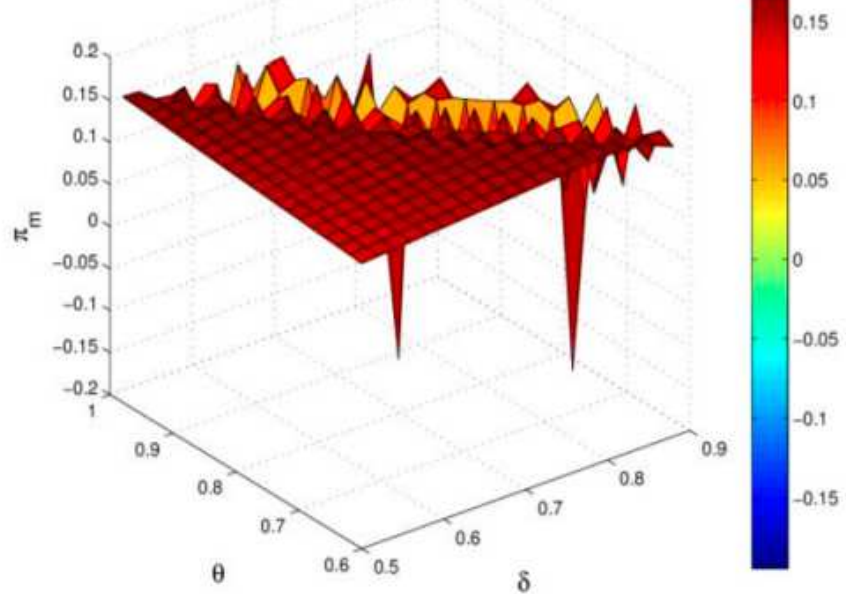

(b)

\section{Figure 20}

Please see the Manuscript PDF file for the complete figure caption

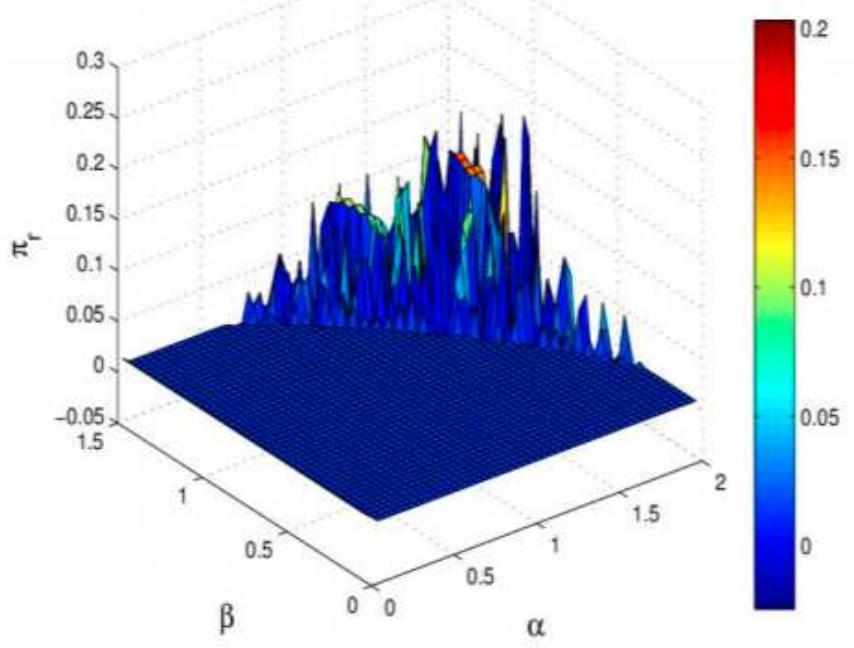

(a)

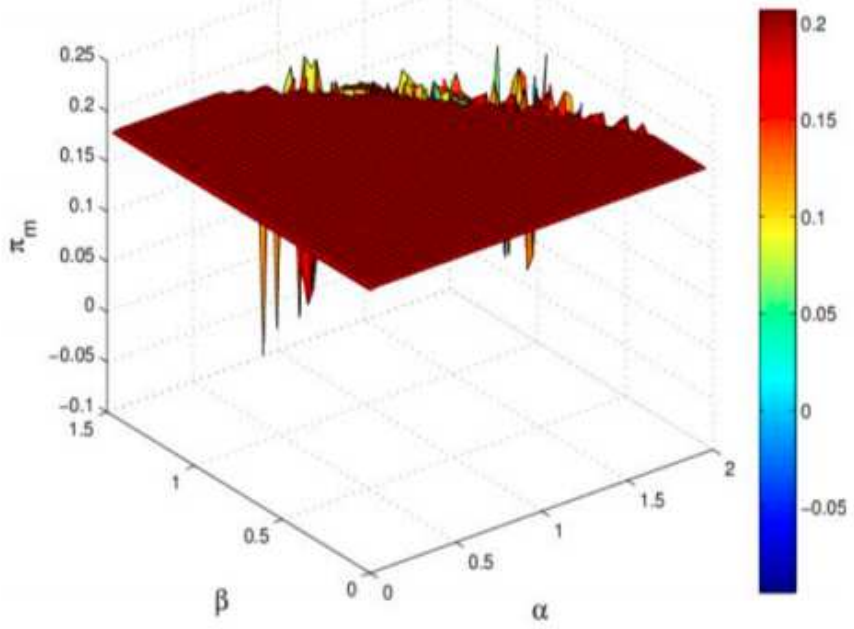

(b)

\section{Figure 21}

Please see the Manuscript PDF file for the complete figure caption 


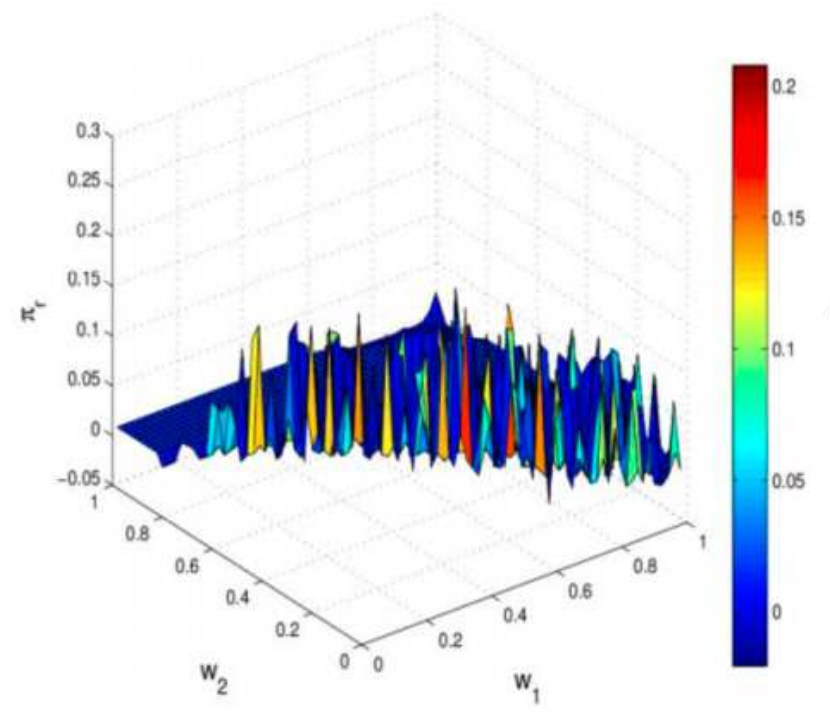

(a)

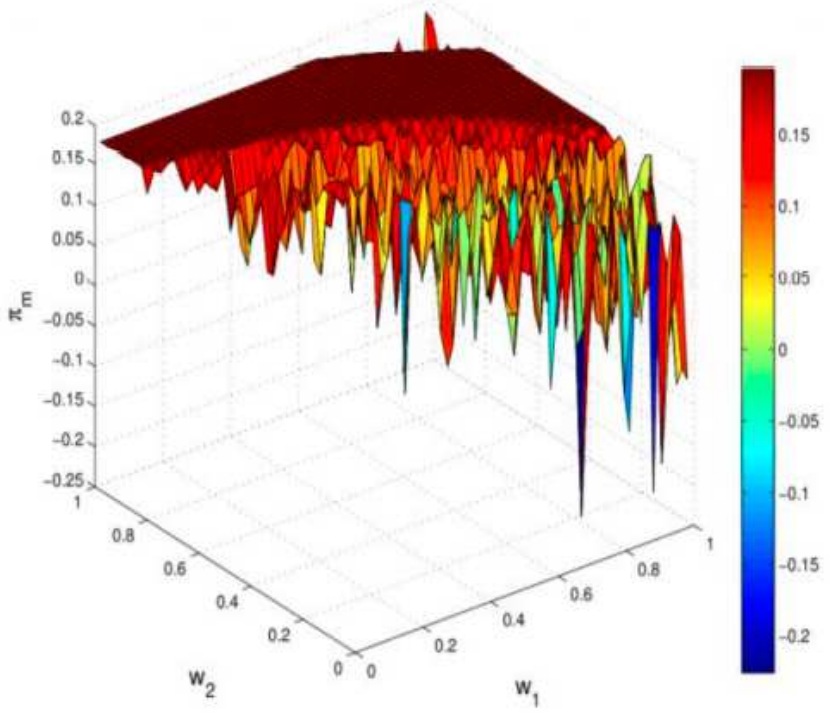

(b)

Figure 22

Please see the Manuscript PDF file for the complete figure caption 


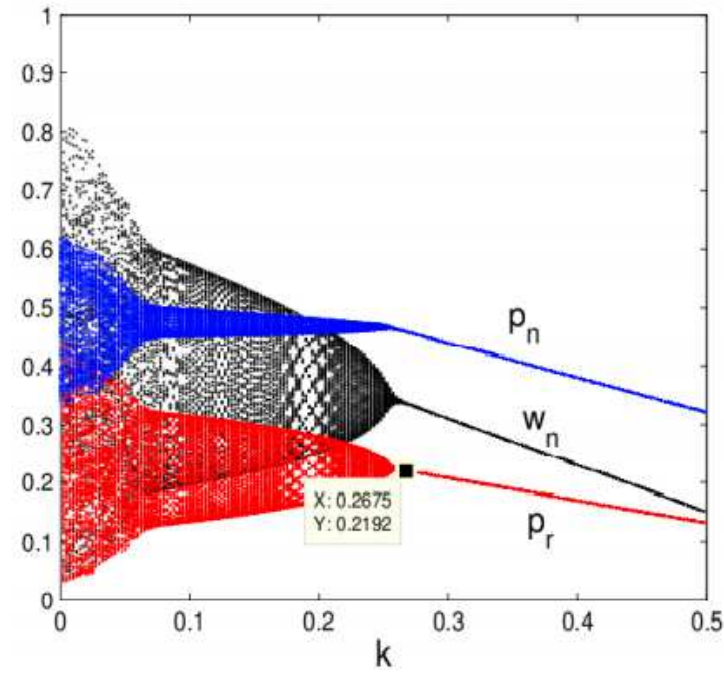

(a)

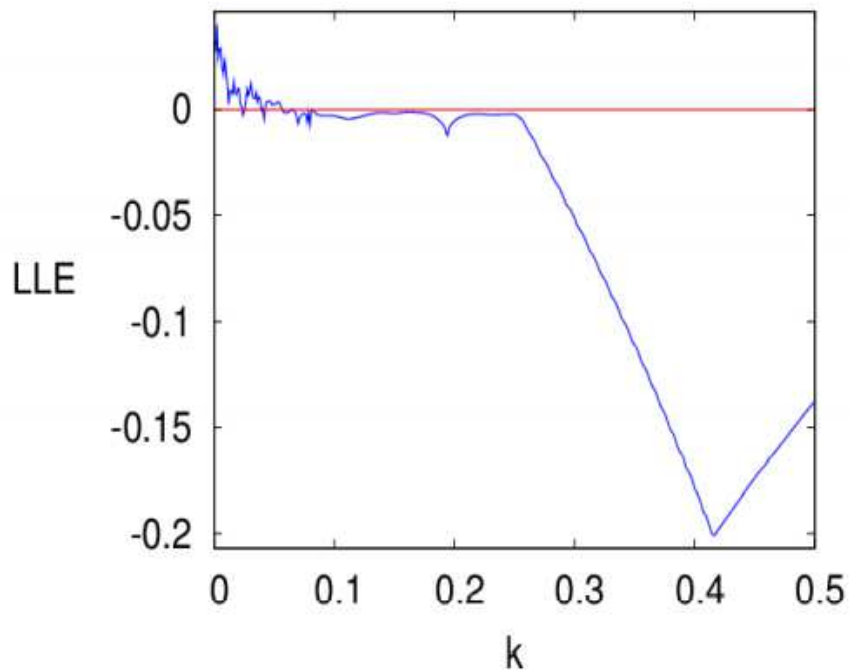

(b)

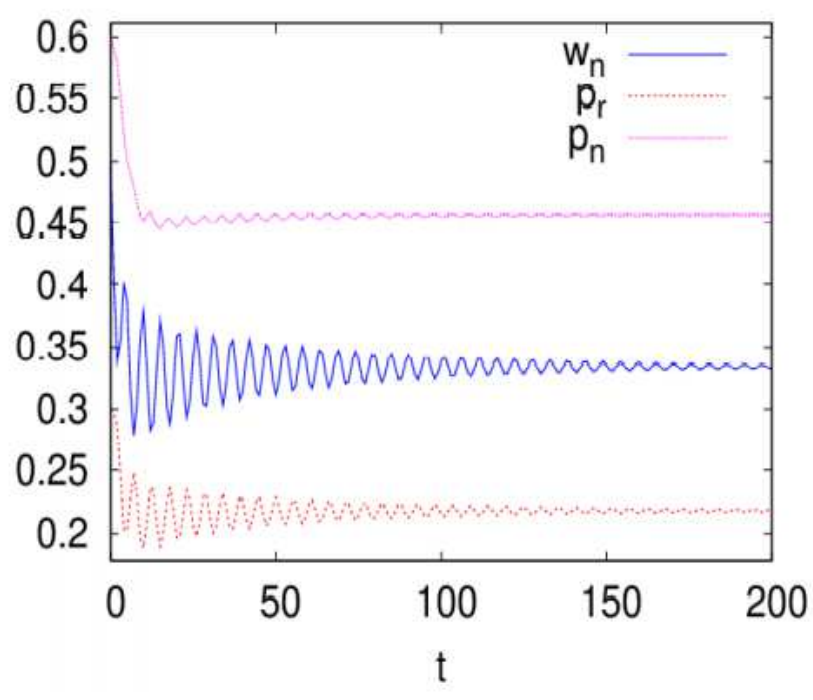

(c) $k=0.27$

\section{Figure 23}

Please see the Manuscript PDF file for the complete figure caption 